\title{
Population Estimates for Phase I \\ Hanford Environmental \\ Dose Reconstruction Project
}

D. M. Beck

A. R. Erickson

S. A. Harkreader

March 1992
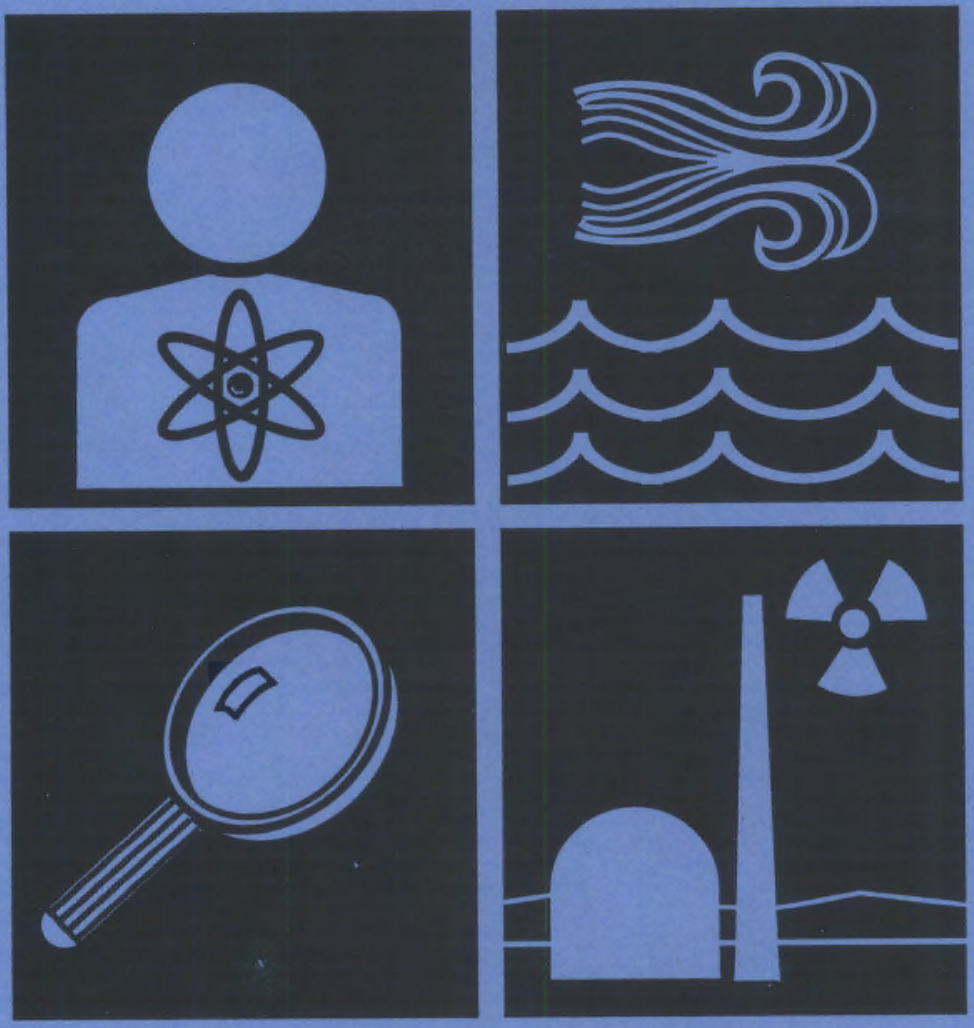

Prepared for the Technical Steering Panel 


\section{DISCLAIMER}

This report was prepared under the direction of the HANFORD ENVIRONMENTAL DOSE RECONSTRUCTION PROJECT Teshnical Steering Panel by Battelle Memorial Institute's Pacific Northwest Liboratories operating the Pacific Northwest Laboratory for the U.S. Department of Energy (DOE). While funding for the work was provided by DOE, the work is not under DOE direction or control. The views and opinions of the authors expressed in this document do not necessarily reflect those of the United States Covernment or any agency thereof. Reference herein to amy specific commercial product, process or service by trade name, trademark, manufacturer or otherwise does not necessarily constitute or imply its endorsement, recommendation or favoring by the U.S. Covernment or any agency thereof, nor by Battelle Memorial Institute.

Printed in the United States of America

Available to DOE and DOE contractors from the

Office of Scientific and Technical Information, P.O. Box 62; Oak Ridge, TN 37831; prices available from (615) 576-8401. FTs 626-8401.

Available to the public from the National Technical Information Service, U.S. Department of Commerce, 5285 Port Royal Rd, Springfield, VA 22161. 
PNL-7263 HEDR

UC -707

POPULATION ESTIMATES FOR PHASE I

Hanford Environmental Dose

Reconstruction Project

D. M. Beck

A. R. Erickson

S. A. Harkreader

March 1992

Prepared for the

Technical Steering Panel

Pacific Northwest Laboratory

Richland, Washington 99352 
POPULATION ESTIMATES FOR PHASE I

March 1992

This document has been reviewed and approved by the Technical Steering Panel.

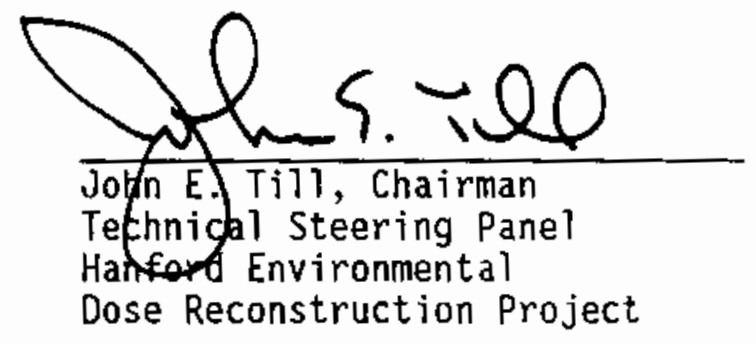

$\frac{31 \text { Jampan } 1992}{\text { Date }}$ 


\section{PREFACE}

This report summarizes the population estimates of Phase 1 of the Hanford Environmental Dose Reconstruction (HEDR) Project. These estimates were used to develop preliminary dose estimates.

\section{PROJECT OBJECTIVES}

Phase $I$ of the HEDR Project had several objectives. Foremost among these was to determine whether sufficient information exists or could be reconstructed from incomplete records to enable a dose reconstruction study to proceed. A second objective was to design conceptual and computational models specifically to deal with uncertainties in the dozens of variables needed to estimate doses to off site populations. The final objectives were to determine whether the data and models were sufficient to enable credible doses to be estimated and to compare HEDR doses with previously published dose estimates. In sumnary, Phase I was a pilot or demonstration phase. The Phase I preliminary dose estimates, which were calculated to demonstrate the feasibility of reconstructing doses, will definitely change as input and model structures are refined in later phases.

It is also important to note that the objectives of the HEDR Project do not include estimating risk or extrapolating to health effects that might have resulted from radiation exposures. A related epidemiological study, the Hanford Thyroid Disease Study, is being conducted for the Centers of Disease Control (CDC) by the Fred Hutchinson Cancer Research Center. This study will seek to determine whether there is increased thyroid disease from exposures to iodine-131 releases during the early years of Hanford Site operation.

\section{OBJECTIVES OF THIS REPORT}

This report was prepared to describe the results of searching for, evaluating, processing, and/or reconstructing the data needed for the feasibility study (Phase I). The report is intended as a demonstration of feasibility and not as a definitive, technical treatise on Hanford data; computer models; or regional demographic, agricultural, or lifestyle patterns. 
Only enough information to demonstrate feasibility was developed, analyzed, and documented in this report.

\section{SUBSEQUENT-YEAR OBJECTIVES}

In the remainder of the project, scientists will identify and minimize errors and shortcomings of Phase I work, including the work reported herein. Scientists will evaluate and enhance the Phase I data and apply improved computer models; evaluate uncertainties in data and models; establish geographic areas, radionuclides, and pathways of interest; support the Hanford Thyroid Disease Study; and carry out activities that will result in better dose estimates for specified populations and for individuals.

The term "Phase $I "$ is used in this report to refer to the first of four phases originally planned for the project. Phase I ended in July 1990. In February 199I, the TSP decided to shift the project planning approach away from phases--which were centered around completion of major portions of technical activities--to individual fiscal years, which span october of one year through September of the next. Therefore, activities that were previously planned for one of the three remaining phases are now designated to occur in one or more of the next several fiscal years.

\section{FUTURE DOCUMENTATION}

Much of the HEDR documentation is yet to be produced. The scope, level of detail, level of peer review, and mode of publication for each document will be defined during task planning. It is expected that documents will fall into one of four categories:

- summary documents intended primarily for the non-technical lay reader

- summary documents intended primarily for the general technical reader

- detailed topical documents intended primarily for the technical peer reviewer (e.g., refereed technical journals)

- letter reports to transfer information among HEDR tasks or related projects or to provide information to the TSP or its subcommittees. 
The intention is to provide information for readers with all levels of interest.

\section{PROJECT DIRECTION, QUALITY ASSURANCE, AND COMMENT INCORPORATION}

The HEDR Project is directed by an independent Technical Steering Panel (TSP) of scientists and representatives of the states of Oregon and Washington, of regional Native American Tribes, and of the public. The TSP's charter is to direct, review, evaluate, and approve all HEDR Project work. The work described here was conducted by Battelle staff at the Pacific Northwest Laboratory. The U.S. Department of Energy funded Phase $I$ of the project, but provided no technical direction, oversight, or review. Beginning in mid-1992, Battelle will continue dose reconstruction activities under contract to the Centers for Disease Control, which will fund HEOR and TSP work. The TSP will continue to provide technical direction for the project.

The work described in this report was conducted in accordance with the requirements of ANSI/ASME NQA-1 1986 Edition, Quality Assurance Program Requirements for Nuclear Facilities, as interpreted by the PNL Quality Assurance (OA) program.

Appendix $F$ is a record of ISP comments and Battelle's responses to those comments; the TSP has reviewed and approved Battelle's responses. The comment numbers appear in this document in the left margin next to the paragraphs in which the corresponding comments are addressed. Any text that has been changed is shown in italics. In addition to changes to address TSP comments, some text has been changed for correction of errors and for further clarification. 
، 


\section{SUMMARY}

The Hanford Environmental Dose Reconstruction (HEDR) Project was estabJished in 1987 to estimate radiation doses that people could have received from nuclear operations at the Hanford Site since 1944. To achieve this objective, demographic information must be developed that describes the study population in enough detail to allow researchers to identify potentiaily exposed groups of people and the number of people in each of those groups. The Phase I study area consists of the ten-county area around the Hanford Site. The required demographic information for Phase I includes year-by-year estimates of county and subdivision populations by age, sex, and race.

Three methods were used to develop county population estimates: ratio correlation, component method II, and vital rates. When results using the three methods were compared with a special census of Benton County in 1944, the ratio-correlation estimates were far superior (3.3\% deviation from actual census count with a standard addition for transient construction workers). The superiority of this method was also evident in comparisons with actual census data from 1950, 1960, and 1970. The ratio correiation estimates were, therefore, used as the control totals for developing intercensal age, sex, and race estimates for counties and county subdivisions.

A cohort-component model developed by Dr. Donald Pittenger for estimating intercensal county populations by age and sex was not run to produce Phase I estimates because of lack of data and time constraints. In addition, the level of additional accuracy potentially gained by using this model was not considered necessary for Phase I. Instead, Dr. Pittenger used a combination of cohort interpolation and age-group interpolation estimates to provide these county population estimates by age and sex (Pittenger 1991). Race-share estimates were interpolated. Intercensus population estimates for all county census divisions within the HEDR study area were then calculated for the years 1940 to 1970 by interpolating population shares between the two census counts bracketing a particutar estimate year. 


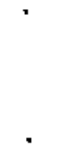




\section{CONTENTS}

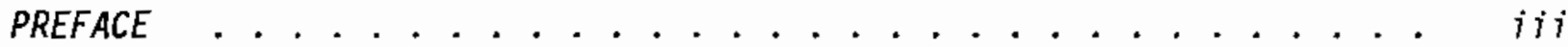

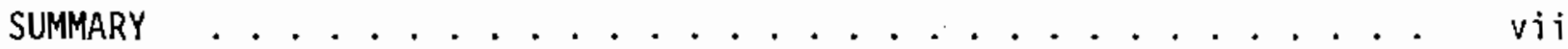

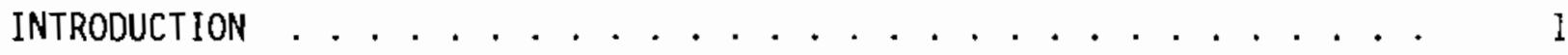

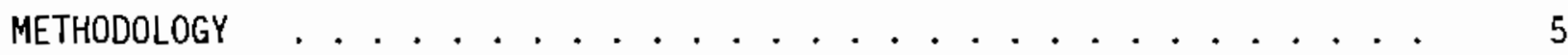

STEP 1: DEVELOPING COUNTY POPULATION ESTIMATES . . . . . . . 5

Information Sources for HEDR Population Estimation . . . . 7

Ratio-Correlation Population Estimation Method ...... 9

Component Method II Population Estimation Method . . . . . 12

Vital Rates Population Estimation Method ........ 13

Selection of Estimates for Use in Subsequent Steps . . . . 14

STEP 2: DEVELOPing COUNTY-LEVEL AGE, SEX, and RACE ESTIMATES . . 17

Age and Sex Estimation Methods ........... 17

Race Estimation Method .............. . . 18

STEP 3: DEVELOPING POPULATION ESTIMATES FOR

COUNTY CENSUS DIVISIONS .......................... 19

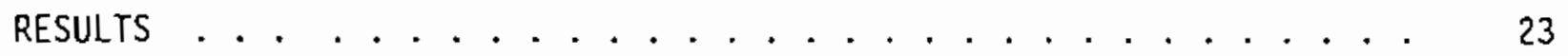

COUNTY-LEVEL HEDR ESTIMATES 1940 THROUGH $1970 \ldots \ldots . \ldots . \ldots 23$

SUBCOUNTY HEDR POPULATION ESTIMATES . . . . . . . . . . 49

REFERENCES ................... . . . 59

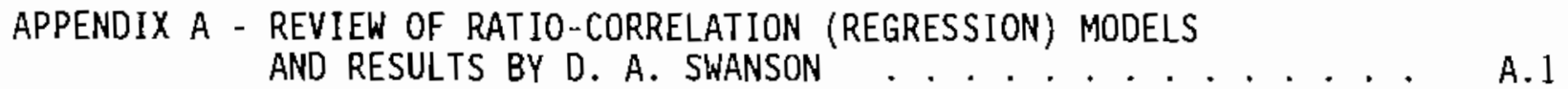

APPENDIX B - REVIEW OF HEDR MODELS AND RESULTS BY D. B. PITTENGER . . B.I

APPENDIX $\subset$ - STEPS IN DEVELOPING RATIO-CORRELATION ESTIMATES . . . . c. c.

APPENDIX D - USE OF COMPONENT METHOD II PROCEDURE TO ESTIMATE

1947 YAKIMA COUNTY POPULATION . . . . . . . . . . . 0.1 
APPENDIX E - ESTIMATING COUNTY CENSUS DIVISION POPULATIONS $\ldots \ldots$ E.1 APPENDIX F - SUMMARY OF TSP COMMENTS AND BATTELLE RESPONSES . . . . . F.I 


\section{FIGURES}

1 Hanford Environmental Dose Reconstruction

Study Area for Phase I Research . . . . . . . . . . . . 2

2 Overview of Methodology Used to Develop HEDR Phase I

Population Estimates . . . . . . . . . . . . . 6

3 Hanford Environmental Dose Reconstruction

County Census Divisions ............... 20

4 Adams County Population Estimates 1940 through 1970 . . . . . . 24

5 Benton County Population Estimates 1940 through 1970 . . . . . . 24

6 Frankl in County Population Estimates 1940 through 1970 . . . . . 25

7 Grant County Population Estimates 1940 through 1970 . . . . . 25

8 Kittitas County Population Estimates 1940 through 1970 . . . . 26

9 K7ickitat County Population Estimates 1940 through 1970 . . . . 26

10 Walla Walla County Population Estimates 1940 through $1970 \ldots 27$

II Yakima County Popuiation Estimates 1940 through $1970 \ldots 27$

12 Morrow County Population Estimates 1940 through $1970 \ldots 28$

13 Umatilia County Population Estimates 1940 through $1970 \ldots 28$ 


\section{IABLES}

1 Ratio-Correlation Model (OLS) for Washington

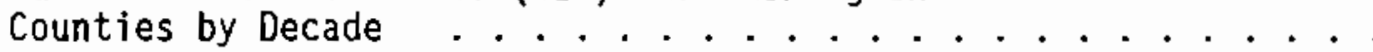

2 Comparison of 1944 Benton County Population Estimates and 1944 Benton County Special Census Population Total . . . . . . 16

3 Deviation of Census Year Estimates from Actual Census Counts . . 16

4a Population Estimates for Washington Counties 1941 - $1946 \ldots 29$

4b Population Estimates for Washington Counties 1947 - 1953 . . . 33

4c Population Estimates for Washington Counties 1954 - 1960 . . . 37

4d Population Estimates for Washington Counties 1961 - 1967 . . . 41

4e Population Estimates for Washington Counties 1968 - 1970 . . . 45

5a Adams County Population by Division . . . . . . . . . . . 49

5 b Benton County Population by Division ........... 50

$5 c$ Franklin County Population by Division .......... 51

5d Grant County Population by Division . . . . . . . . . . 52

5e Kittitas County Population by Division ........... 53

$5 f \mathrm{Klickitat}$ County Population by Division . . . . . . . . 54

$5 g$ Walla Walla County Population by Division .......... 55

5h Yakima County Population by Division .......... 56

$5 i$ Morrow County Population by Division . . . . . . . . . . 57

$5 j$ Umatilla County Population by Division ........... 58 


\section{INTRODUCTION}

The objective of the Hanford Environmental Dose Reconstruction (HEDR) Project is to develop estimates of the radiation doses populations could have received from Hanford operations beginning in 1944. To achieve this objective, demographic, lifestyle, agricultural, and food consumption information will be needed. Demographic information must be developed that describes the study population in enough detail to allow researchers to identify potential exposure groups and to estimate the number of people in each of those groups. Not all the demographic needs of the HEDR Project can be identified at this phase of the project; however, existing dose models suggest that, at a minimum, information on the age, sex, and race composition of the study population will be needed, as well as information on population density, migration patterns, and residential location. Other demographic parameters, such as income level and occupation, may also be important because they are closely associated with lifestyle and food consumption.

Requirements for providing the detailed demographic information outlined above include accurate estimates of county populations and information on where within a county people resided. This information is essential because these factors influence the type of food people eat and whether or not their food is produced locally. This is particularly true for the earlier years of the study period. For example, if 1945 data for a particular county indicate that the population was widely scattered throughout the county with few incorporated areas, it is reasonable to assume that many of the residents were more self-sufficient in terms of food and milk production than their urban counterparts. As a result, the rural residents of the county were likely to have consumed homegrown vegetables and milk from backyard cows, behavior that may have increased their exposure to certain radioisotopes. The point here is that demographic information is crucial to a description of settlement patterns and lifestyles which, in turn, are critical for determining exposure rates for the different population groups living in the Phase I study area.

The Phase I study area consists of eight counties in central Washington and two counties in northern oregon (Figure 1). The purpose of this report is 


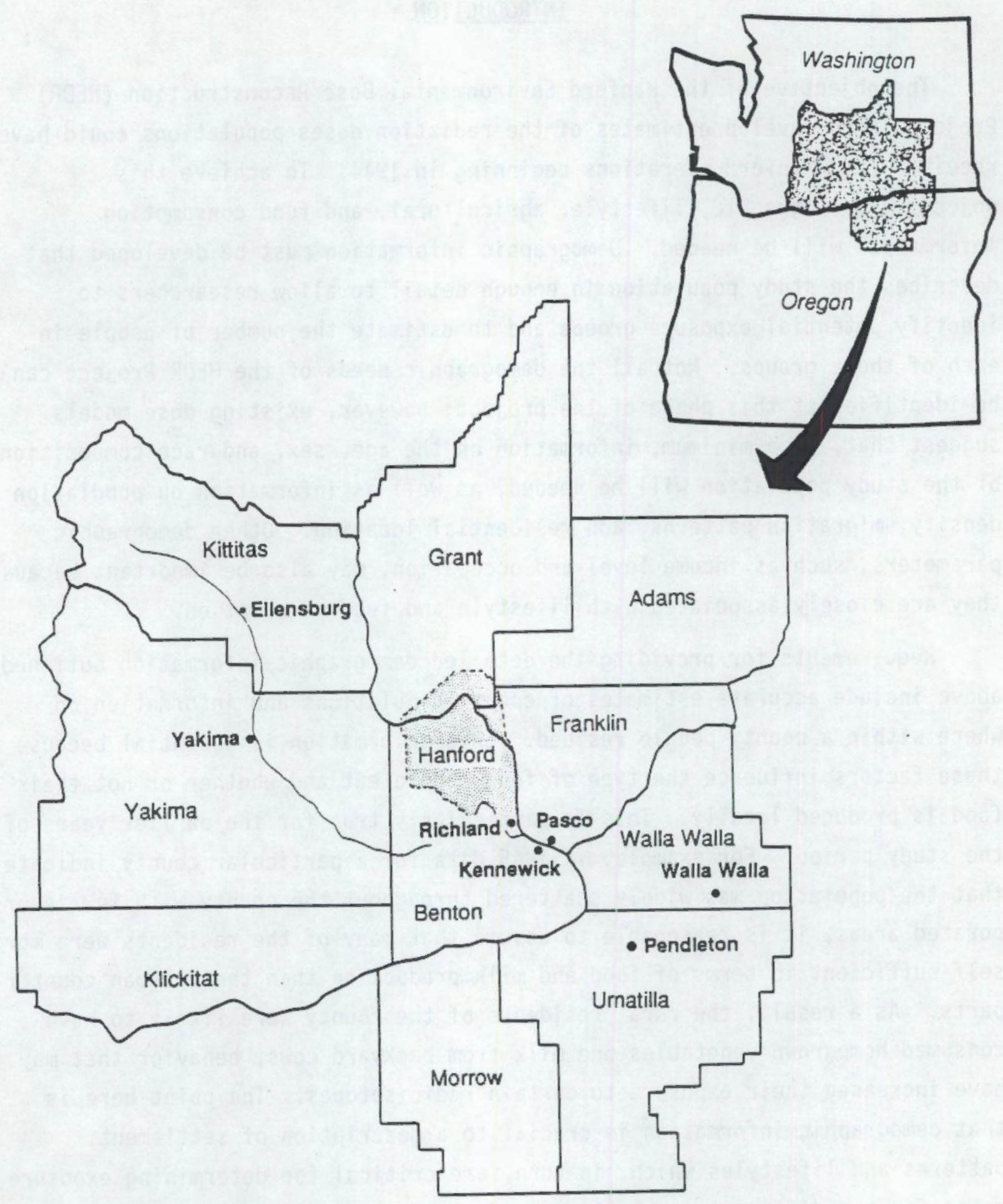

FIGURE 1. Hanford Environmental Dose Reconstruction Study Area for Phase I Research 
to report initial Phase I population estimates and to explain how they were developed. Three general steps were used to develop the Phase I estimates: 1) county population estimates were developed; 2) age, sex, and race details were developed for each county; and finally 3) populations for sub-county areas were developed by disaggregating county totals to county census divisions (CCDS). Sections of this report discuss the methods associated with each of the three steps and the resuits of county and subcounty population estimates. Appendices contain evaluations of the estimates by outside reviewers and detailed descriptions of certain sub-steps. Age/sex/race component estimates are contained in a separate HEDR report (Pittenger 1991).

\section{Quality Assurance}

The work described in this report was conducted in accordance with the requirements of ANSI/ASME NQA-1 1986 Edition, Qual ity Assurance Program Requirements for Nuclear Facilities, as interpreted by PNL's Quality Assurance (QA) program.

All staff performing work described here are adequately trained and qualified. All procedures used to support this report were appropriately written and controlled in accordance with PNL QA program requirements. Records that support the data in this report were created and stored in accordance with applicable HEDR record control requirements.

Results were reviewed by a $Q A$ representative to ensure that the results were traceable back to the raw data and that the work was performed in accordance with applicable QA requirements. The staff member entering the data into databases ensured correct entry by comparing raw data with the hard copy of the data listing and indicated acceptance by signing and dating the hard copy. Calculations used to produce the reported results were checked as a primary verification of accuracy and correctness. The checking was performed by qualified staff members who did not perform the calculations. Through this informal $Q A$ check, it was determined that adequate data traceability existed.

The ratio-correlation model and the county population estimates produced with it were submitted to Dr. David Swanson, a demographer specializing in 
ratio-correlation estimates. He concluded that the model and corresponding estimates are accurate. A complete copy of his review is found in Appendix A.

All three models for estimating intercensal county population were submitted to $\mathrm{Or}$. Donald Pittenger, along with the estimates produced by these modeis. Or. Pittenger is director of the Demographics Laboratory in 0iympia, Washington and has extensive experience in preparing migration estimates for the state of Washington. He served as staff demographer for the Washington State office of Financial Management, where he was responsible for developing the official state population estimates. Although he raised several minor issues, he concluded that the models produce accurate results. A complete copy of his review is contained in Appendix $B$.

Possible Steps to Refine Estimates

By combining the results from the methods discussed in this paper, accurate population estimates for the HEDR Phase I counties and CCDs have been developed. However, further refinement is always possible. Perhaps the most cost-effective refinement would be to run the cohort component II models developed by Dr. Pittenger but not used due to lack of data and time constraints. Another possible refinement would involve using aerial photographs available for the CCDs adjacent to the Hanford size to produce population estimates based on the housing unit procedure. However, as with all demographic estimates, the decision to refine estimates must balance the expected increase in accuracy against the level of activity associated with the methods. We expect to use the results from future sensitivity analyses to help determine the appropriate level of future effort this task warrants. 


\section{METHODOLOGY}

To identify potential exposure groups within the HEDR Phase I study area and to estimate the number of people in each of those groups, researchers need subcounty population estimates by age, sex, and race for each year from 1944 to 1947 and 1964 to 1966. Estimates were prepared for 1940 to 1970 to ensure that estimates that may be needed for future HEDR work will be available. The following sections describe the steps and associated methods used to produce these estimates: 1) developing county population estimates, 2) developing county-level age, sex, and race component estimates, and 3) developing county census division estimates. An overview of the methodology is provided in Figure 2 .

\section{STEP 1: DEVELOPING COUNTY POPULATION ESTIMATES}

The first step in reconstructing the demographic history of the HEDR study area is to prepare county population estimates. In the U.S., complete censuses are routinely conducted only the first year of every decade. Therefore, to map population change throughout a decade, inter-census estimates must be developed. Over the last several decades several estimation methods have evolved, and two (the component method II and ratio-correlation methods) have become the accepted standards in the demography discipline. Both of these methods were used for this project and are discussed later in this report.

All of the estimation methods address in one way or another the components of the most basic equation in demography, the balancing equation. This equation decomposes population change into its components and can be expressed as follows:

$$
\text { Pt }-P_{0}=B-0+I-0
$$

where $P t=$ the population at the end of the period

Po = the population at the beginning of the period

$$
B=\text { births }
$$




\section{STEP 1: Develop County Population Estimates:}

- Produce estimates for each year 1940 to 1970 - using several methods:

- ratio-correlation method

- component method II

- vital rates method.

- Adjust Benton County estimates for influx of construction workers.

- Compare estimates with available census counts.

- Select set of estimates for use in subsequent steps.

\section{STEP 2: Develop County-Level Age, Sex, and Race Estimates}

- Produce age/sex share estimates for each year 1940 to 1970 using several methods:

- cohort component model

- cohort interpolation

- age-group interpolation.

- Determine weighting for final age/sex estimates.

- Determine race share estimates by interpolation.
STEP 3: Develop Estimates for County Census Divisions

- Standardize CCDs for HEDR use

- Calculate intercensus estimates for each CCD 1940 to 1970:

- Interpolate population shares between the two census counts bracketing a particular year.

- Multiply each CCD's county population share by the appropirate ratio-correlation intercensus estimate generated in Step 1.

- Use age/sex/race distributions for the county as a whole to determine CCD distributions.

FIGURE 2. Overview of Methodology Used to Develop HEDR Phase I Population Estimates 


$$
\begin{aligned}
& D=\text { deaths } \\
& I=\text { in-migration } \\
& 0=\text { out-migration. }
\end{aligned}
$$

The chalienge for demographers is to gather information or develop estimates for each of the components of population change. By convention, most U.S. demographic databases are maintained on the county ievel. For instance, reliable annual birth and deaths records are maintained for all U.S. counties but are not generally available for subcounty areas such as cities and towns. Unlike birth and death data, no county-level migration database is maintained and updated yearly.

As a result, the real challenge for estimating population in the U.S. is to develop models that accurately estimate county in- and out-migration for a particular county. Migration is a particularly important component of population change for HEDR demographic work because the study area has historically been characterized by rapid in- and out-migration due to the effects of war mobilization. For instance, over 34,000 construction workers migrated into Benton County in 1944 alone.

To develop the needed estimates for the HEDR Project, many different information sources were researched. The most important information sources are briefly summarized below.

\section{Information Sources for HEDR Population Estimation}

HEDR staff collected a wide variety of demographic data to help prepare population estimates, including U.S. census data; public-use microdata; vital statistics (i.e., birth and death data); special censuses conducted by state or local agencies; independent estimates provided by state and federal agencies; and symptomatic indicator data (e.g., schooi enroliment and voter registration data). Each data source is described in the following sections.

\section{U.S. Census Data}

A vast amount of demographic information is available from the censuses conducted by the U.S. Census Bureau every 10 years. To date, we have collected the 1940, 1950, 1960, 1970, and 1980 census publications for the 
states of Washington, Idaho, and Oregon. These documents provide totai population counts for al1 U.S. counties broken down by age, sex, and race for census years. Thus, they provide the benchmark data needed for the various estimation techniques discussed later in this paper. Census statistics are also provided in these publications for census subdivisions and towns and cities with populations larger than 1000 .

In addition to decennial information, we have obtained U.S. Census special reports on substate migration and subcounty estimation techniques. These U.S. Census data constitute the most comprehensive source of demographic information for the HEDR study area.

\section{Public-Use Microdata Sample}

These data collections were created through a joint effort of the U.S. Census Bureau and the Center for Demography and Ecology at the University of wisconsin. The collections contain information from a stratified sample of $1 \%$ of the households represented in the census. These files contain information on location and composition of the households, including such variables as nativity, marital status, number of children, occupation, education, employment status, and income. Data from these files are available back to the 1940 census.

\section{Vital Statistics Information}

We have collected annual birth and death staistics for all counties in the Phase I HEDR study area. Birth and death data were taken from the annua? vital rates summaries published by the U.S. government.

\section{Federal and State Population Estimates}

The states of Washington, Oregon, and Idaho have periodically published county and city population estimates. We have received documented estimates for Oregon counties dating back to 1930 and are currently collecting estimates for counties in Washington and Idaho. In addition to state estimates, the U.S. Census Bureau has routinely produced estimates of county population (using a variety of methods) since 1960. However, few county intercensus estimates for the time period 1940 through 1960 were located. 


\section{Symptomatic Information Sources}

We collected symptomatic information sources required for the component method II and ratio-correlation methods discussed later in this document. More specifically, we gathered school enrollment, voter registration, auto registration, employment, and vital statistics data for every county in Washington State. The sources for these data varied by state, but generaily they came from annual reports published by state agencies.

\section{Evaluation of Existing Data}

After reviewing all of the demographic information available, we determined that no reliable standardized county estimates were available for the HEDR Phase I study area for the years 1940 to 1960. Prior to the 1960s, demographic estimation efforts focussed on developing sound state estimates; county population estimates were routinely linearly interpolated from census year to census year. The intercensus estimates that did exist tended to be projections into the future from a particular time in history, and as a result they were unable to take into account pre-and post census information. Furthermore the estimates that were developed contained none of the age-sex-race detail needed for the HEDR Project. For this reason we deemed it necessary to produce our own county and sub-county estimates. Fortunately, as pointed out earlier, several techniques that have been refined over the last several decades produce accurate county-level population estimates by accurately modelling in- and out-migration. The sections below describe the three most commonly used methods applied to develop county population estimates (and the ones we adopted for the HEDR Project): ratio-correlation, component II, and vital rates.

\section{Ratio-Correlation Population Estimation Method}

This method involves the construction of a regression equation that models the relationship between symptomatic indicators (e.g., number of births, auto registrations, voter registration, etc.) and total population. For example, to estimate a county population, the regression equation expresses the relationship between 1) change over the previous intercensal period in the county's share of the state total for several symptomatic indicators and 
2) the change in the county's share of the state population (Shryock and Siegel 1976). The ratio correlation method uses observed temporal changes in the county proportions of symptomatic indicators to estimate county population totals (Swanson 1980). These steps may be represented symbolically as follows:

$$
\frac{\text { Proportion of total state population in county } i, 1960}{\text { Proportion of total state population in county } i, 1950}=\frac{\left(\frac{P_{\mathrm{c}}}{P_{\mathrm{s}}}\right)^{1960}}{\left(\frac{P_{\mathrm{c}}}{P_{\mathrm{s}}}\right)^{1950}}
$$

The independent variables, such as births, are expressed in the same manner:

$$
\frac{\text { Proportion of total state population in ccunty } i, 1960}{\text { Proportion of total state population in county } i, 1950}=\frac{\left(\frac{B_{\mathrm{c}}}{B_{\mathrm{s}}}\right)^{1960}}{\left(\frac{B_{\mathrm{c}}}{B_{\mathrm{s}}}\right)^{1559}}
$$

The validity of this method is based on the assumption that the observed statistical relationship between the independent and dependent variables in the past intercensal period will persist in the postcensal period (Shryock and Siegel 1976). Once the ratio-correlation mode? is constructed, the known ratio of proportions for each of the symptomatic indicator variables is multiplied by the appropriate regression coefficient. This step results in unadjusted county population estimates for each county. Finally, these unadjusted estimates are controlled to an independent state population estimate resulting in adjusted county estimates [for further explanation, see Namboodiri (1972); Shryock and Siegel (1976, pp. 428-429); and Crosetti and Schmitt (1956)]. These steps are discussed in more detail in Appendix $C$.

The ratio-correlation method is routinely used by the U.S. Census Bureau and has been used by the state of Washington since the 1970s. Because this method makes use of such a wide variety of symptomatic indicators, we believe it produces the most accurate county population estimates for the HEDR study 
area. In addition, because the estimates we produce are anchored by the postand pre-census numbers, we can be confident the estimates will be fairiy accurate. Estimates were prepared only for the years 1940-1970 because the Washington State Office of Financial Management began producing ratio correlation estimates for Washington counties in 1970.

In developing the ratio-correlation model, we decided to try to keep the model parsimonious and to use the same variables for all three decades to ensure model consistency. The predictor variables included county births, deaths, school enroliment, and registered voters. These variables were chosen because they were the only symptomatic indicators available for each of the three decades for which estimates were prepared. The ratio-correlation model used in preparing county estimates is reproduced in Table 1 . Each of the models can be conceptualized as either estimating the parameters of a sample of a larger universe or as calculating actual population measures (since we have complete data for every county in Washington). For the purpose of this report we have chosen the former interpretation. The fact that the $R^{2}$ (the proportion of the original equation that is accounted for by the regression equation) for all three models is larger than 0.90 indicates that the models do an excellent job of explaining variance. Births, school enrollment, and registered voters proved to be significant predictors of population in all three models. Deaths were not a significant predictor, although the negative relationship reported for all three decades was expected (i.e., as the number of deaths increased, total population decreased). The standard errors are recorded in parenthesis and are interpreted to be low.

In summary, the statistics reported in Table I suggest that our models do an excellent job of predicting population. In reviewing the above results it is important to point out that, unlike most regression analyses, the purpose of constructing these models was to predict population, not to estimate parameters. Therefore, a comparison of the population estimates developed with the ratio-correlation models and actual census counts is the soundest indicator of how "good" the previously described models are. This type of comparison is provided in the "Results" section of this report. 


\section{TABLE 1. Ratio-Correlation Model (OLS) for Washington
Counties by Decade $(a)(b)$}

\begin{tabular}{|c|c|c|c|}
\hline & $1940-50$ & $1950-60$ & $1960-70$ \\
\hline Intercept & $0.015(.049)$ & $0.08 \mathrm{~J}(.040)$ & $0.037(.062)$ \\
\hline Births & $0.153(.048)^{(c)}$ & $0.139(.040)^{(c)}$ & $0.259(.052)^{(c)}$ \\
\hline Deaths & $-0.035(.063)$ & $-0.005(.048)$ & $-0.033(.043)$ \\
\hline School Enrollment & $0.366(.060)^{(c)}$ & $0.254(.061)^{(\mathrm{c})}$ & $0.197(.079)^{(d)}$ \\
\hline Registered Voters & $0.500(.065)^{(c)}$ & $0.543(.054)^{(\mathrm{c})}$ & $0.532(.092)^{(c)}$ \\
\hline $\mathrm{R}^{2}$ & 0.989 & 0.969 & 0.907 \\
\hline $\operatorname{Adj} R^{2}$ & 0.988 & 0.965 & 0.896 \\
\hline SEE ${ }^{\{e\}}$ & 0.045 & 0.040 & 0.042 \\
\hline
\end{tabular}

(a) The issue of multicollinearity is not addressed because the purpose of these models is prediction (i.e., population estimation), not explanation (see Berry and Feldman 1985:41).

(b) Technically, these independent variables are ratios of proportions of the various symptomatic indicators (See Equation 2).

(c) Significant to the 0.01 level.

(d) Significant to the 0.05 leve?.

(e) Standard error of estimate.

\section{Component Method II Population Estimation Method}

The component method II produces annual population estimates by adding an estimate for the general non-group quarters population under 65 , the group quarters population, the military population, and the over-65 population. For the HEDR estimates, the methodology outlined below is used to calculate the general non-group quarters (i.e., dormitories, prisons, etc.) population. This methodology is primarily based on two references: "Component Method II Computational Procedure for Estimating the Population of Counties" (New York State Department of Commerce 1979) and the "United States Department of Commerce Bureau of the Census Memorandum" (U.S. Bureau of the Census 1978). A third reference, The Methods and Materials of Demography (Shyrock and Siegel 1976), is used to make adjustments to the component method II results.

The over-65 population is normally estimated using Medicare records. For the HEDR estimates, no separate estimate is made of the over-65 population because Medicare records are not available for the 1940 s and 1950s. Estimates 
of the military and group quarter populations for Benton County are obtained from Hanford archive records. The military and group quarters population for the other counties in the study area are obtained from the decennial censuses.

To estimate the general non-group quarters population, the natural increase and net migrants from a census date to the estimate date are added to the total population at the census date. The net migrants are calculated with a procedure that uses annual school enrollment data (Grades 1 through 8; Ages 6.5 through 14.49) to estimate a population migration rate. The natura 1 increase for a population is the number of births minus the number of deaths. A detailed example of how this method was used to produce results for the HEDR Project is reproduced in Appendix D.

\section{Vital Rates Population Estimation Method}

The premise of the vital rates method is that the observed change (from census year to estimation year) in birth or death rates for a county will be roughly equivalent to the change experienced by the state as a whole, i.e., demographic change for a geographic subset mirrors change for the entire set (Rives and Serow 1984; p. 56). For the HEDR estimations, the birth and death rates for the state of Washington are used to estimate the State's county populations.

This procedure requires the following data:

- the number of births and deaths for the study area for the estimation year

- the number of births and deaths for the study area for the most recent census year

- the population of the study area in the most recent census year

- the number of births and deaths for the reference area for the most recent census year and the estimation year

- the population of the reference area for the most recent census year and the estimation year.

The general formula for these estimations is

$$
X /[(Y) *(Z)]=P O P
$$


where $x$ = number of births (deaths) in the study area for the estimation year

$Y=$ ratio of census year birth (death) rate in study area to census year birth (death) rate in the reference area

$Z$ = birth (death) rate of the reference area for the estimation year. Six estimates are calculated for each year. The estimates are based on birth rates, death rates, average of birth and death rate estimates, centered births, centered deaths, and the average of the centered estimates. Centered births and centered deaths are used to stabilize the randomness that occurs with vital statistics. For centered data in any given year, the number of births or deaths used is an average of three annual numbers: the year preceding the estimation year, the estimation year, and the year following the estimation year. The "Results" section of this report contains all the vital rates estimates developed by this method.

\section{Selection of Estimates for Use in Subsequent Steps}

The results from all three of these methods are contained in the "Results" section. Plots of population estimated by each of the three methods (see "Results" section) clearly demonstrate the relative stability of ratiocorrelation estimates compared with the vital rates and component method II estimates. This is partially explained by the fact that, unlike the other two methods, the ratio-correlation methods uses multipie indicators to model population change. The use of multiple indicators moderates the influence of a spurious fluctuation in any one indicator for any particular year.

Estimating Benton County population in the mid 1940 s was a particularly difficult challenge because of the rapid in and out migration of large numbers of construction workers. Estimates for 1944 for all of the models include the addition of 34,315 construction workers to the numbers generated by the different methods. Without this addition, all three of the methods significantly underestimated the number of people residing in Benton County (70,987 based on a special county census conducted that year by the census bureau). The underestimates are to be expected since all of the estimation techniques were developed to estimate permanent residents and not transient construction workers. Discussions with local residents and representatives of Jaycor Incorporated (a military research organization) suggest that the Hanford 
construction workforce was dominated by single males. None of the estimation methods discussed in this paper could have sensed the influx of this population because construction workers in the 1940s were likely childless (few births and school age children per capita), young and healthy (few deaths per capita), an'd mobile (unlikely to register to vote in Benton County). Therefore, in consultation with the demographics sub-committee of the HEDR Technical Steering Panel (TSP), the average number of construction workers for $1944(34,315)$ was added to the 1944 population estimates. Adding these figures to the estimates results in numbers much closer to the Benton County population recorded in the 1944 special census.

It was initially suggested that estimates developed by the three methods be averaged to arrive at a best estimate. However, because several TSP members expressed concern over this procedure, we decided to choose a best estimate from among the three estimates developed. We believe the ratiocorrelation estimates are the most accurate, based on theoretical and empirical evidence. Theoretically, the ratio-correlation method should produce better estimates than the other two methods because it uses the most information. Birth, death, voter registration, and school enrollment data are all used in the ratio-correlation models, while only school enrollment data are used in the component method $I 1$ model, and only birth and death data are used in the vital rates estimates.

Furthermore, the superiority of the ratio-correlation estimates over the other two methods can be demonstrated empirically. The 1944 special census of Benton County allows us to compare estimates generated by the three different methods with census information for an intercensus year (1944). Table 2 summarizes the percent difference between the estimates and the number generated by the special census. As mentioned earlier the estimates include the addition of 34,315 construction workers recorded in monthly Hanford work reports. The ratio-correlation estimate deviated only 3.33 percent from the census figure; the component method II and vital rates estimate deviated 12.61 and 18.49 percent, respectively. Estimates using the three methods were also prepared for the decennial census years 1950, 1960, and 1970. Table 3 contains the results of this comparison. Once again, the ratio-correlation 
TABLE 2. Comparison of 1944 Benton County Population Estimates and 1944 Benton County Special Census Population Total $(70,987)$

\begin{tabular}{lccc} 
& $\begin{array}{c}1944 \\
\text { Estimate }\end{array}$ & $\begin{array}{c}\text { Absolute Difference Between } \\
\text { Estimate and Special Census }\end{array}$ & $\begin{array}{c}\text { Percent } \\
\text { Difference }\end{array}$ \\
\cline { 2 - 4 } Ratio Correlation & 68,615 & 2,367 & 3.33 \\
Component Method II & 79,939 & 8,952 & 12.61 \\
Vital Rates & 57,815 & 13,123 & 18.49
\end{tabular}

TABLE 3. Deviation of Census Year Estimates from Actual Census Counts

\begin{tabular}{|c|c|c|c|c|c|c|c|c|c|}
\hline \multirow[b]{2}{*}{ County } & \multicolumn{3}{|c|}{1950} & \multicolumn{3}{|c|}{1960} & \multicolumn{3}{|c|}{1970} \\
\hline & $\begin{array}{c}\text { Ratio } \\
\text { Correlation }\end{array}$ & $\begin{array}{c}\text { Component } \\
\text { II } \\
\end{array}$ & $\begin{array}{l}\text { Vital } \\
\text { Rațes }\end{array}$ & $\begin{array}{c}\text { Ratio } \\
\text { Correlation }\end{array}$ & $\begin{array}{l}\text { Component } \\
\text { II } \\
\end{array}$ & $\begin{array}{l}\text { Vital } \\
\text { Rates }\end{array}$ & $\begin{array}{c}\text { Ratio } \\
\text { Correlation }\end{array}$ & $\begin{array}{c}\text { Component } \\
\text { 11 } \\
\end{array}$ & $\begin{array}{l}\text { Vital } \\
\text { Rates }\end{array}$ \\
\hline Adems & 9.72 & $3 \uparrow .57$ & 5.16 & 0.36 & 6.05 & 12.32 & 2.84 & 12.70 & 14.10 \\
\hline Benton & 0.05 & 34.41 & 15.19 & 2.96 & 5.39 & 17.83 & 0.05 & 12.80 & 17.50 \\
\hline Frankt in & 0.25 & 29.37 & 4.36 & $1 . \pi 5$ & 2.36 & 14.41 & 1.60 & 12.66 & 7.08 \\
\hline Grant & 3.17 & 14.23 & 2.56 & 1.50 & 6.11 & 3.66 & 3.12 & 11.27 & 7.04 \\
\hline Kittitas & 1.73 & 24.62 & 0.97 & 3.69 & 2.03 & 7.54 & 8.06 & 11.84 & 13.31 \\
\hline K! ickitat & 2.72 & 28.92 & 10.20 & 3.97 & 5.81 & .80 & 0.04 & 10.26 & 1.60 \\
\hline Wall a Walla & 1.18 & 28.93 & 4.88 & 0.71 & 2.78 & 6.07 & 2.73 & 5.26 & 4.67 \\
\hline Yakina & 6.24 & 25.99 & 1.18 & $\underline{3.25}$ & $\underline{2.96}$ & 2.33 & 6.00 & 10.72 & $11.6 ?$ \\
\hline Average & 3.13 & 27.26 & 5.6 & 2.27 & 4.19 & 8.12 & 3.06 & 10.94 & 9.6 \\
\hline
\end{tabular}

estimates were more accurate than the estimates prepared by the other two methods. Ratio-correlation estimates deviated from the census numbers by 3.13 percent in 1950, 2.27 percent in 1960, and 3.06 percent in 1970 . (a) Component method II estimates deviated by large amounts: 27.26 percent, 4.19 percent, and 10.94 percent for the years 1950,1960 , and 1970 . The vital rates estimates deviated by 5.6 percent, 8.12 percent, and 9.6 percent for the same years. The above comparisons clearly indicate the superiority of the

(a) In the component II method, the difference between the census year estimate and the census count (referred to as the error of closure) is evenly spread out over the eight intercensus estimates. As a result, the intercensus year estimates are likely tc be much more accurate than estimate prepared for a census year. 
ratio-correlation estimates. Therefore, the ratio-correlation estimates are used as the control totals for the additional demographic work described below.

\section{STEP 2: DEVELOPING COUNTY-LEVEL AGE, SEX, AND RACE ESTIMATES}

Developing age, sex, and race estimates is a challenge because it requires that migration estimates be prepared by age group. At times, this requires judgement calls based on an intimate knowledge of local demographics. For this reason, we decided to sub-contract this piece of the work to Dr. Donald Pittenger.

\section{Age and Sex Estimation Methods}

Dr. Pittenger explored three models for estimating population by age-sex group: cohort-component estimation, cohort interpolation, and age-group interpolation. He used the ratio-correlation estimates developed in step 1 as his control totals. The following discussion is taken from his report, which is published separately (Pittenger 1991).

\section{Cohort-Component Estimation Model}

This model was explored because it could potentially provide estimates of the population remaining in the study area over various time spans. It can keep track of populations by arrival year, and shows how many in each cohort (a group that arrives in the same year by migration or birth) are still. in the area at each subsequent year. For instance, using this method it would be possible to estimate, by age and sex, the number of people who lived in Benton County throughout the period 1944-1947 as well as the number entering and leaving the county through birth, death, and migration over that span.

Unfortunately, after initial tests it was determined that it was not feasible to use this model for Phase I results even though it was developed, debugged, and tested. The reason it was not used was that initial test runs showed that 1) a retirement-age migration component was needed, and 2) calibrating the model to each county was prohibitively time consuming. 


\section{Cohort Interpolation}

Although this method is less-sophisticated than the cohort-component model, we believe it produced accurate estimates suitable for Phase I purposes. This method entails interpolating inter-decade rates of change for single-year age-sex groups (birth cohorts). For instance, the Grant County ratio of males age 46 in 1960 to males age 36 in 1950 was computed, the 10th root taken, and interpolation was performed by raising the root to the first through ninth powers and multiplying the result by the 1950 population.

Once interpolations were made, the trial populations were reduced to age-sex shares of the totals. These shares were then multiplied by the intercensal total population estimates (ratio-correlation estimates) to provide provisional age-sex estimates.

\section{Age-Group Interpolation}

This method simply i inearly interpolates between corresponding age-sex groups at the beginning and the end of each the decade. For instance, females who were age 27 in Benton County in 1960 would be subtracted from Benton County females who were age 27 in 1970, and the nterpolation involved taking tenths of the amount of change. As with the cohort interpolation method, the resulting populations were transformed into age-sex shares and multiplied by the control totals (ratio-correlation estimates)

\section{Final Age/Sex Estimates}

Final estimates were made by weighing the results of the two methods. In most cases, the cohort interpolation model was given a weight of 0.8 , and the age-group interpolation a weight of 0.2 . In Dr. Pittenger's opinion, this seemed to offer more realistic age detail for intercensal years by preserving the "lumpiness" of age groups for small areas as they passed through time. The final age and sex estimates developed by Dr. Pittenger are contained in his report (Pittenger 1991).

\section{Race Estimation Method}

Race share estimates, by age and sex groups, were produced by interpolating between census years. These shares were multiplied by intercensal 
population estimates. Race groups estimated were 1) whites, 2) non-whites, 3) Negroes/blacks, and 4) Indians. The age, sex, and race results developed by Dr. Pittenger are contained in his report (Pittenger 1991). It is important to note that due to serious census undercounting of Indians, Indian population estimates independent of census information are being prepared.

\section{STEP 3: DEVELOPING POPULATION ESTIMATES FOR COUNTY CENSUS DIVISIONS}

To provide finer resolution, HEDR estimates are reported by county subdivisions. The county sub-divisions used for the HEDR Project are based on the county census divisions (CCDS) developed by the U.S. Census Bureau. The census bureau developed CCDs based on population density and regional economic organization. The HEDR county subdivision boundaries are identical to those developed by the U.S. Census Bureau with the following three exceptions: 1) Each CCD was renamed with the first two letters of the respective county name in front of the division number (e.g., BEl is located in Benton County). 2) The Pasco North Division was divided into two sections (FR4 and FR5) to provide greater detail to an area that may have received relatively high levels of radioactive deposits due to its proximity to Hanford. 3) Several of the very small CCDs in Yakima County were aggregated into larger divisions. This was done to limit the number of CCDs in this county to a manageable number and keep it in rough equivalence to the number of CCDs in the other counties. For further discussion of the creation of HEDR CCDs, see Beck et al. (1990). Figure 3 illustrates the HEDR CCDs.

Intercensus population estimates were calculated for all CCDs within the HEDR study area for the years 1940 to 1970. Intercensus estimates were prepared by interpolating population shares between the two census counts bracketing a particular estimate year. Each CCD's county population share was then multiplied by the appropriate ratio-correlation intercensus estimate.

CCD boundaries changed several times during the 1940-1970 time span. To maintain consistency, the CCD boundaries reported in the 1970 census are used for all years. CCD boundaries for earlier decades were modified to make them consistent with the 1970 boundaries. Appendix E contains a description of how a1) the changes from census to census were addressed. The "Results" section 


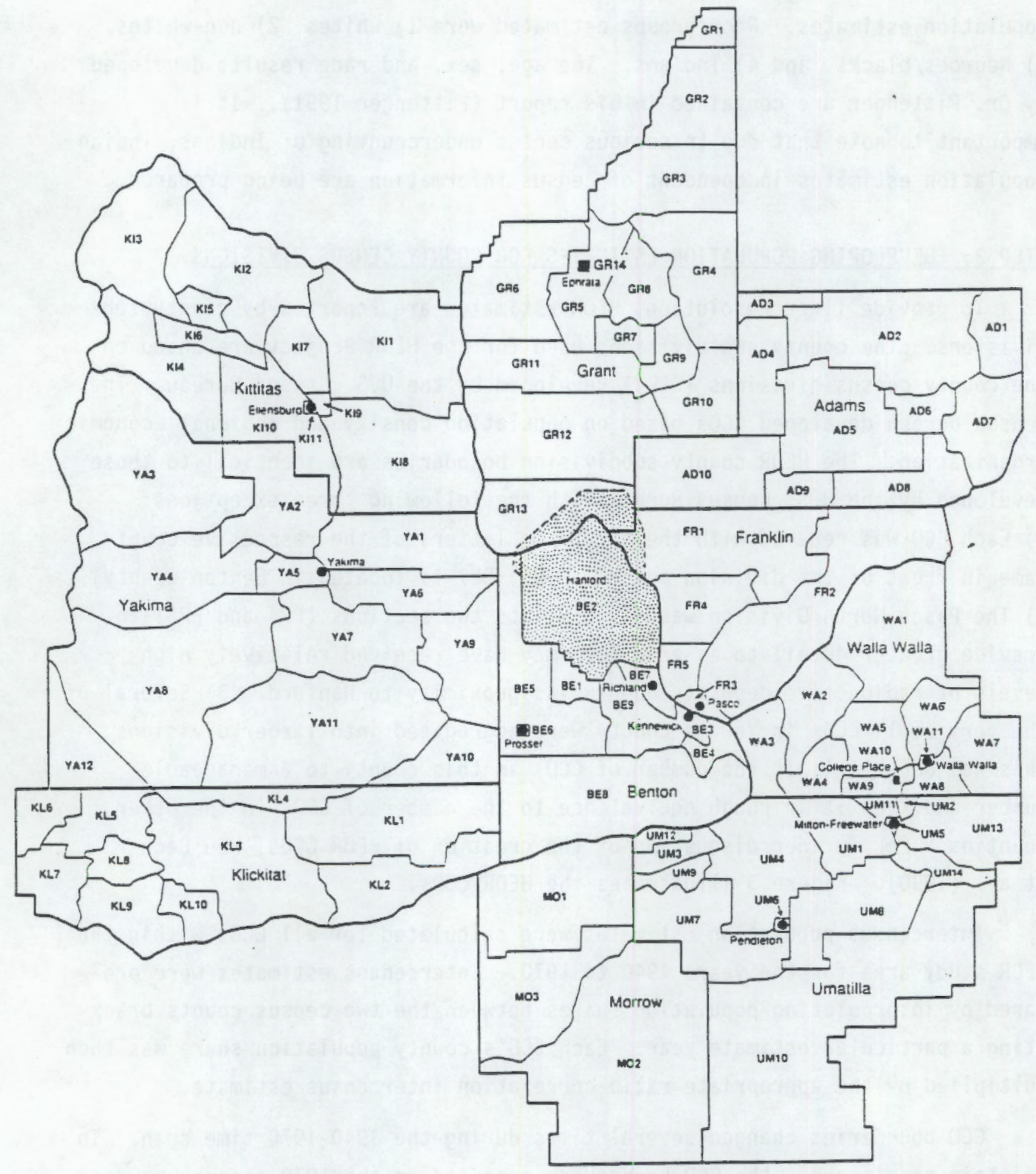

FIGURE 3. Hanford Environmental Dose Reconstruction County Census Divisions 
of this report contains the population estimates for each HEDR CCD. Age, sex, and race distributions for the CCD were assumed to be identical to the distributions developed for the county as a whole. 



\section{RESULTS}

Phase I HEDR population estimates consist of the following: 1) estimates from each of three methods for each county in Washington for each year from 1940 to 1970,2$)$ population estimates for each CCD in the HEDR Phase I study area for each year, and 3) age/sex/race population component estimates for each county in the HEDR Phase I study area for each year.

The Washington County estimates and the HEDR study area CCD estimates are reported here. The age/sex/race component estimates were prepared by Donald Pittenger and are given in a separate report (Pittenger 1991).

\section{COUNTY-LEVEL HEDR POPULATION ESTIMATES 1940 THOUGH 1970}

The results from a11 three of the methods used to estimate county populations are presented in this section. Estimates were prepared for all counties in Washington for the years 1940 through 1970. We did not prepare estimates for Dregon counties because official historical estimates were available from the Oregon State Center for Population Research and Census. Oregon estimates were most likely developed by some combination of straight-line interpolation and component method II procedures. (a)

Plots of the estimates from all three methods are given for each Washington county in the HEDR study area in Figures 4 through 11 . Figures 12 and 13 depict the Morrow and Umatilla estimates supplied by the Oregon State Center for Population Research and Census.

A complete listing of estimates for all Washington counties is given in Tables 4 a through $4 \mathrm{e}$. The listing is divided by year as follows:

$\begin{array}{ll}4 a & 1940-1946 \\ 4 b & 1947-1953 \\ 4 c & 1954-1960 \\ 4 d & 1961-1967 \\ 4 e & 1968-1970 .\end{array}$

(a) This is based on a phone conversation with the director of the Oregon State Center for Population Research and Census. 


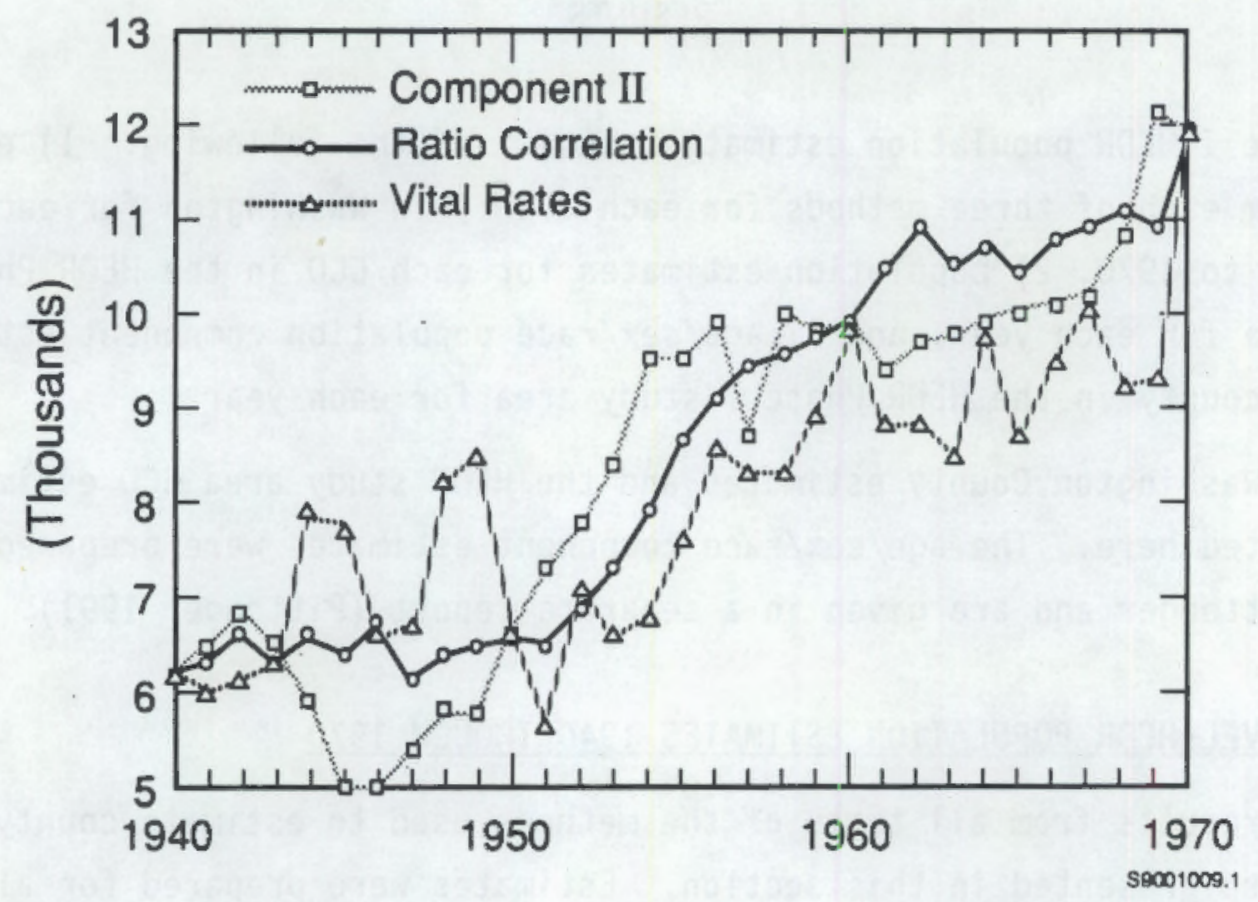

FIGURE 4. Adams County Population Estimates 1940 through 1970

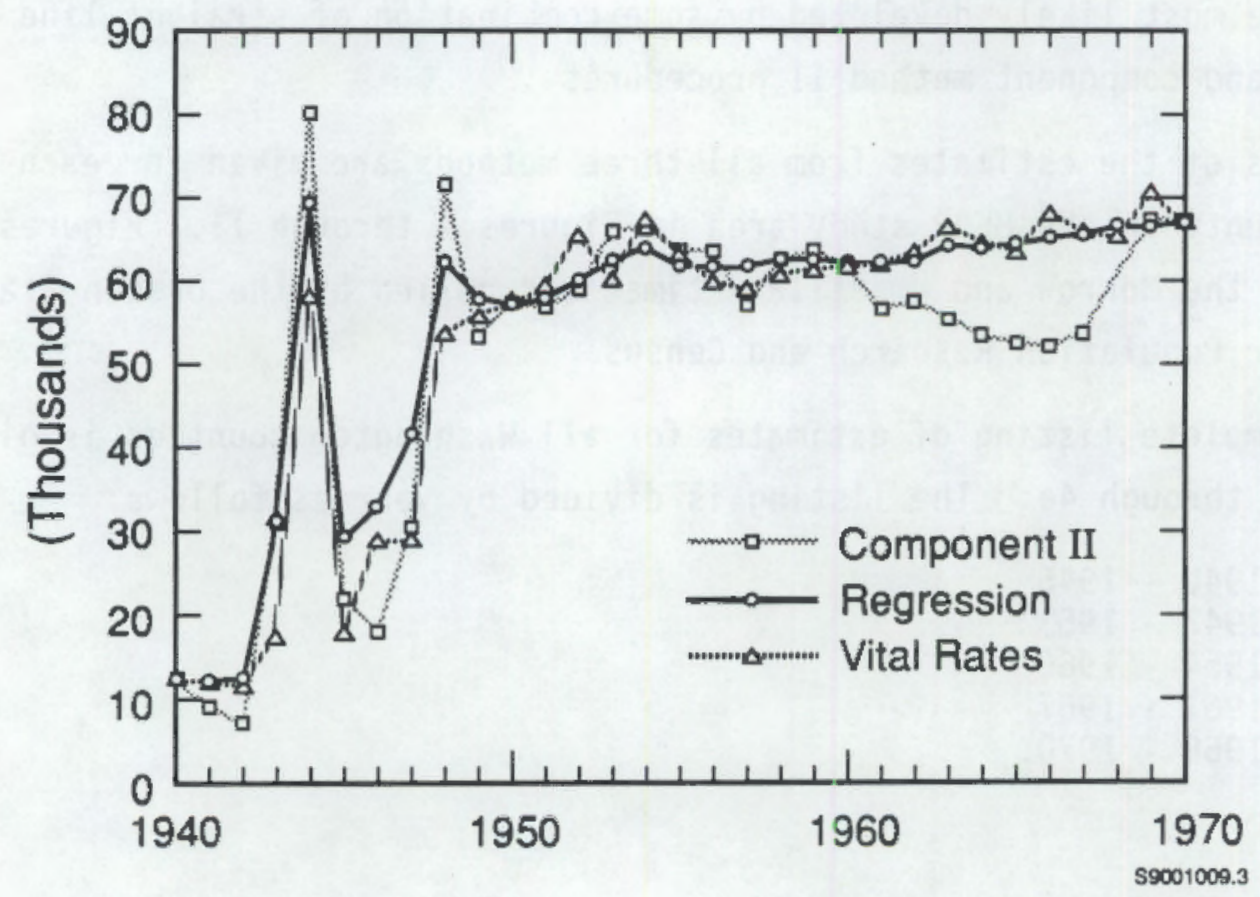

FIGURE 5. Benton County Population Estimates 1940 through 1970 


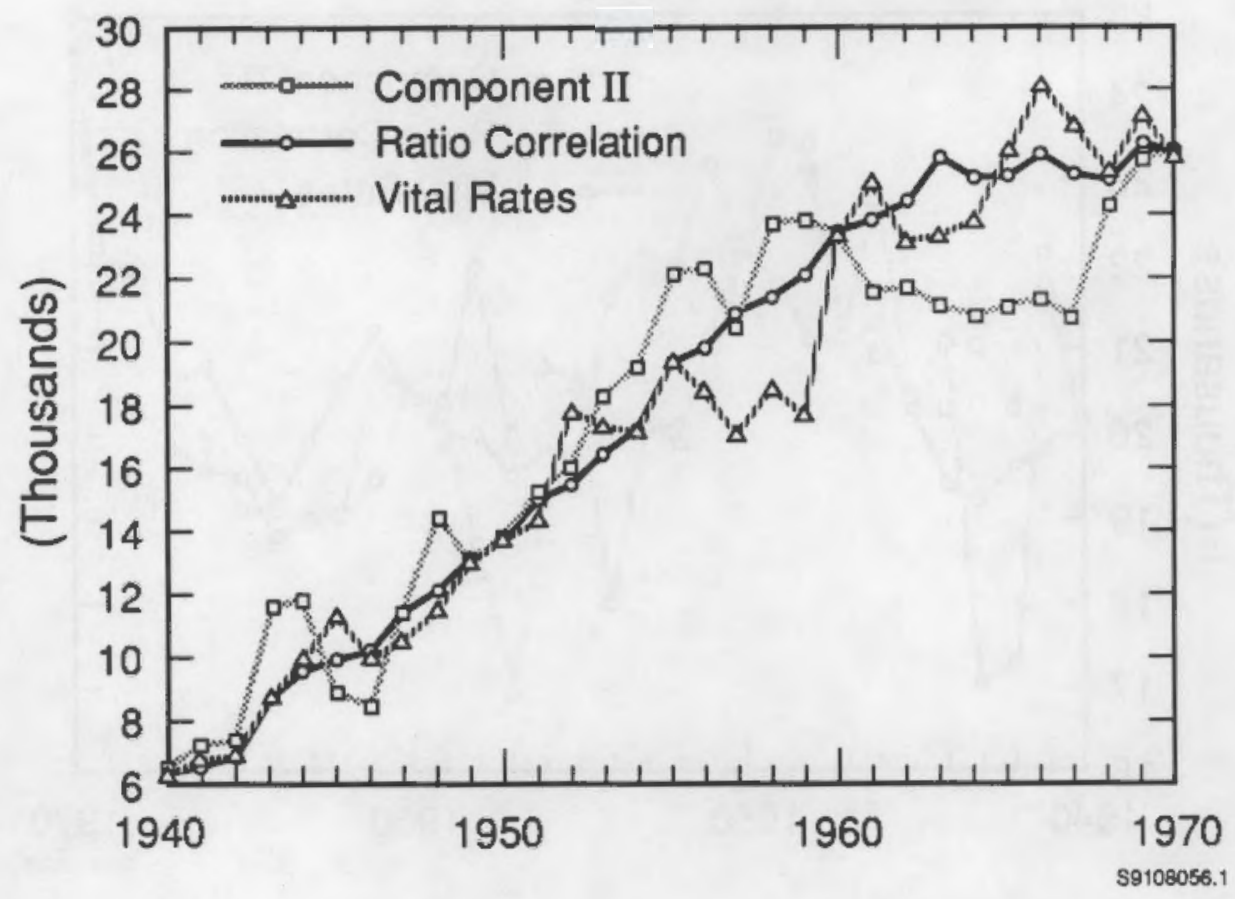

FIGURE 6. Frankl in County Population Estimates 1940 through 1970

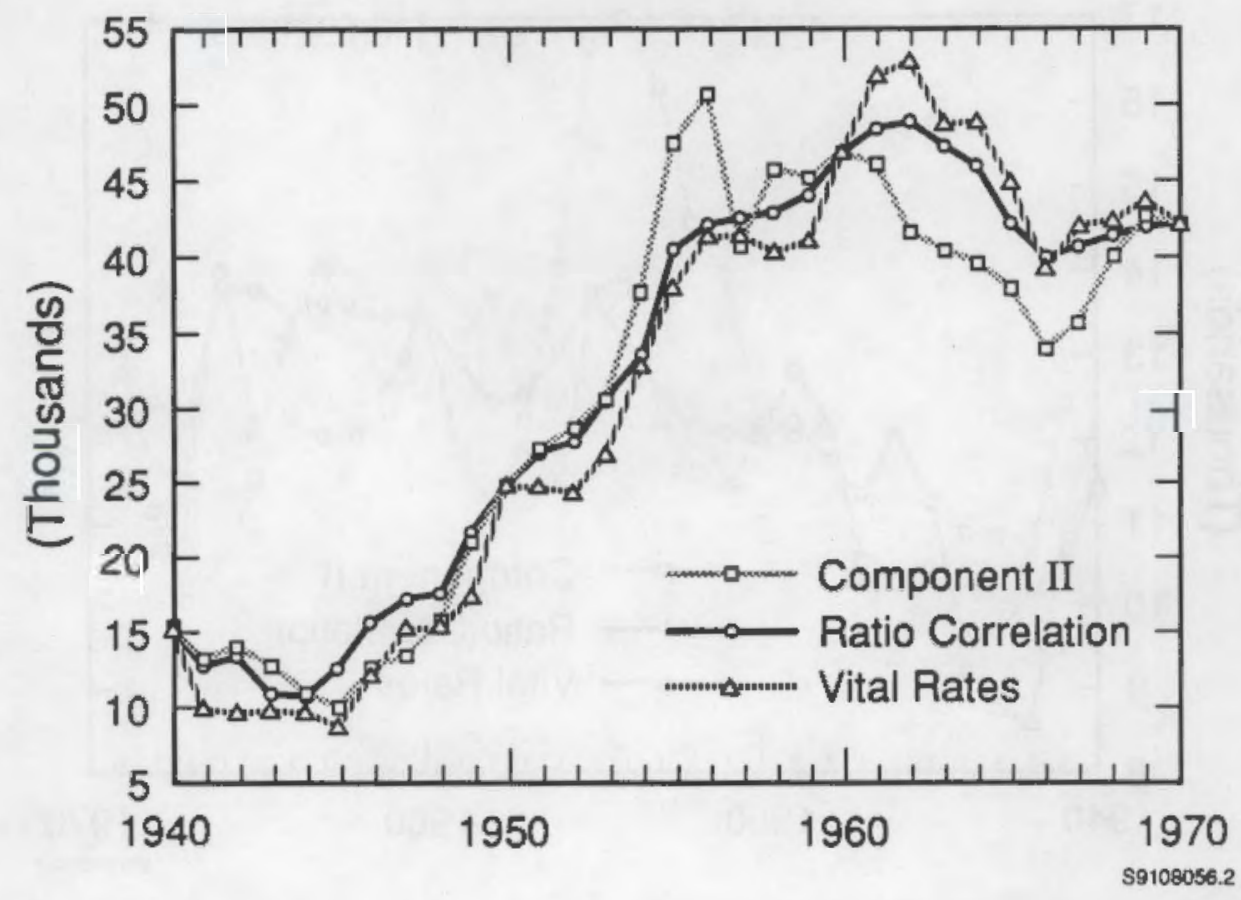

FIGURE 7. Grant County Population Estimates 1940 through 1970 


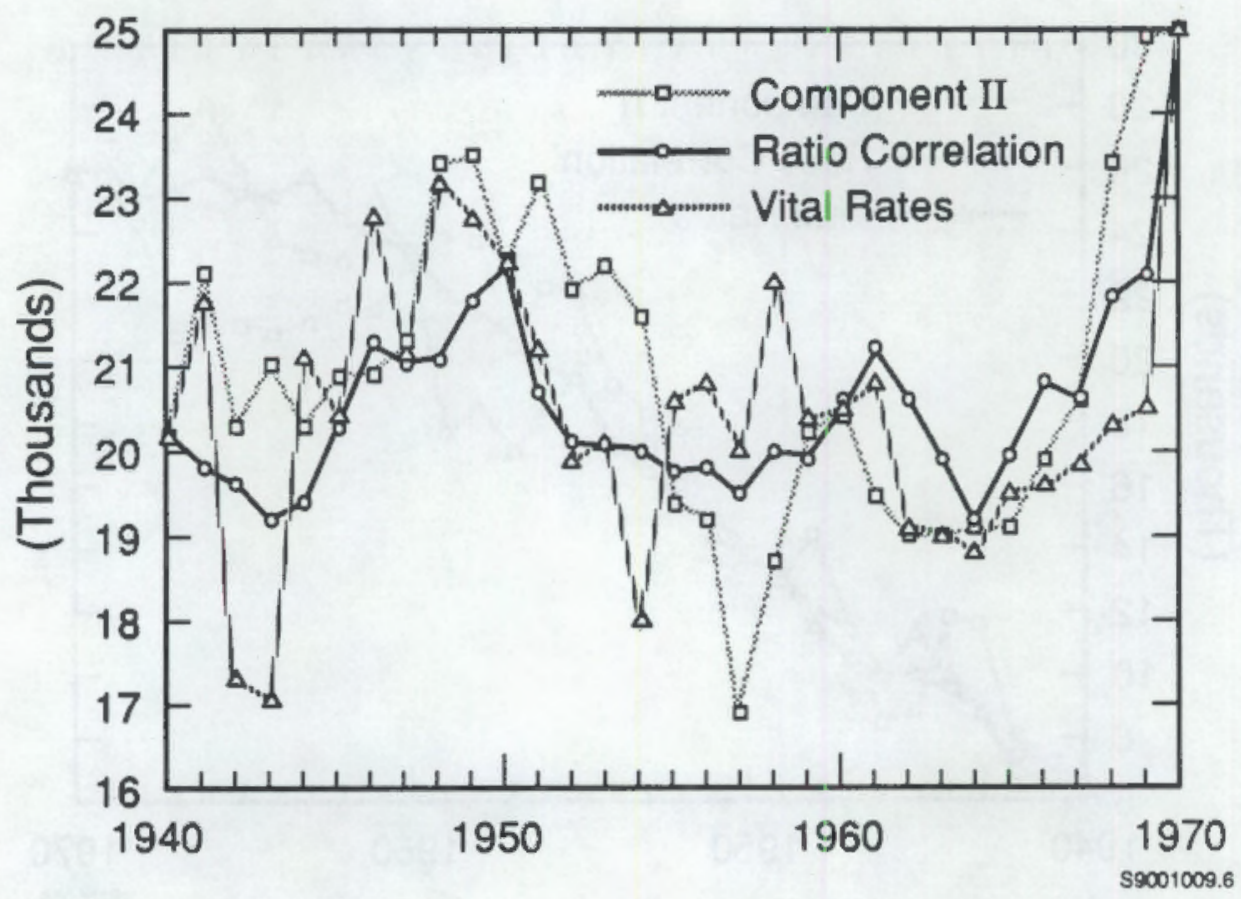

FIGURE 8. Kittitas County Population Estimates 1940 through 1970

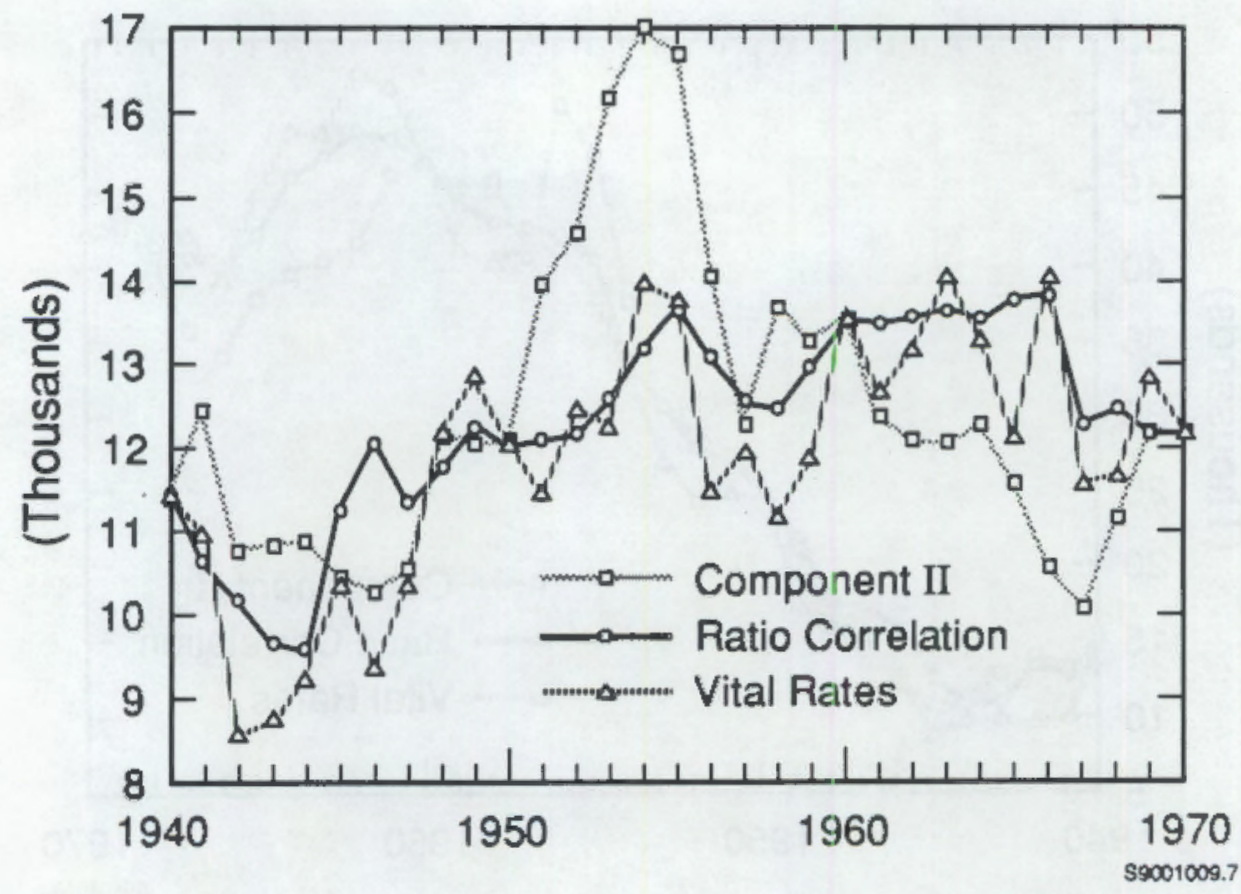

FIGURE 9. Klickitat County Population Estimates 1940 through 1970 


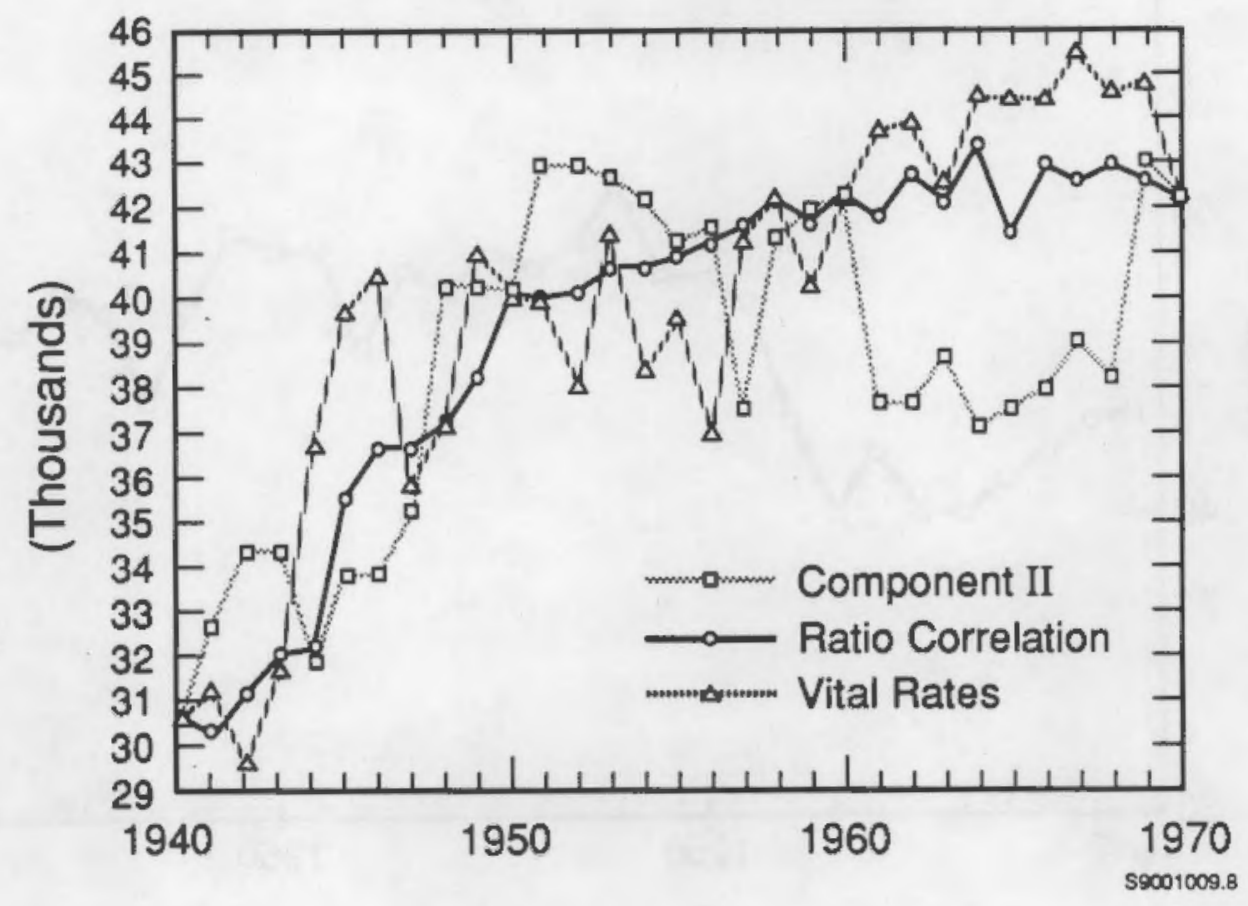

FIGURE 10. Walla Walla County Population Estimates 1940 through 1970

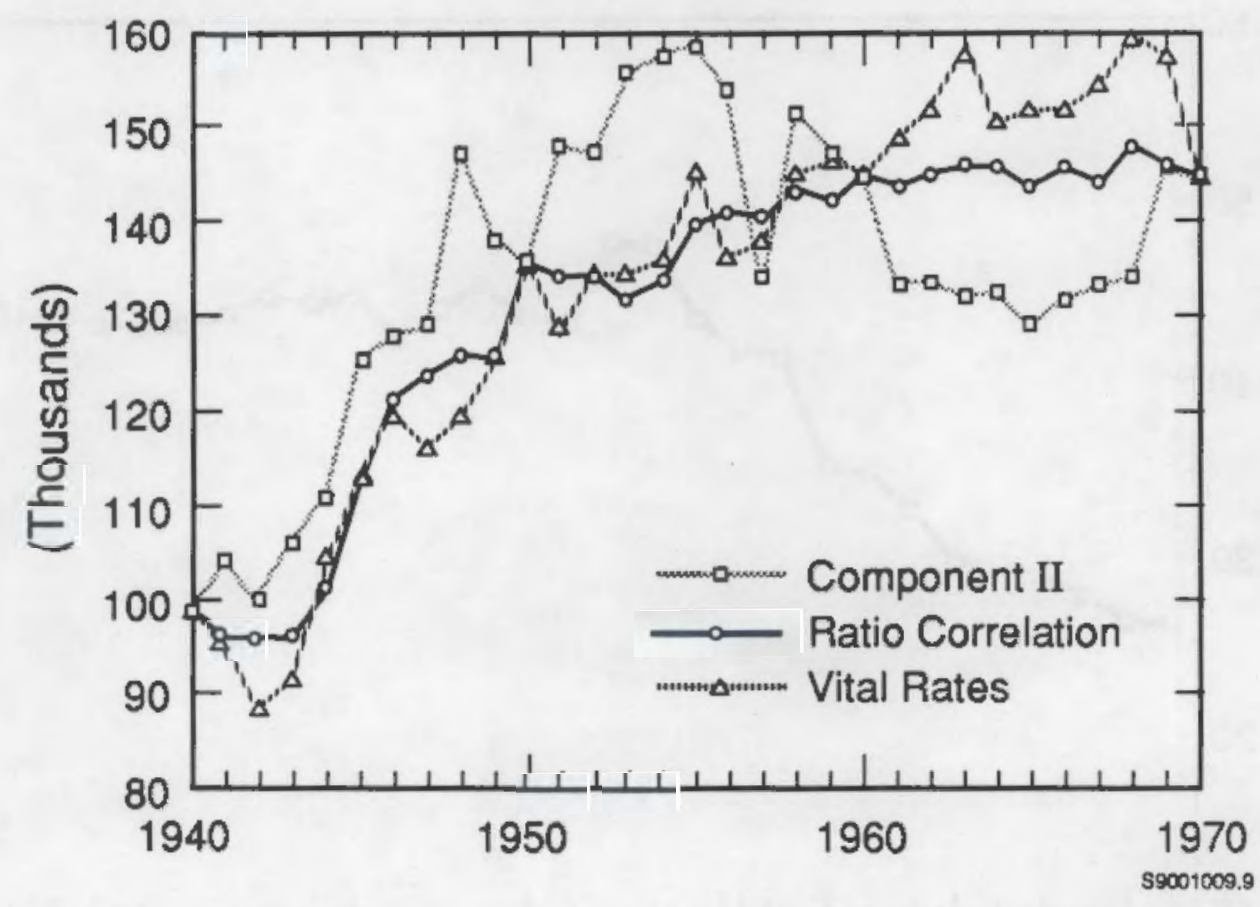

FIGURE 11. Yakima County Population Estimates 1940 through 1970 


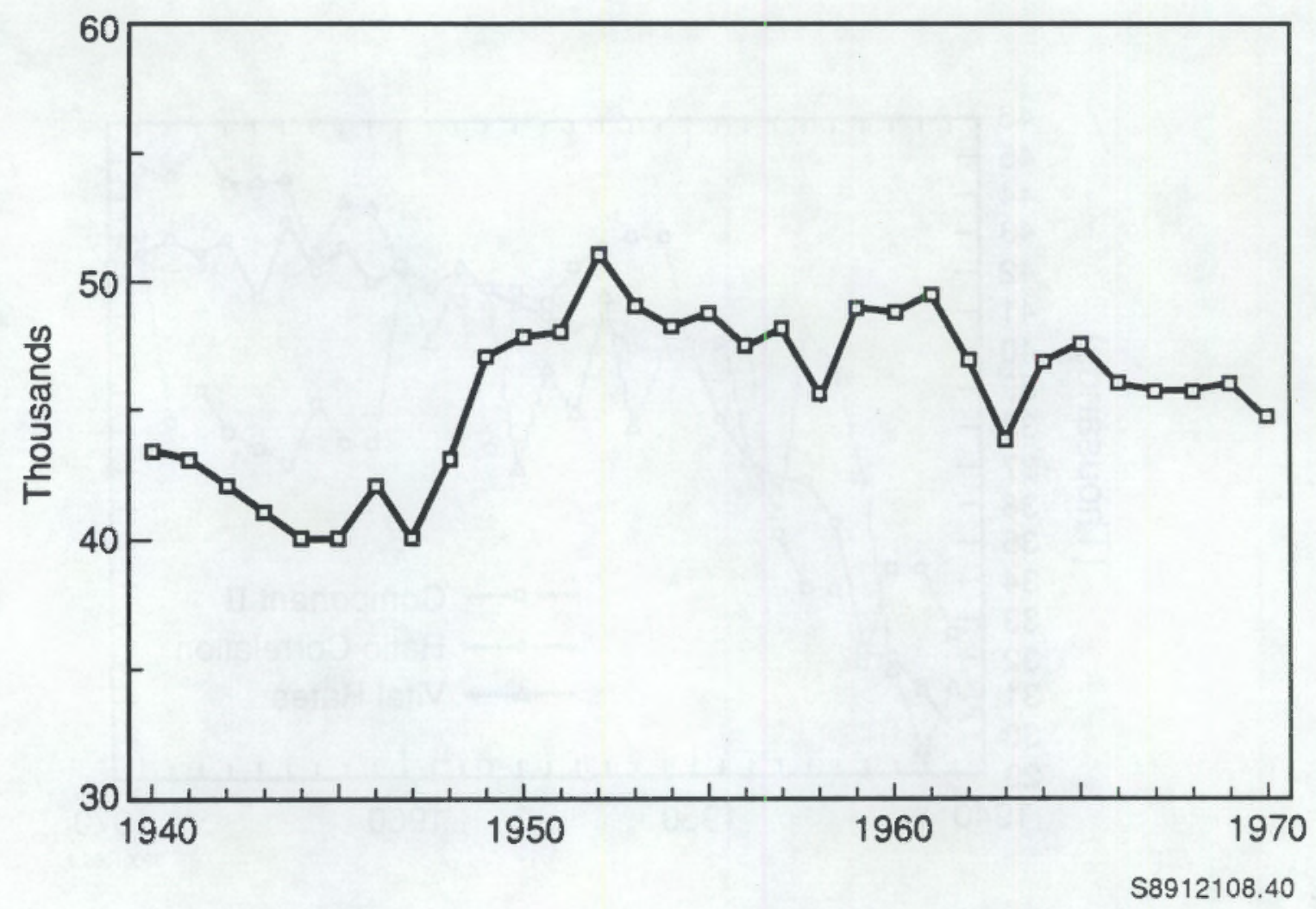

FIGURE 12. Morrow County Population Estimates 1940 through 1970

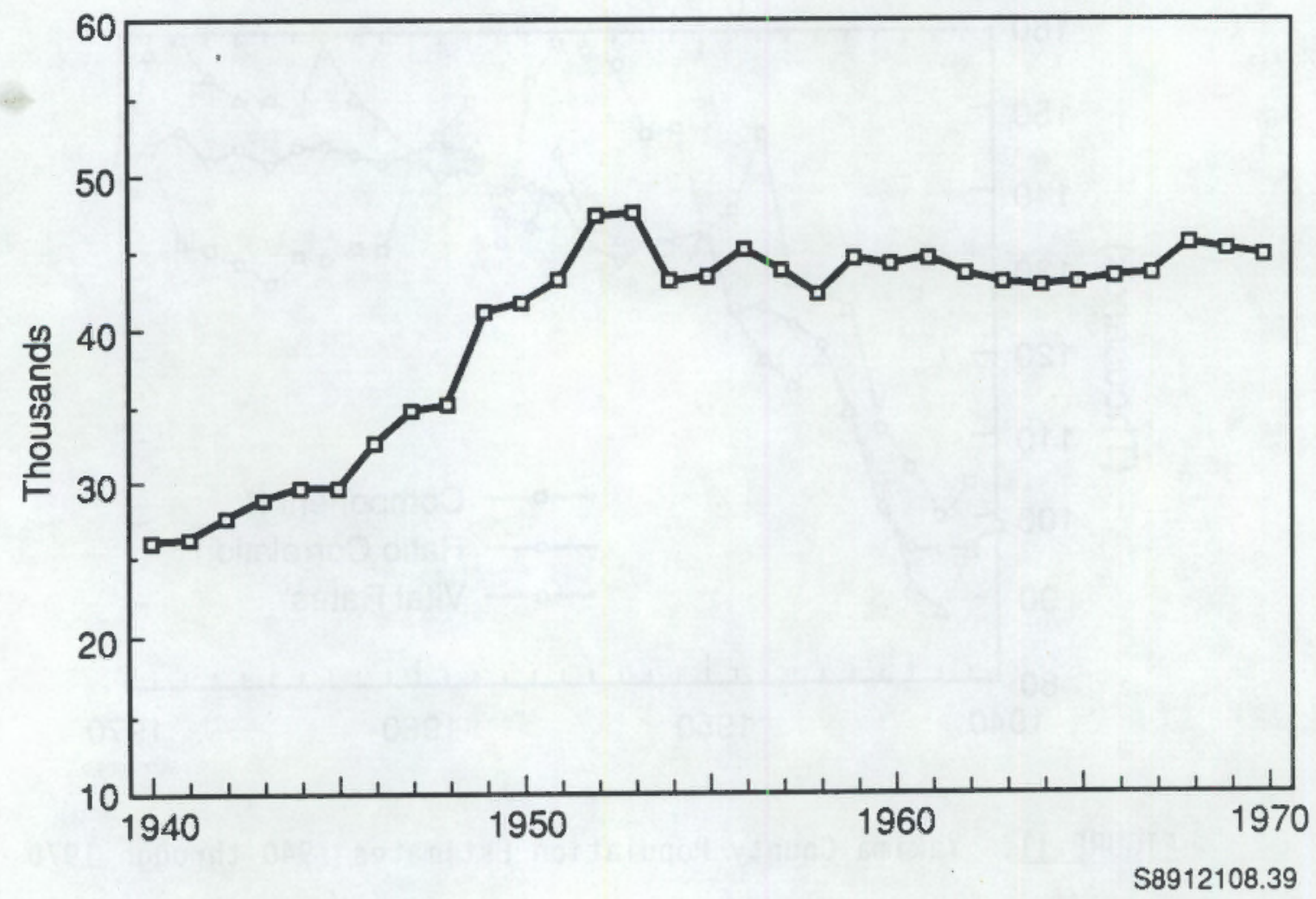

FIGURE 13. Umatilla County Population Estimates 1940 through 1970 
IABLE 4a. Population Estimates for Washington Counties $1941-1946^{(a)}$

\begin{tabular}{|c|c|c|c|c|c|c|c|c|}
\hline & & $1940^{(b)}$ & 1941 & 1942 & 1943 & 1944 & 1945 & 1946 \\
\hline ADAMS & $\begin{array}{l}\text { VITAL }{ }^{(c)} \\
\text { RATIO } \\
\text { COMPONENT II }\end{array}$ & $\begin{array}{l}6209 \\
6209 \\
6209\end{array}$ & $\begin{array}{l}6000 \\
6300 \\
6500\end{array}$ & $\begin{array}{l}6100 \\
6600 \\
6800\end{array}$ & $\begin{array}{l}6300 \\
6300 \\
6500\end{array}$ & $\begin{array}{l}7900 \\
6600 \\
5900\end{array}$ & $\begin{array}{l}7700 \\
6400 \\
5000\end{array}$ & $\begin{array}{l}6600 \\
6700 \\
5000\end{array}$ \\
\hline ASOTIN & $\begin{array}{l}\text { VITAL } \\
\text { RATIO } \\
\text { COMPONENT II }\end{array}$ & $\begin{array}{l}8365 \\
8365 \\
8365\end{array}$ & $\begin{array}{r}7300 \\
8400 \\
10100\end{array}$ & $\begin{array}{l}9300 \\
8500 \\
9700\end{array}$ & $\begin{array}{l}7200 \\
7800 \\
9500\end{array}$ & $\begin{array}{l}8700 \\
8300 \\
9300\end{array}$ & $\begin{array}{l}9100 \\
9100 \\
7600\end{array}$ & $\begin{array}{l}8100 \\
9400 \\
7700\end{array}$ \\
\hline BENTON & $\begin{array}{l}\text { VITAL } \\
\text { RATIO } \\
\text { COMPONENT II }\end{array}$ & $\begin{array}{l}12053 \\
12053 \\
12053\end{array}$ & $\begin{array}{r}12300 \\
12100 \\
8900\end{array}$ & $\begin{array}{r}11500 \\
12300 \\
6800\end{array}$ & $\begin{array}{l}12800 \\
24600 \\
26400\end{array}$ & $\begin{array}{l}70987 \\
70987 \\
70987\end{array}$ & $\begin{array}{l}18109 \\
30300 \\
21609\end{array}$ & $\begin{array}{l}28761 \\
32900 \\
17861\end{array}$ \\
\hline CHELAN & $\begin{array}{l}\text { VITAL } \\
\text { RATIO } \\
\text { COMPONENT II }\end{array}$ & $\begin{array}{l}34412 \\
34412 \\
34412\end{array}$ & $\begin{array}{l}34400 \\
34200 \\
37300\end{array}$ & $\begin{array}{l}34000 \\
34600 \\
37700\end{array}$ & $\begin{array}{l}31800 \\
33100 \\
37200\end{array}$ & $\begin{array}{l}34500 \\
34200 \\
37500\end{array}$ & $\begin{array}{l}38000 \\
35300 \\
38600\end{array}$ & $\begin{array}{l}41900 \\
38700 \\
39900\end{array}$ \\
\hline ALLAM & $\begin{array}{l}\text { VITAL } \\
\text { RATIO } \\
\text { COMPONENT II }\end{array}$ & $\begin{array}{l}21848 \\
21848 \\
21848\end{array}$ & $\begin{array}{l}21500 \\
21800 \\
22000\end{array}$ & $\begin{array}{l}20800 \\
22400 \\
22700\end{array}$ & $\begin{array}{l}20800 \\
22000 \\
23500\end{array}$ & $\begin{array}{l}22100 \\
21400 \\
20500\end{array}$ & $\begin{array}{l}22200 \\
23300 \\
22200\end{array}$ & $\begin{array}{l}23900 \\
24300 \\
22300\end{array}$ \\
\hline ARK & $\begin{array}{l}\text { VITAL } \\
\text { RATIO } \\
\text { COMPONENT II }\end{array}$ & $\begin{array}{l}49852 \\
49852 \\
49852\end{array}$ & $\begin{array}{l}55700 \\
52800 \\
58600\end{array}$ & $\begin{array}{l}66500 \\
67200 \\
95700\end{array}$ & $\begin{array}{r}92600 \\
91500 \\
127400\end{array}$ & $\begin{array}{r}99900 \\
96500 \\
135200\end{array}$ & $\begin{array}{r}97000 \\
96400 \\
112300\end{array}$ & $\begin{array}{l}90500 \\
92300 \\
93100\end{array}$ \\
\hline LUMBIA & $\begin{array}{l}\text { VITAL } \\
\text { RATIO } \\
\text { COMPONENT II }\end{array}$ & $\begin{array}{l}5549 \\
5549 \\
5549\end{array}$ & $\begin{array}{l}6300 \\
5500 \\
5900\end{array}$ & $\begin{array}{l}5600 \\
5300 \\
5500\end{array}$ & $\begin{array}{l}5000 \\
4600 \\
5000\end{array}$ & $\begin{array}{l}6700 \\
4600 \\
5000\end{array}$ & $\begin{array}{l}6500 \\
4900 \\
5000\end{array}$ & $\begin{array}{l}5700 \\
5200 \\
4700\end{array}$ \\
\hline WLITZ & $\begin{array}{l}\text { VITAL } \\
\text { RATIO } \\
\text { COMPONENT II }\end{array}$ & $\begin{array}{l}40155 \\
40155 \\
40155\end{array}$ & $\begin{array}{l}40600 \\
41500 \\
46100\end{array}$ & $\begin{array}{l}41100 \\
43500 \\
47500\end{array}$ & $\begin{array}{l}36300 \\
40200 \\
45000\end{array}$ & $\begin{array}{l}38100 \\
40400 \\
43500\end{array}$ & $\begin{array}{l}41600 \\
44600 \\
45800\end{array}$ & $\begin{array}{l}43700 \\
46600 \\
44500\end{array}$ \\
\hline UGLAS & $\begin{array}{l}\text { VITAL } \\
\text { RATIO } \\
\text { COMPONENT II }\end{array}$ & $\begin{array}{l}8651 \\
8651 \\
8651\end{array}$ & $\begin{array}{l}8800 \\
8400 \\
9200\end{array}$ & $\begin{array}{l}7000 \\
8400 \\
8500\end{array}$ & $\begin{array}{l}8600 \\
8000 \\
7800\end{array}$ & $\begin{array}{l}9100 \\
8400 \\
8000\end{array}$ & $\begin{array}{l}8100 \\
8400 \\
6200\end{array}$ & $\begin{array}{l}9300 \\
9500 \\
6800\end{array}$ \\
\hline RRY & $\begin{array}{l}\text { VITAL } \\
\text { RATIO } \\
\text { COMPONENT II }\end{array}$ & $\begin{array}{l}4701 \\
4701 \\
4701\end{array}$ & $\begin{array}{l}4300 \\
4100 \\
4800\end{array}$ & $\begin{array}{l}3700 \\
4000 \\
4200\end{array}$ & $\begin{array}{l}3700 \\
3300 \\
3500\end{array}$ & $\begin{array}{l}4000 \\
3100 \\
3100\end{array}$ & $\begin{array}{l}2500 \\
3600 \\
3200\end{array}$ & $\begin{array}{l}2900 \\
4100 \\
3700\end{array}$ \\
\hline ANKLIN & $\begin{array}{l}\text { VITAL } \\
\text { RATIO } \\
\text { COMPONENT II }\end{array}$ & $\begin{array}{l}6307 \\
6307 \\
6307\end{array}$ & $\begin{array}{l}6800 \\
6400 \\
7100\end{array}$ & $\begin{array}{l}6800 \\
6700 \\
7200\end{array}$ & $\begin{array}{r}8700 \\
8700 \\
11500\end{array}$ & $\begin{array}{r}9900 \\
9500 \\
11700\end{array}$ & $\begin{array}{r}11200 \\
9800 \\
8700\end{array}$ & $\begin{array}{r}9900 \\
10100 \\
8300\end{array}$ \\
\hline IF IELD & $\begin{array}{l}\text { VITAL } \\
\text { RATIO } \\
\text { COMPONENT I I }\end{array}$ & $\begin{array}{l}3383 \\
3383 \\
3383\end{array}$ & $\begin{array}{l}2600 \\
3300 \\
3700\end{array}$ & $\begin{array}{l}2500 \\
3100 \\
3000\end{array}$ & $\begin{array}{l}2600 \\
3100 \\
2900\end{array}$ & $\begin{array}{l}2600 \\
3200 \\
3000\end{array}$ & $\begin{array}{l}2600 \\
3200 \\
2800\end{array}$ & $\begin{array}{l}2600 \\
3400 \\
2900\end{array}$ \\
\hline
\end{tabular}


IABLE 4a. (contd)

\begin{tabular}{|c|c|c|c|c|c|c|c|c|}
\hline & & $1940^{(b)}$ & 1941 & 1942 & -1943 & 1944 & 1945 & 1946 \\
\hline GRANT & $\begin{array}{l}\text { VITAL } \\
\text { RATIO } \\
\text { COMPONENT II }\end{array}$ & $\begin{array}{l}14668 \\
14668 \\
14668\end{array}$ & $\begin{array}{r}9600 \\
12100 \\
12700\end{array}$ & $\begin{array}{r}9100 \\
12800 \\
13400\end{array}$ & $\begin{array}{r}9400 \\
10200 \\
12200\end{array}$ & $\begin{array}{r}9100 \\
9800 \\
10400\end{array}$ & $\begin{array}{r}8300 \\
12000 \\
9500\end{array}$ & $\begin{array}{l}11800 \\
15000 \\
12200\end{array}$ \\
\hline $\begin{array}{l}\text { GRAYS } \\
\text { HARBOR }\end{array}$ & $\begin{array}{l}\text { VITAL } \\
\text { RATIO } \\
\text { COMPONENT II }\end{array}$ & $\begin{array}{l}53166 \\
53166 \\
53166\end{array}$ & $\begin{array}{l}52300 \\
52300 \\
57600\end{array}$ & $\begin{array}{l}50400 \\
52100 \\
53300\end{array}$ & $\begin{array}{l}45600 \\
49400 \\
52600\end{array}$ & $\begin{array}{l}48800 \\
49900 \\
51300\end{array}$ & $\begin{array}{l}51600 \\
51700 \\
50400\end{array}$ & $\begin{array}{l}55800 \\
55000 \\
51100\end{array}$ \\
\hline ISLAND & $\begin{array}{l}\text { VITAL } \\
\text { RATIO } \\
\text { COMPONENT II }\end{array}$ & $\begin{array}{l}6098 \\
6098 \\
6098\end{array}$ & $\begin{array}{l}6100 \\
6300 \\
6900\end{array}$ & $\begin{array}{l}6800 \\
7000 \\
8200\end{array}$ & $\begin{array}{l}8200 \\
7700 \\
8500\end{array}$ & $\begin{array}{l}8300 \\
7700 \\
8500\end{array}$ & $\begin{array}{l}8900 \\
8800 \\
8200\end{array}$ & $\begin{array}{l}8900 \\
8800 \\
8100\end{array}$ \\
\hline JEFFERSON & $\begin{array}{l}\text { VITAL } \\
\text { RATIO } \\
\text { COMPONENT II }\end{array}$ & $\begin{array}{l}8918 \\
8918 \\
8918\end{array}$ & $\begin{array}{r}9900 \\
8800 \\
10100\end{array}$ & $\begin{array}{r}10100 \\
9000 \\
9500\end{array}$ & $\begin{array}{r}10900 \\
8400 \\
8900\end{array}$ & $\begin{array}{r}10600 \\
8100 \\
8000\end{array}$ & $\begin{array}{l}9900 \\
8900 \\
9200\end{array}$ & $\begin{array}{r}10000 \\
9000 \\
9200\end{array}$ \\
\hline KING & $\begin{array}{l}\text { VITAL } \\
\text { RATIO } \\
\text { COMPONENT II }\end{array}$ & $\begin{array}{l}504980 \\
504980 \\
504980\end{array}$ & $\begin{array}{l}547900 \\
533600 \\
602200\end{array}$ & $\begin{array}{l}634500 \\
579000 \\
647500\end{array}$ & $\begin{array}{l}676300 \\
644100 \\
674900\end{array}$ & $\begin{array}{l}681200 \\
667200 \\
703000\end{array}$ & $\begin{array}{l}741900 \\
704900 \\
693400\end{array}$ & $\begin{array}{l}761800 \\
726400 \\
659600\end{array}$ \\
\hline KITSAP & $\begin{array}{l}\text { VITAL } \\
\text { RATIO } \\
\text { COMPONENT II }\end{array}$ & $\begin{array}{l}44387 \\
44387 \\
44387\end{array}$ & $\begin{array}{l}56100 \\
54200 \\
65900\end{array}$ & $\begin{array}{l}75700 \\
64700 \\
80800\end{array}$ & $\begin{array}{r}86100 \\
89800 \\
105500\end{array}$ & $\begin{array}{r}91500 \\
94400 \\
119000\end{array}$ & $\begin{array}{l}105600 \\
101600 \\
136400\end{array}$ & $\begin{array}{r}93400 \\
93200 \\
104700\end{array}$ \\
\hline KITTITAS & $\begin{array}{l}\text { VITAL } \\
\text { RATIO } \\
\text { COMPONENT II }\end{array}$ & $\begin{array}{l}20230 \\
20230 \\
20230\end{array}$ & $\begin{array}{l}21800 \\
19800 \\
22100\end{array}$ & $\begin{array}{l}17300 \\
19600 \\
20300\end{array}$ & $\begin{array}{l}17100 \\
19200 \\
21000\end{array}$ & $\begin{array}{l}21100 \\
19400 \\
20300\end{array}$ & $\begin{array}{l}20400 \\
20300 \\
20900\end{array}$ & $\begin{array}{l}22800 \\
21300 \\
20900\end{array}$ \\
\hline KLICKITAT & $\begin{array}{l}\text { VITAL } \\
\text { RATIO } \\
\text { COMPONENT II }\end{array}$ & $\begin{array}{l}11357 \\
11357 \\
11357\end{array}$ & $\begin{array}{l}10900 \\
10600 \\
12400\end{array}$ & $\begin{array}{r}8500 \\
10100 \\
10700\end{array}$ & $\begin{array}{r}8700 \\
9600 \\
10800\end{array}$ & $\begin{array}{r}9200 \\
9500 \\
10800\end{array}$ & $\begin{array}{l}10400 \\
11200 \\
10400\end{array}$ & $\begin{array}{r}9300 \\
12000 \\
10200\end{array}$ \\
\hline LEWIS & $\begin{array}{l}\text { VITAL } \\
\text { RATIO } \\
\text { COMPONENT II }\end{array}$ & $\begin{array}{l}41393 \\
41393 \\
41393\end{array}$ & $\begin{array}{l}38600 \\
40500 \\
45400\end{array}$ & $\begin{array}{l}41100 \\
40800 \\
43500\end{array}$ & $\begin{array}{l}37300 \\
38900 \\
42800\end{array}$ & $\begin{array}{l}40300 \\
39800 \\
42000\end{array}$ & $\begin{array}{l}42400 \\
41600 \\
42700\end{array}$ & $\begin{array}{l}43800 \\
44800 \\
42800\end{array}$ \\
\hline LINCOLN & $\begin{array}{l}\text { VITAL } \\
\text { RATIO } \\
\text { COMPONENT I I }\end{array}$ & $\begin{array}{l}11361 \\
11361 \\
11361\end{array}$ & $\begin{array}{l}11200 \\
11100 \\
11700\end{array}$ & $\begin{array}{r}9000 \\
11000 \\
11100\end{array}$ & $\begin{array}{r}11500 \\
9900 \\
10100\end{array}$ & $\begin{array}{r}11900 \\
10000 \\
9800\end{array}$ & $\begin{array}{r}10900 \\
10300 \\
9500\end{array}$ & $\begin{array}{r}10900 \\
11100 \\
9900\end{array}$ \\
\hline MASON & $\begin{array}{l}\text { VITAL } \\
\text { RATIO } \\
\text { COMPONENT II }\end{array}$ & $\begin{array}{l}11603 \\
11603 \\
11603\end{array}$ & $\begin{array}{l}10500 \\
11900 \\
13600\end{array}$ & $\begin{array}{l}12400 \\
12700 \\
14600\end{array}$ & $\begin{array}{l}13100 \\
12000 \\
12300\end{array}$ & $\begin{array}{l}11900 \\
12000 \\
12100\end{array}$ & $\begin{array}{l}11400 \\
13500 \\
13900\end{array}$ & $\begin{array}{l}12700 \\
14100 \\
13500\end{array}$ \\
\hline
\end{tabular}


TABLE 4a. (contd)

\begin{tabular}{|c|c|c|c|c|c|c|c|c|}
\hline & & $1940^{(b)}$ & 1941 & 1942 & 1943 & 1944 & 1945 & 1946 \\
\hline ANOGAN & $\begin{array}{l}\text { VITAL } \\
\text { RATI0 } \\
\text { COMPONENT II }\end{array}$ & $\begin{array}{l}24546 \\
24546 \\
24546\end{array}$ & $\begin{array}{l}21400 \\
23800 \\
25700\end{array}$ & $\begin{array}{l}20200 \\
24000 \\
25600\end{array}$ & $\begin{array}{l}21700 \\
23200 \\
23000\end{array}$ & $\begin{array}{l}20600 \\
24100 \\
24500\end{array}$ & $\begin{array}{l}22100 \\
25400 \\
25500\end{array}$ & $\begin{array}{l}26500 \\
27800 \\
27600\end{array}$ \\
\hline DACIFIC & $\begin{array}{l}\text { VITAL } \\
\text { RATIO } \\
\text { COMPONENT II }\end{array}$ & $\begin{array}{l}15928 \\
15928 \\
15928\end{array}$ & $\begin{array}{l}14200 \\
15700 \\
17400\end{array}$ & $\begin{array}{l}14500 \\
15000 \\
14600\end{array}$ & $\begin{array}{l}13300 \\
13500 \\
14500\end{array}$ & $\begin{array}{l}14400 \\
14100 \\
15200\end{array}$ & $\begin{array}{l}15800 \\
16000 \\
15200\end{array}$ & $\begin{array}{l}14800 \\
17500 \\
16200\end{array}$ \\
\hline $\begin{array}{l}\text { END } \\
\text { ORIELLE }\end{array}$ & $\begin{array}{l}\text { VITAL } \\
\text { RATIO } \\
\text { COMPONENT II }\end{array}$ & $\begin{array}{l}7156 \\
7156 \\
7156\end{array}$ & $\begin{array}{l}6500 \\
6600 \\
7500\end{array}$ & $\begin{array}{l}5700 \\
6700 \\
7400\end{array}$ & $\begin{array}{l}6200 \\
6100 \\
6700\end{array}$ & $\begin{array}{l}5400 \\
6000 \\
6200\end{array}$ & $\begin{array}{l}5200 \\
6400 \\
6100\end{array}$ & $\begin{array}{l}5300 \\
7200 \\
6700\end{array}$ \\
\hline ERCE & $\begin{array}{l}\text { VITAL } \\
\text { RATIO } \\
\text { COMPONENT II }\end{array}$ & $\begin{array}{l}182081 \\
182081 \\
182081\end{array}$ & $\begin{array}{l}195600 \\
200400 \\
213800\end{array}$ & $\begin{array}{l}214100 \\
215100 \\
236500\end{array}$ & $\begin{array}{l}240200 \\
230700 \\
244100\end{array}$ & $\begin{array}{l}230300 \\
232000 \\
255700\end{array}$ & $\begin{array}{l}241700 \\
247000 \\
262700\end{array}$ & $\begin{array}{l}242000 \\
254800 \\
251700\end{array}$ \\
\hline N JUAN & $\begin{array}{l}\text { VITAL } \\
\text { RATI0 } \\
\text { COMPONENT II }\end{array}$ & $\begin{array}{l}3157 \\
3157 \\
3157\end{array}$ & $\begin{array}{l}2400 \\
2900 \\
3100\end{array}$ & $\begin{array}{l}2800 \\
2800 \\
2800\end{array}$ & $\begin{array}{l}2600 \\
2500 \\
2400\end{array}$ & $\begin{array}{l}2100 \\
2500 \\
2100\end{array}$ & $\begin{array}{l}3200 \\
2700 \\
2000\end{array}$ & $\begin{array}{l}3800 \\
3000 \\
2400\end{array}$ \\
\hline$A G]$ & $\begin{array}{l}\text { VITAL } \\
\text { RATIO } \\
\text { COMPONENT II }\end{array}$ & $\begin{array}{l}37650 \\
37650 \\
37650\end{array}$ & $\begin{array}{l}38900 \\
37500 \\
41000\end{array}$ & $\begin{array}{l}38900 \\
38700 \\
41900\end{array}$ & $\begin{array}{l}41900 \\
37900 \\
40700\end{array}$ & $\begin{array}{l}46300 \\
39600 \\
40100\end{array}$ & $\begin{array}{l}45000 \\
41200 \\
42700\end{array}$ & $\begin{array}{l}49000 \\
43000 \\
41700\end{array}$ \\
\hline AMANIA & $\begin{array}{l}\text { VITAL } \\
\text { RATIO } \\
\text { COMPONENT II }\end{array}$ & $\begin{array}{l}4633 \\
4633 \\
4633\end{array}$ & $\begin{array}{l}4700 \\
4700 \\
5200\end{array}$ & $\begin{array}{l}4900 \\
4800 \\
5000\end{array}$ & $\begin{array}{l}5100 \\
3800 \\
4100\end{array}$ & $\begin{array}{l}3300 \\
3800 \\
3300\end{array}$ & $\begin{array}{l}4400 \\
3900 \\
3600\end{array}$ & $\begin{array}{l}4500 \\
4500 \\
3800\end{array}$ \\
\hline OHOMISH & $\begin{array}{l}\text { VITAL } \\
\text { RATIO } \\
\text { COMPONENT II }\end{array}$ & $\begin{array}{l}88754 \\
88754 \\
88754\end{array}$ & $\begin{array}{r}87200 \\
91700 \\
100600\end{array}$ & $\begin{array}{r}88700 \\
95800 \\
103300\end{array}$ & $\begin{array}{r}91700 \\
97600 \\
106000\end{array}$ & $\begin{array}{r}93800 \\
99300 \\
103900\end{array}$ & $\begin{array}{r}99400 \\
106900 \\
99700\end{array}$ & $\begin{array}{r}104700 \\
111400 \\
98000\end{array}$ \\
\hline OKANE & $\begin{array}{l}\text { VITAL } \\
\text { RATIO } \\
\text { COMPONENT II }\end{array}$ & $\begin{array}{l}164652 \\
164652 \\
164652\end{array}$ & $\begin{array}{l}163300 \\
165600 \\
180000\end{array}$ & $\begin{array}{l}168800 \\
181700 \\
208600\end{array}$ & $\begin{array}{l}182300 \\
188600 \\
210200\end{array}$ & $\begin{array}{l}191200 \\
194200 \\
210800\end{array}$ & $\begin{array}{l}201100 \\
201500 \\
204000\end{array}$ & $\begin{array}{l}216900 \\
209700 \\
192500\end{array}$ \\
\hline EVENS & $\begin{array}{l}\text { VITAL } \\
\text { RATIO } \\
\text { COMPONENT II }\end{array}$ & $\begin{array}{l}19275 \\
19275 \\
19275\end{array}$ & $\begin{array}{l}17500 \\
18100 \\
19000\end{array}$ & $\begin{array}{l}16000 \\
17400 \\
17300\end{array}$ & $\begin{array}{l}15400 \\
16000 \\
15700\end{array}$ & $\begin{array}{l}14400 \\
16000 \\
14700\end{array}$ & $\begin{array}{l}14200 \\
15800 \\
17300\end{array}$ & $\begin{array}{l}15000 \\
17100 \\
18200\end{array}$ \\
\hline JRST & $\begin{array}{l}\text { VITAL } \\
\text { RATIO } \\
\text { COMPONENT II }\end{array}$ & $\begin{array}{l}37285 \\
37285 \\
37285\end{array}$ & $\begin{array}{l}41300 \\
38500 \\
42500\end{array}$ & $\begin{array}{l}39000 \\
39500 \\
43300\end{array}$ & $\begin{array}{l}44200 \\
39600 \\
41200\end{array}$ & $\begin{array}{l}40200 \\
38900 \\
40100\end{array}$ & $\begin{array}{l}40700 \\
41000 \\
41300\end{array}$ & $\begin{array}{l}44000 \\
42700 \\
39900\end{array}$ \\
\hline IKIAK & $\begin{array}{l}\text { VITAL } \\
\text { RATIO } \\
\text { COMPONENT II }\end{array}$ & $\begin{array}{l}4286 \\
4286 \\
4286\end{array}$ & $\begin{array}{l}3600 \\
4500 \\
4400\end{array}$ & $\begin{array}{l}4200 \\
4200 \\
4000\end{array}$ & $\begin{array}{l}3500 \\
3800 \\
3700\end{array}$ & $\begin{array}{l}3400 \\
3500 \\
2500\end{array}$ & $\begin{array}{l}3300 \\
3900 \\
4000\end{array}$ & $\begin{array}{l}3800 \\
4200 \\
3900\end{array}$ \\
\hline
\end{tabular}


TABLE 4a. (contd)

\begin{tabular}{|c|c|c|c|c|c|c|c|c|}
\hline & & $1940^{(b)}$ & 1941 & 1942 & 1943 & 1944 & 1945 & 1946 \\
\hline $\begin{array}{l}\text { WALLA } \\
\text { WALLA }\end{array}$ & $\begin{array}{l}\text { VITAL } \\
\text { RATIO } \\
\text { COMPONENT II }\end{array}$ & $\begin{array}{l}30547 \\
30547 \\
30547\end{array}$ & $\begin{array}{l}31100 \\
30200 \\
32600\end{array}$ & $\begin{array}{l}29600 \\
31100 \\
34300\end{array}$ & $\begin{array}{l}31600 \\
32000 \\
34300\end{array}$ & $\begin{array}{l}36600 \\
32100 \\
31800\end{array}$ & $\begin{array}{l}39600 \\
35500 \\
33800\end{array}$ & $\begin{array}{l}40500 \\
36600 \\
33800\end{array}$ \\
\hline WHATCOM & $\begin{array}{l}\text { VITAL } \\
\text { RATIO } \\
\text { COMPONENT II }\end{array}$ & $\begin{array}{l}60355 \\
60355 \\
60355\end{array}$ & $\begin{array}{l}59100 \\
59800 \\
64500\end{array}$ & $\begin{array}{l}61200 \\
60500 \\
61300\end{array}$ & $\begin{array}{l}64400 \\
59100 \\
60000\end{array}$ & $\begin{array}{l}65000 \\
60400 \\
59600\end{array}$ & $\begin{array}{l}66600 \\
62800 \\
57100\end{array}$ & $\begin{array}{l}71700 \\
66400 \\
56700\end{array}$ \\
\hline WHITMAN & $\begin{array}{l}\text { VITAL } \\
\text { RATIO } \\
\text { COMPONENT II }\end{array}$ & $\begin{array}{l}27221 \\
27221 \\
27221\end{array}$ & $\begin{array}{l}26800 \\
26400 \\
29400\end{array}$ & $\begin{array}{l}26400 \\
26500 \\
26900\end{array}$ & $\begin{array}{l}24700 \\
24100 \\
26000\end{array}$ & $\begin{array}{l}27400 \\
25600 \\
26700\end{array}$ & $\begin{array}{l}25300 \\
26500 \\
25600\end{array}$ & $\begin{array}{l}29000 \\
28200 \\
25300\end{array}$ \\
\hline YAKIMA & $\begin{array}{l}\text { VITAL } \\
\text { RATIO } \\
\text { COMPONENT II }\end{array}$ & $\begin{array}{l}99019 \\
99019 \\
99019\end{array}$ & $\begin{array}{r}96000 \\
96500 \\
104300\end{array}$ & $\begin{array}{r}88300 \\
96100 \\
100100\end{array}$ & $\begin{array}{r}91900 \\
96000 \\
106400\end{array}$ & $\begin{array}{l}104400 \\
101500 \\
111100\end{array}$ & $\begin{array}{l}113200 \\
112100 \\
125700\end{array}$ & $\begin{array}{l}120100 \\
121400 \\
128300\end{array}$ \\
\hline
\end{tabular}

\footnotetext{
(a) Intercensus estimates are rounded to nearest hundred.

(b) Actual census count.

(c) Average of birth and death estimates.
} 
TABLE 4b. Population Estimates for Washington Counties $1947-1953^{(a)}$

\begin{tabular}{|c|c|c|c|c|c|c|c|c|}
\hline & & 1947 & 1948 & 1949 & $1950^{(b)}$ & 1951 & 1952 & 1953 \\
\hline ADAMS & $\begin{array}{l}\text { VITAL } \\
\text { RATIO } \\
\text { COMPONENT II }\end{array}$ & $\begin{array}{l}6700 \\
6100 \\
5400\end{array}$ & $\begin{array}{l}8200 \\
6400 \\
5800\end{array}$ & $\begin{array}{l}8500 \\
6500 \\
5800\end{array}$ & $\begin{array}{l}6584 \\
6584 \\
6584\end{array}$ & $\begin{array}{l}5700 \\
6500 \\
7300\end{array}$ & $\begin{array}{l}7100 \\
6900 \\
7800\end{array}$ & $\begin{array}{l}6600 \\
7300 \\
8400\end{array}$ \\
\hline ASOTIN & $\begin{array}{l}\text { VITAL } \\
\text { RATIO } \\
\text { COMPONENT II }\end{array}$ & $\begin{array}{r}10000 \\
9300 \\
7700\end{array}$ & $\begin{array}{l}7600 \\
8900 \\
8800\end{array}$ & $\begin{array}{r}12400 \\
10100 \\
9300\end{array}$ & $\begin{array}{l}10878 \\
10878 \\
10878\end{array}$ & $\begin{array}{l}12000 \\
11200 \\
11400\end{array}$ & $\begin{array}{l}10800 \\
10900 \\
11400\end{array}$ & $\begin{array}{l}11100 \\
11600 \\
12000\end{array}$ \\
\hline BENTON & $\begin{array}{l}\text { VITAL } \\
\text { RATIO } \\
\text { COMPONENT I I }\end{array}$ & $\begin{array}{l}29096 \\
42100 \\
31996\end{array}$ & $\begin{array}{l}54428 \\
62400 \\
71928\end{array}$ & $\begin{array}{l}56248 \\
57900 \\
53348\end{array}$ & $\begin{array}{l}57267 \\
57267 \\
57267\end{array}$ & $\begin{array}{l}60000 \\
58300 \\
57200\end{array}$ & $\begin{array}{l}66200 \\
59900 \\
59400\end{array}$ & $\begin{array}{l}60900 \\
62600 \\
66200\end{array}$ \\
\hline CHELAN & $\begin{array}{l}\text { VITAL } \\
\text { RATIO } \\
\text { COMPONENT II }\end{array}$ & $\begin{array}{l}43000 \\
38800 \\
39800\end{array}$ & $\begin{array}{l}43300 \\
38300 \\
43300\end{array}$ & $\begin{array}{l}42500 \\
38500 \\
40300\end{array}$ & $\begin{array}{l}39301 \\
39301 \\
39301\end{array}$ & $\begin{array}{l}34900 \\
38400 \\
42500\end{array}$ & $\begin{array}{l}36500 \\
39100 \\
44000\end{array}$ & $\begin{array}{l}35500 \\
39800 \\
46100\end{array}$ \\
\hline CLALLAM & $\begin{array}{l}\text { VITAL } \\
\text { RATIO } \\
\text { COMPONENT II }\end{array}$ & $\begin{array}{l}24600 \\
23900 \\
22900\end{array}$ & $\begin{array}{l}26400 \\
24600 \\
26800\end{array}$ & $\begin{array}{l}23900 \\
25400 \\
26300\end{array}$ & $\begin{array}{l}26396 \\
26396 \\
26396\end{array}$ & $\begin{array}{l}29400 \\
26600 \\
28900\end{array}$ & $\begin{array}{l}27900 \\
27100 \\
30900\end{array}$ & $\begin{array}{l}29900 \\
27200 \\
31400\end{array}$ \\
\hline CLARK & $\begin{array}{l}\text { VITAL } \\
\text { RATIO } \\
\text { COMPONENT II }\end{array}$ & $\begin{array}{l}86600 \\
84900 \\
91500\end{array}$ & $\begin{array}{r}84900 \\
86000 \\
108500\end{array}$ & $\begin{array}{l}85700 \\
86900 \\
96800\end{array}$ & $\begin{array}{l}85307 \\
85307 \\
85307\end{array}$ & $\begin{array}{l}88700 \\
87300 \\
94200\end{array}$ & $\begin{array}{l}86700 \\
86800 \\
91900\end{array}$ & $\begin{array}{l}84100 \\
85100 \\
92600\end{array}$ \\
\hline COLUMBIA & $\begin{array}{l}\text { VITAL } \\
\text { RATIO } \\
\text { COMPONENT II }\end{array}$ & $\begin{array}{l}5800 \\
4900 \\
4600\end{array}$ & $\begin{array}{l}6600 \\
4800 \\
5100\end{array}$ & $\begin{array}{l}5600 \\
4900 \\
5200\end{array}$ & $\begin{array}{l}4860 \\
4860 \\
4860\end{array}$ & $\begin{array}{l}4300 \\
4400 \\
4900\end{array}$ & $\begin{array}{l}5500 \\
4600 \\
4800\end{array}$ & $\begin{array}{l}4400 \\
4200 \\
4700\end{array}$ \\
\hline COWLITZ & $\begin{array}{l}\text { VITAL } \\
\text { RATIO } \\
\text { COMPONENT II }\end{array}$ & $\begin{array}{l}48200 \\
46600 \\
46500\end{array}$ & $\begin{array}{l}52100 \\
48300 \\
54400\end{array}$ & $\begin{array}{l}51200 \\
49300 \\
54500\end{array}$ & $\begin{array}{l}53369 \\
53369 \\
53369\end{array}$ & $\begin{array}{l}55300 \\
55600 \\
58800\end{array}$ & $\begin{array}{l}57300 \\
55800 \\
58100\end{array}$ & $\begin{array}{l}55700 \\
56700 \\
58200\end{array}$ \\
\hline DOUGLAS & $\begin{array}{l}\text { VITAL } \\
\text { RATIO } \\
\text { COMPONENT II }\end{array}$ & $\begin{array}{l}8600 \\
9200 \\
6800\end{array}$ & $\begin{array}{l}9600 \\
9400 \\
7800\end{array}$ & $\begin{array}{r}10900 \\
10300 \\
8500\end{array}$ & $\begin{array}{l}10817 \\
10817 \\
10817\end{array}$ & $\begin{array}{l}11200 \\
11500 \\
12600\end{array}$ & $\begin{array}{l}14500 \\
11900 \\
12900\end{array}$ & $\begin{array}{l}13200 \\
12900 \\
12700\end{array}$ \\
\hline FERRY & $\begin{array}{l}\text { VITAL } \\
\text { RATIO } \\
\text { COMPONENT II }\end{array}$ & $\begin{array}{l}2800 \\
3800 \\
3500\end{array}$ & $\begin{array}{l}4100 \\
3900 \\
3800\end{array}$ & $\begin{array}{l}3800 \\
3900 \\
3600\end{array}$ & $\begin{array}{l}4096 \\
4096 \\
4096\end{array}$ & $\begin{array}{l}4500 \\
3700 \\
4200\end{array}$ & $\begin{array}{l}5200 \\
3800 \\
4300\end{array}$ & $\begin{array}{l}4300 \\
3800 \\
4300\end{array}$ \\
\hline FRANKLIN & $\begin{array}{l}\text { VITAL } \\
\text { RATIO } \\
\text { COMPONENT II }\end{array}$ & $\begin{array}{l}10500 \\
11300 \\
11200\end{array}$ & $\begin{array}{l}11400 \\
11900 \\
14300\end{array}$ & $\begin{array}{l}13000 \\
12900 \\
12800\end{array}$ & $\begin{array}{l}13563 \\
13563 \\
13563\end{array}$ & $\begin{array}{l}14300 \\
14800 \\
15200\end{array}$ & $\begin{array}{l}17800 \\
15300 \\
15900\end{array}$ & $\begin{array}{l}17200 \\
16400 \\
18200\end{array}$ \\
\hline GARF IELD & $\begin{array}{l}\text { VITAL } \\
\text { RATIO } \\
\text { COMPONENT II }\end{array}$ & $\begin{array}{l}2800 \\
3000 \\
2800\end{array}$ & $\begin{array}{l}3100 \\
3100 \\
3200\end{array}$ & $\begin{array}{l}3400 \\
3400 \\
3000\end{array}$ & $\begin{array}{l}3204 \\
3204 \\
3204\end{array}$ & $\begin{array}{l}3600 \\
3000 \\
3300\end{array}$ & $\begin{array}{l}3100 \\
2800 \\
3300\end{array}$ & $\begin{array}{l}3500 \\
2900 \\
3400\end{array}$ \\
\hline
\end{tabular}


TABLE 4b. (contd)

\begin{tabular}{|c|c|c|c|c|c|c|c|c|}
\hline GRANT & $\begin{array}{l}\text { VITAL } \\
\text { RATIO } \\
\text { COMPONENT II }\end{array}$ & $\begin{array}{l}14900 \\
16500 \\
12800\end{array}$ & $\begin{array}{l}14800 \\
17000 \\
15100\end{array}$ & $\begin{array}{l}17000 \\
21300 \\
20600\end{array}$ & $\begin{array}{l}24346 \\
24346 \\
24346\end{array}$ & $\begin{array}{l}24300 \\
26700 \\
26800\end{array}$ & $\begin{array}{l}23900 \\
27200 \\
28200\end{array}$ & $\begin{array}{l}26600 \\
30200 \\
30300\end{array}$ \\
\hline $\begin{array}{l}\text { GRAYS } \\
\text { HARBOR }\end{array}$ & $\begin{array}{l}\text { VITAL } \\
\text { RATI0 } \\
\text { COMPONENT } 11\end{array}$ & $\begin{array}{l}56400 \\
54000 \\
51500\end{array}$ & $\begin{array}{l}61200 \\
54600 \\
57800\end{array}$ & $\begin{array}{l}57500 \\
54300 \\
54200\end{array}$ & $\begin{array}{l}53644 \\
53644 \\
53644\end{array}$ & $\begin{array}{l}54000 \\
52300 \\
55700\end{array}$ & $\begin{array}{l}49800 \\
52000 \\
55300\end{array}$ & $\begin{array}{l}48000 \\
50700 \\
55800\end{array}$ \\
\hline ISLAND & $\begin{array}{l}\text { VITAL } \\
\text { RATIO } \\
\text { COMPONENT . II }\end{array}$ & $\begin{array}{l}9000 \\
8400 \\
8300\end{array}$ & $\begin{array}{r}8700 \\
8700 \\
10800\end{array}$ & $\begin{array}{r}9000 \\
9300 \\
11500\end{array}$ & $\begin{array}{l}11079 \\
11079 \\
11079\end{array}$ & $\begin{array}{l}12400 \\
11500 \\
13000\end{array}$ & $\begin{array}{l}12100 \\
11700 \\
13500\end{array}$ & $\begin{array}{l}16600 \\
13800 \\
14300\end{array}$ \\
\hline JEFFERSON & $\begin{array}{l}\text { VITAL } \\
\text { RATIO } \\
\text { COMPONENT II }\end{array}$ & $\begin{array}{l}9500 \\
8700 \\
9200\end{array}$ & $\begin{array}{r}10500 \\
9400 \\
11300\end{array}$ & $\begin{array}{l}12900 \\
10000 \\
11700\end{array}$ & $\begin{array}{l}11618 \\
11618 \\
11618\end{array}$ & $\begin{array}{l}10600 \\
11600 \\
13000\end{array}$ & $\begin{array}{l}12200 \\
12000 \\
12500\end{array}$ & $\begin{array}{r}9900 \\
11100 \\
10800\end{array}$ \\
\hline KING & $\begin{array}{l}\text { VITAL } \\
\text { RATIO } \\
\text { COMPONENT II }\end{array}$ & $\begin{array}{l}724100 \\
695900 \\
662500\end{array}$ & $\begin{array}{l}740500 \\
707700 \\
777500\end{array}$ & $\begin{array}{l}750700 \\
716900 \\
767400\end{array}$ & $\begin{array}{l}732992 \\
732992 \\
732992\end{array}$ & $\begin{array}{l}746000 \\
760000 \\
794200\end{array}$ & $\begin{array}{l}755900 \\
771200 \\
802200\end{array}$ & $\begin{array}{l}764600 \\
772900 \\
825100\end{array}$ \\
\hline KITSAP & $\begin{array}{l}\text { VITAL } \\
\text { RATIO } \\
\text { COMPONENT II }\end{array}$ & $\begin{array}{l}74500 \\
79700 \\
93000\end{array}$ & $\begin{array}{r}70500 \\
78300 \\
102200\end{array}$ & $\begin{array}{l}69200 \\
71700 \\
86500\end{array}$ & $\begin{array}{l}75724 \\
75724 \\
75724\end{array}$ & $\begin{array}{l}87500 \\
79100 \\
84300\end{array}$ & $\begin{array}{l}91000 \\
80800 \\
88400\end{array}$ & $\begin{array}{l}86600 \\
79600 \\
86700\end{array}$ \\
\hline KITTITAS & $\begin{array}{l}\text { VITAL } \\
\text { RATIO } \\
\text { COMPONENT II }\end{array}$ & $\begin{array}{l}21100 \\
21000 \\
21300\end{array}$ & $\begin{array}{l}23200 \\
21100 \\
23400\end{array}$ & $\begin{array}{l}22800 \\
21800 \\
23500\end{array}$ & $\begin{array}{l}22235 \\
22235 \\
22235\end{array}$ & $\begin{array}{l}21200 \\
20700 \\
23200\end{array}$ & $\begin{array}{l}19900 \\
20100 \\
21900\end{array}$ & $\begin{array}{l}20100 \\
20100 \\
22200\end{array}$ \\
\hline KLICKITAT & $\begin{array}{l}\text { VITAL } \\
\text { RATIO } \\
\text { COMPONENT II }\end{array}$ & $\begin{array}{l}10300 \\
11300 \\
10500\end{array}$ & $\begin{array}{l}12100 \\
11700 \\
12100\end{array}$ & $\begin{array}{l}12800 \\
12200 \\
12000\end{array}$ & $\begin{array}{l}12049 \\
12049 \\
12049\end{array}$ & $\begin{array}{l}11400 \\
12000 \\
13900\end{array}$ & $\begin{array}{l}12400 \\
12100 \\
14500\end{array}$ & $\begin{array}{l}12200 \\
12500 \\
16100\end{array}$ \\
\hline LEWIS & $\begin{array}{l}\text { VITAL } \\
\text { RATIO } \\
\text { COMPONENT II }\end{array}$ & $\begin{array}{l}40500 \\
43100 \\
43100\end{array}$ & $\begin{array}{l}44400 \\
43100 \\
48300\end{array}$ & $\begin{array}{l}44100 \\
43800 \\
44900\end{array}$ & $\begin{array}{l}43755 \\
43755 \\
43755\end{array}$ & $\begin{array}{l}42500 \\
42000 \\
46200\end{array}$ & $\begin{array}{l}40900 \\
41900 \\
46100\end{array}$ & $\begin{array}{l}39700 \\
41300 \\
46900\end{array}$ \\
\hline LINCOLN & $\begin{array}{l}\text { VITAL } \\
\text { RATIO } \\
\text { COMPONENT II }\end{array}$ & $\begin{array}{r}12800 \\
10100 \\
9800\end{array}$ & $\begin{array}{l}10300 \\
10300 \\
10800\end{array}$ & $\begin{array}{l}11500 \\
10900 \\
10600\end{array}$ & $\begin{array}{l}10970 \\
10970 \\
10970\end{array}$ & $\begin{array}{l}10200 \\
10400 \\
11600\end{array}$ & $\begin{array}{l}12400 \\
10600 \\
11100\end{array}$ & $\begin{array}{l}10100 \\
10500 \\
11300\end{array}$ \\
\hline MASON & $\begin{array}{l}\text { VITAL } \\
\text { RATIO } \\
\text { COMPONENT II }\end{array}$ & $\begin{array}{l}12700 \\
13700 \\
13300\end{array}$ & $\begin{array}{l}13300 \\
13800 \\
14800\end{array}$ & $\begin{array}{l}13100 \\
14400 \\
15500\end{array}$ & $\begin{array}{l}15022 \\
15022 \\
15022\end{array}$ & $\begin{array}{l}17100 \\
15100 \\
16600\end{array}$ & $\begin{array}{l}17800 \\
15300 \\
16700\end{array}$ & $\begin{array}{l}18300 \\
15200 \\
17000\end{array}$ \\
\hline OKANOGAN & $\begin{array}{l}\text { VITAL } \\
\text { RATIO } \\
\text { COMPONENT II }\end{array}$ & $\begin{array}{l}24800 \\
27100 \\
27400\end{array}$ & $\begin{array}{l}29100 \\
26900 \\
29100\end{array}$ & $\begin{array}{l}26700 \\
27400 \\
28800\end{array}$ & $\begin{array}{l}29131 \\
29131 \\
29131\end{array}$ & $\begin{array}{l}25400 \\
26900 \\
28800\end{array}$ & $\begin{array}{l}22600 \\
26400 \\
27900\end{array}$ & $\begin{array}{l}22700 \\
26400 \\
28800\end{array}$ \\
\hline
\end{tabular}


TABLE 4b. (contd)

\begin{tabular}{|c|c|c|c|c|c|c|c|c|}
\hline PACIFIC & $\begin{array}{l}\text { VITAL } \\
\text { RATIO } \\
\text { COMPONENT II }\end{array}$ & $\begin{array}{l}15000 \\
17100 \\
16000\end{array}$ & $\begin{array}{l}18100 \\
17300 \\
18000\end{array}$ & $\begin{array}{l}15700 \\
17300 \\
16900\end{array}$ & $\begin{array}{l}16558 \\
16558 \\
16558\end{array}$ & $\begin{array}{l}16400 \\
16000 \\
17700\end{array}$ & $\begin{array}{l}18100 \\
16100 \\
17700\end{array}$ & $\begin{array}{l}16700 \\
15600 \\
17000\end{array}$ \\
\hline $\begin{array}{l}\text { PEND } \\
\text { ORIELLE }\end{array}$ & $\begin{array}{l}\text { VITAL } \\
\text { RATIO } \\
\text { COMPONENT II }\end{array}$ & $\begin{array}{l}6300 \\
6900 \\
6400\end{array}$ & $\begin{array}{l}6300 \\
6800 \\
6900\end{array}$ & $\begin{array}{l}6800 \\
7300 \\
7400\end{array}$ & $\begin{array}{l}7413 \\
7413 \\
7413\end{array}$ & $\begin{array}{l}8900 \\
7100 \\
7800\end{array}$ & $\begin{array}{l}8900 \\
7300 \\
8500\end{array}$ & $\begin{array}{l}9300 \\
7600 \\
8600\end{array}$ \\
\hline PIERCE & $\begin{array}{l}\text { VITAL } \\
\text { RATIO } \\
\text { COMPONENT II }\end{array}$ & $\begin{array}{l}232000 \\
240900 \\
250400\end{array}$ & $\begin{array}{l}222400 \\
245800 \\
288600\end{array}$ & $\begin{array}{l}230000 \\
253100 \\
285100\end{array}$ & $\begin{array}{l}275876 \\
275876 \\
275876\end{array}$ & $\begin{array}{l}274100 \\
280100 \\
294500\end{array}$ & $\begin{array}{l}278400 \\
283100 \\
290200\end{array}$ & $\begin{array}{l}283100 \\
287600 \\
297100\end{array}$ \\
\hline SAN JUAN & $\begin{array}{l}\text { VITAL } \\
\text { RATIO } \\
\text { COMPONENT II }\end{array}$ & $\begin{array}{l}3400 \\
3000 \\
2500\end{array}$ & $\begin{array}{l}3700 \\
3000 \\
3000\end{array}$ & $\begin{array}{l}3100 \\
3200 \\
2900\end{array}$ & $\begin{array}{l}3245 \\
3245 \\
3245\end{array}$ & $\begin{array}{l}3500 \\
3100 \\
3500\end{array}$ & $\begin{array}{l}2600 \\
3000 \\
3300\end{array}$ & $\begin{array}{l}3200 \\
3000 \\
3300\end{array}$ \\
\hline SKAGIT & $\begin{array}{l}\text { VITAL } \\
\text { RATIO } \\
\text { COMPONENT II }\end{array}$ & $\begin{array}{l}47400 \\
41500 \\
41600\end{array}$ & $\begin{array}{l}34900 \\
42200 \\
46300\end{array}$ & $\begin{array}{l}36900 \\
42300 \\
45200\end{array}$ & $\begin{array}{l}43273 \\
43273 \\
43273\end{array}$ & $\begin{array}{l}45600 \\
42000 \\
45500\end{array}$ & $\begin{array}{l}40800 \\
42000 \\
46000\end{array}$ & $\begin{array}{l}42300 \\
42200 \\
47400\end{array}$ \\
\hline SKAMANIA & $\begin{array}{l}\text { VITAL } \\
\text { RATIO } \\
\text { COMPONENT II }\end{array}$ & $\begin{array}{l}5500 \\
4200 \\
4000\end{array}$ & $\begin{array}{l}6000 \\
4200 \\
4300\end{array}$ & $\begin{array}{l}5100 \\
4500 \\
4300\end{array}$ & $\begin{array}{l}4788 \\
4788 \\
4788\end{array}$ & $\begin{array}{l}4600 \\
4500 \\
5400\end{array}$ & $\begin{array}{l}5600 \\
4500 \\
5200\end{array}$ & $\begin{array}{l}4900 \\
4900 \\
5500\end{array}$ \\
\hline SNOHOMISH & $\begin{array}{l}\text { VITAL } \\
\text { RATIO } \\
\text { COMPONENT II }\end{array}$ & $\begin{array}{r}105500 \\
108100 \\
97500\end{array}$ & $\begin{array}{l}112200 \\
110000 \\
111900\end{array}$ & $\begin{array}{l}106200 \\
110200 \\
112100\end{array}$ & $\begin{array}{l}111580 \\
111580 \\
111580\end{array}$ & $\begin{array}{l}115200 \\
116500 \\
122000\end{array}$ & $\begin{array}{l}114900 \\
117400 \\
122600\end{array}$ & $\begin{array}{l}121700 \\
120500 \\
127300\end{array}$ \\
\hline SPOKANE & $\begin{array}{l}\text { VITAL } \\
\text { RATIO } \\
\text { COMPONENT II }\end{array}$ & $\begin{array}{l}209500 \\
205000 \\
193400\end{array}$ & $\begin{array}{l}211100 \\
211800 \\
223700\end{array}$ & $\begin{array}{l}219000 \\
215900 \\
224000\end{array}$ & $\begin{array}{l}221561 \\
221561 \\
221561\end{array}$ & $\begin{array}{l}227400 \\
229000 \\
247600\end{array}$ & $\begin{array}{l}236900 \\
233700 \\
252600\end{array}$ & $\begin{array}{l}247500 \\
236300 \\
258900\end{array}$ \\
\hline STEVENS & $\begin{array}{l}\text { VITAL } \\
\text { RATIO } \\
\text { COMPONENT II }\end{array}$ & $\begin{array}{l}15600 \\
16700 \\
18300\end{array}$ & $\begin{array}{l}17900 \\
17200 \\
21100\end{array}$ & $\begin{array}{l}17000 \\
17100 \\
19500\end{array}$ & $\begin{array}{l}18580 \\
18580 \\
18580\end{array}$ & $\begin{array}{l}19000 \\
18000 \\
19800\end{array}$ & $\begin{array}{l}18500 \\
17900 \\
19300\end{array}$ & $\begin{array}{l}19100 \\
17800 \\
19300\end{array}$ \\
\hline THURSTON & $\begin{array}{l}\text { VITAL } \\
\text { RATIO } \\
\text { COMPONENT II }\end{array}$ & $\begin{array}{l}41500 \\
41700 \\
39400\end{array}$ & $\begin{array}{l}43800 \\
43000 \\
45800\end{array}$ & $\begin{array}{l}43800 \\
44100 \\
45300\end{array}$ & $\begin{array}{l}44884 \\
44884 \\
44884\end{array}$ & $\begin{array}{l}45600 \\
44700 \\
46200\end{array}$ & $\begin{array}{l}45100 \\
44200 \\
44900\end{array}$ & $\begin{array}{l}42600 \\
44100 \\
46700\end{array}$ \\
\hline WAHKI AKUM & $\begin{array}{l}\text { VITAL } \\
\text { RATIO } \\
\text { COMPONENT II }\end{array}$ & $\begin{array}{l}4100 \\
3700 \\
3500\end{array}$ & $\begin{array}{l}3700 \\
3800 \\
4100\end{array}$ & $\begin{array}{l}4100 \\
3900 \\
4000\end{array}$ & $\begin{array}{l}3835 \\
3835 \\
3835\end{array}$ & $\begin{array}{l}4100 \\
3700 \\
4000\end{array}$ & $\begin{array}{l}4100 \\
3600 \\
3800\end{array}$ & $\begin{array}{l}4200 \\
3600 \\
3900\end{array}$ \\
\hline $\begin{array}{l}\text { WALLA } \\
\text { WALLA }\end{array}$ & $\begin{array}{l}\text { VITAL } \\
\text { RATIO } \\
\text { COMPONENT II }\end{array}$ & $\begin{array}{l}35800 \\
36600 \\
35200\end{array}$ & $\begin{array}{l}37300 \\
37200 \\
40200\end{array}$ & $\begin{array}{l}40900 \\
38200 \\
40200\end{array}$ & $\begin{array}{l}40135 \\
40135 \\
40135\end{array}$ & $\begin{array}{l}39800 \\
40000 \\
42900\end{array}$ & $\begin{array}{l}38000 \\
40100 \\
42900\end{array}$ & $\begin{array}{l}41300 \\
40600 \\
42600\end{array}$ \\
\hline
\end{tabular}


TABLE 4b. (contd)

\begin{tabular}{|c|c|c|c|c|c|c|c|c|}
\hline & & 1947 & 1948 & 1949 & $1950^{(b)}$ & 1951 & 1952 & 1953 \\
\hline WHATCOM & $\begin{array}{l}\text { VITAL } \\
\text { RATIO } \\
\text { COMPONENT II }\end{array}$ & $\begin{array}{l}70700 \\
62400 \\
55400\end{array}$ & $\begin{array}{l}71900 \\
64000 \\
66200\end{array}$ & $\begin{array}{l}71100 \\
65700 \\
66100\end{array}$ & $\begin{array}{l}66733 \\
66733 \\
66733\end{array}$ & $\begin{array}{l}69800 \\
64000 \\
68300\end{array}$ & $\begin{array}{l}62200 \\
63500 \\
68600\end{array}$ & $\begin{array}{l}63200 \\
64500 \\
71500\end{array}$ \\
\hline WHITMAN & $\begin{array}{l}\text { VITAL } \\
\text { RATIO } \\
\text { COMPONENT II }\end{array}$ & $\begin{array}{l}30900 \\
28200 \\
26400\end{array}$ & $\begin{array}{l}32700 \\
28200 \\
29300\end{array}$ & $\begin{array}{l}31300 \\
29500 \\
30000\end{array}$ & $\begin{array}{l}32469 \\
32469 \\
32469\end{array}$ & $\begin{array}{l}31400 \\
31500 \\
33900\end{array}$ & $\begin{array}{l}30700 \\
31000 \\
32500\end{array}$ & $\begin{array}{l}27600 \\
30600 \\
32000\end{array}$ \\
\hline YAKIMA & $\begin{array}{l}\text { VITAL } \\
\text { RATIO } \\
\text { COMPONENT II }\end{array}$ & $\begin{array}{l}116400 \\
124200 \\
129400\end{array}$ & $\begin{array}{l}119800 \\
126200 \\
147600\end{array}$ & $\begin{array}{l}126100 \\
125600 \\
138200\end{array}$ & $\begin{array}{l}135723 \\
135723 \\
135723\end{array}$ & $\begin{array}{l}129000 \\
134400 \\
148400\end{array}$ & $\begin{array}{l}134800 \\
134600 \\
147500\end{array}$ & $\begin{array}{l}134900 \\
132100 \\
156100\end{array}$ \\
\hline
\end{tabular}

(a) Intercensus estimates are rounded to nearest hundred.

(b) Actual census count.

(c) Average of birth and death estimates. 
TABLE 4c. Population Estimates for Washington Counties $1954-1960^{(a)}$

\begin{tabular}{|c|c|c|c|c|c|c|c|c|}
\hline & & 1954 & 1955 & 1956 & 1957 & 1958 & 1959 & $1960^{(\mathrm{b})}$ \\
\hline ADAMS & $\begin{array}{l}\text { VITAL } \\
\text { RATIO } \\
\text { COMPONENT II }\end{array}$ & $\begin{array}{l}6800 \\
7900 \\
9500\end{array}$ & $\begin{array}{l}7600 \\
8646 \\
9500\end{array}$ & $\begin{array}{l}8600 \\
9100 \\
9900\end{array}$ & $\begin{array}{l}8300 \\
9400 \\
8700\end{array}$ & $\begin{array}{r}8300 \\
9600 \\
10000\end{array}$ & $\begin{array}{l}8900 \\
9700 \\
9800\end{array}$ & $\begin{array}{l}9929 \\
9929 \\
9929\end{array}$ \\
\hline ASOTIN & $\begin{array}{l}\text { VITAL } \\
\text { RATIO } \\
\text { COMPONENT II }\end{array}$ & $\begin{array}{l}12700 \\
11800 \\
12400\end{array}$ & $\begin{array}{l}12400 \\
12341 \\
14200\end{array}$ & $\begin{array}{l}12300 \\
12600 \\
14200\end{array}$ & $\begin{array}{l}11900 \\
13000 \\
13200\end{array}$ & $\begin{array}{l}11200 \\
12700 \\
13600\end{array}$ & $\begin{array}{l}11100 \\
12600 \\
12800\end{array}$ & $\begin{array}{l}12909 \\
12909 \\
12909\end{array}$ \\
\hline BENTON & $\begin{array}{l}\text { VITAL } \\
\text { RATIO } \\
\text { COMPONENT II }\end{array}$ & $\begin{array}{l}68200 \\
64000 \\
66500\end{array}$ & $\begin{array}{l}63800 \\
61964 \\
64100\end{array}$ & $\begin{array}{l}60200 \\
61800 \\
63900\end{array}$ & $\begin{array}{l}59300 \\
62000 \\
57200\end{array}$ & $\begin{array}{l}61300 \\
62700 \\
62800\end{array}$ & $\begin{array}{l}61900 \\
62700 \\
64000\end{array}$ & $\begin{array}{l}62070 \\
62070 \\
62070\end{array}$ \\
\hline CHELAN & $\begin{array}{l}\text { VITAL } \\
\text { RATIO } \\
\text { COMPONENT II }\end{array}$ & $\begin{array}{l}38800^{\circ} \\
40700 \\
45900\end{array}$ & $\begin{array}{l}35800 \\
40024 \\
44500\end{array}$ & $\begin{array}{l}36600 \\
40000 \\
41300\end{array}$ & $\begin{array}{l}35000 \\
40400 \\
38500\end{array}$ & $\begin{array}{l}36300 \\
41000 \\
44100\end{array}$ & $\begin{array}{l}37500 \\
40000 \\
42700\end{array}$ & $\begin{array}{l}40744 \\
40744 \\
40744\end{array}$ \\
\hline CLALLAM & $\begin{array}{l}\text { VITAL } \\
\text { RATIO } \\
\text { COMPONENT II }\end{array}$ & $\begin{array}{l}29600 \\
27400 \\
31400\end{array}$ & $\begin{array}{l}29500 \\
28107 \\
31700\end{array}$ & $\begin{array}{l}29300 \\
28700 \\
31900\end{array}$ & $\begin{array}{l}30900 \\
28100 \\
27700\end{array}$ & $\begin{array}{l}30300 \\
28100 \\
30700\end{array}$ & $\begin{array}{l}31600 \\
28100 \\
30300\end{array}$ & $\begin{array}{l}30022 \\
30022 \\
30022\end{array}$ \\
\hline CLARK & $\begin{array}{l}\text { VITAL } \\
\text { RATIO } \\
\text { COMPONENT II }\end{array}$ & $\begin{array}{l}80800 \\
85400 \\
92400\end{array}$ & $\begin{array}{l}86500 \\
88550 \\
86900\end{array}$ & $\begin{array}{l}86300 \\
90000 \\
88400\end{array}$ & $\begin{array}{l}82500 \\
88700 \\
81000\end{array}$ & $\begin{array}{l}89000 \\
89700 \\
91100\end{array}$ & $\begin{array}{l}88000 \\
91400 \\
91700\end{array}$ & $\begin{array}{l}93809 \\
93809 \\
93809\end{array}$ \\
\hline COLUMBIA & $\begin{array}{l}\text { VITAL } \\
\text { RATIO } \\
\text { COMPONENT II }\end{array}$ & $\begin{array}{l}4200 \\
4300 \\
4800\end{array}$ & $\begin{array}{l}4400 \\
4258 \\
4800\end{array}$ & $\begin{array}{l}4300 \\
4300 \\
4600\end{array}$ & $\begin{array}{l}4700 \\
4400 \\
4300\end{array}$ & $\begin{array}{l}4800 \\
4400 \\
4800\end{array}$ & $\begin{array}{l}4100 \\
4200 \\
4900\end{array}$ & $\begin{array}{l}4569 \\
4569 \\
4569\end{array}$ \\
\hline COWLITZ & $\begin{array}{l}\text { VITAL } \\
\text { RATIO } \\
\text { COMPONENT II }\end{array}$ & $\begin{array}{l}54200 \\
56900 \\
57900\end{array}$ & $\begin{array}{l}54500 \\
57444 \\
59000\end{array}$ & $\begin{array}{l}57100 \\
58600 \\
59800\end{array}$ & $\begin{array}{l}55600 \\
57900 \\
54000\end{array}$ & $\begin{array}{l}54200 \\
57600 \\
58600\end{array}$ & $\begin{array}{l}58900 \\
57100 \\
57500\end{array}$ & $\begin{array}{l}57801 \\
57801 \\
57801\end{array}$ \\
\hline DOUGLAS & $\begin{array}{l}\text { VITAL } \\
\text { RATIO } \\
\text { COMPONENT II }\end{array}$ & $\begin{array}{l}14700 \\
12800 \\
12000\end{array}$ & $\begin{array}{l}11900 \\
12701 \\
13100\end{array}$ & $\begin{array}{l}12700 \\
12900 \\
12900\end{array}$ & $\begin{array}{l}12800 \\
13400 \\
11500\end{array}$ & $\begin{array}{l}14300 \\
14000 \\
14500\end{array}$ & $\begin{array}{l}14900 \\
13700 \\
14700\end{array}$ & $\begin{array}{l}14890 \\
14890 \\
14890\end{array}$ \\
\hline FERRY & $\begin{array}{l}\text { VITAL } \\
\text { RATIO } \\
\text { COMPONENT II }\end{array}$ & $\begin{array}{l}5000 \\
3900 \\
4100\end{array}$ & $\begin{array}{l}4500 \\
3583 \\
3600\end{array}$ & $\begin{array}{l}5100 \\
3600 \\
3600\end{array}$ & $\begin{array}{l}5100 \\
3700 \\
3400\end{array}$ & $\begin{array}{l}4900 \\
3800 \\
3700\end{array}$ & $\begin{array}{l}5000 \\
3700 \\
3900\end{array}$ & $\begin{array}{l}3889 \\
3889 \\
3889\end{array}$ \\
\hline FRANKL IN & $\begin{array}{l}\text { VITAL } \\
\text { RATIO } \\
\text { COMPONENT II }\end{array}$ & $\begin{array}{l}17100 \\
17000 \\
19000\end{array}$ & $\begin{array}{l}19300 \\
19202 \\
22000\end{array}$ & $\begin{array}{l}18400 \\
19700 \\
22200\end{array}$ & $\begin{array}{l}16900 \\
20800 \\
20300\end{array}$ & $\begin{array}{l}18500 \\
21300 \\
23600\end{array}$ & $\begin{array}{l}17500 \\
22000 \\
23800\end{array}$ & $\begin{array}{l}23342 \\
23342 \\
23342\end{array}$ \\
\hline GARF IELD & $\begin{array}{l}\text { VITAL } \\
\text { RATIO } \\
\text { COMPONENT II }\end{array}$ & $\begin{array}{l}3100 \\
2900 \\
3400\end{array}$ & $\begin{array}{l}2900 \\
2735 \\
3100\end{array}$ & $\begin{array}{l}2900 \\
2700 \\
3100\end{array}$ & $\begin{array}{l}3200 \\
2800 \\
2900\end{array}$ & $\begin{array}{l}3200 \\
2800 \\
3100\end{array}$ & $\begin{array}{l}2800 \\
2700 \\
3000\end{array}$ & $\begin{array}{l}2976 \\
2976 \\
2976\end{array}$ \\
\hline
\end{tabular}


TABLE 4c. (contd)

\begin{tabular}{|c|c|c|c|c|c|c|c|c|}
\hline & & 1954 & 1955 & 1956 & 1957 & 1958 & 1959 & $1960^{(b)}$ \\
\hline GRANT & $\begin{array}{l}\text { VITAL } \\
\text { RATIO } \\
\text { COMPONENT II }\end{array}$ & $\begin{array}{l}32800 \\
33200 \\
37200\end{array}$ & $\begin{array}{l}37700 \\
39964 \\
47000\end{array}$ & $\begin{array}{l}41000 \\
41600 \\
50100\end{array}$ & $\begin{array}{l}41200 \\
42200 \\
40100\end{array}$ & $\begin{array}{l}40000 \\
42400 \\
45300\end{array}$ & $\begin{array}{l}40600 \\
43600 \\
44600\end{array}$ & $\begin{array}{l}46477 \\
46477 \\
46477\end{array}$ \\
\hline $\begin{array}{l}\text { GRAYS } \\
\text { HARBOR }\end{array}$ & $\begin{array}{l}\text { VITAL } \\
\text { RATIO } \\
\text { COMPONENT II }\end{array}$ & $\begin{array}{l}47400 \\
51100 \\
55900\end{array}$ & $\begin{array}{l}48300 \\
51410 \\
55000\end{array}$ & $\begin{array}{l}51300 \\
53100 \\
55900\end{array}$ & $\begin{array}{l}50500 \\
52600 \\
49000\end{array}$ & $\begin{array}{l}50900 \\
53400 \\
54800\end{array}$ & $\begin{array}{l}51000 \\
52400 \\
54100\end{array}$ & $\begin{array}{l}54465 \\
54465 \\
54465\end{array}$ \\
\hline ISLAND & $\begin{array}{l}\text { VITAL } \\
\text { RATIO } \\
\text { COMPONENT II }\end{array}$ & $\begin{array}{l}17900 \\
14900 \\
15200\end{array}$ & $\begin{array}{l}19100 \\
14942 \\
12000\end{array}$ & $\begin{array}{l}18300 \\
15600 \\
13000\end{array}$ & $\begin{array}{l}20600 \\
16700 \\
12900\end{array}$ & $\begin{array}{l}21400 \\
17800 \\
16200\end{array}$ & $\begin{array}{l}23300 \\
18800 \\
17600\end{array}$ & $\begin{array}{l}19638 \\
19638 \\
19638\end{array}$ \\
\hline JEFFERSON & $\begin{array}{l}\text { VITAL } \\
\text { RATIO } \\
\text { COMPONENT II }\end{array}$ & $\begin{array}{r}9800 \\
11100 \\
10800\end{array}$ & $\begin{array}{l}10600 \\
10913 \\
10400\end{array}$ & $\begin{array}{r}9600 \\
11000 \\
10000\end{array}$ & $\begin{array}{r}10100 \\
11200 \\
9300\end{array}$ & $\begin{array}{r}9500 \\
11100 \\
9800\end{array}$ & $\begin{array}{r}8800 \\
11200 \\
9800\end{array}$ & $\begin{array}{l}9639 \\
9639 \\
9639\end{array}$ \\
\hline KING & $\begin{array}{l}\text { VITAL } \\
\text { RATIO } \\
\text { COMPONENT II }\end{array}$ & $\begin{array}{l}768200 \\
789300 \\
834900\end{array}$ & $\begin{array}{l}807200 \\
829585 \\
846700\end{array}$ & $\begin{array}{l}832500 \\
855800 \\
865200\end{array}$ & $\begin{array}{l}863600 \\
887000 \\
811700\end{array}$ & $\begin{array}{l}897000 \\
907200 \\
922400\end{array}$ & $\begin{array}{l}919500 \\
946400 \\
929500\end{array}$ & $\begin{array}{l}935014 \\
935014 \\
935014\end{array}$ \\
\hline KITSAP & $\begin{array}{l}\text { VITAL } \\
\text { RATIO } \\
\text { COMPONENT II }\end{array}$ & $\begin{array}{l}86900 \\
80200 \\
87600\end{array}$ & $\begin{array}{l}87300 \\
80757 \\
94800\end{array}$ & $\begin{array}{l}86100 \\
80800 \\
92400\end{array}$ & $\begin{array}{l}86500 \\
79300 \\
81300\end{array}$ & $\begin{array}{l}83700 \\
79800 \\
90500\end{array}$ & $\begin{array}{l}82700 \\
77200 \\
87000\end{array}$ & $\begin{array}{l}84176 \\
84176 \\
84176\end{array}$ \\
\hline KITYITAS & $\begin{array}{l}\text { VITAL } \\
\text { RATIO } \\
\text { COMPONENT II }\end{array}$ & $\begin{array}{l}18000 \\
20000 \\
21600\end{array}$ & $\begin{array}{l}20600 \\
19744 \\
19400\end{array}$ & $\begin{array}{l}20800 \\
19800 \\
19200\end{array}$ & $\begin{array}{l}20000 \\
19500 \\
16900\end{array}$ & $\begin{array}{l}22000 \\
20000 \\
18700\end{array}$ & $\begin{array}{l}20300 \\
19900 \\
20200\end{array}$ & $\begin{array}{l}20467 \\
20467 \\
20467\end{array}$ \\
\hline KLICKITAT & $\begin{array}{l}\text { VITAL } \\
\text { RATIO } \\
\text { COMPONENT II }\end{array}$ & $\begin{array}{l}13900 \\
13100 \\
17000\end{array}$ & $\begin{array}{l}13700 \\
13624 \\
16600\end{array}$ & $\begin{array}{l}11400 \\
13000 \\
14000\end{array}$ & $\begin{array}{l}11900 \\
12500 \\
12200\end{array}$ & $\begin{array}{l}11100 \\
12400 \\
13600\end{array}$ & $\begin{array}{l}11800 \\
12900 \\
13200\end{array}$ & $\begin{array}{l}13455 \\
13455 \\
13455\end{array}$ \\
\hline LEWIS & $\begin{array}{l}\text { VITAL } \\
\text { RATIO } \\
\text { COMPONENT II }\end{array}$ & $\begin{array}{l}42200 \\
41100 \\
46300\end{array}$ & $\begin{array}{l}41400 \\
41065 \\
43600\end{array}$ & $\begin{array}{l}43000 \\
41300 \\
43300\end{array}$ & $\begin{array}{l}42900 \\
41600 \\
38200\end{array}$ & $\begin{array}{l}40400 \\
41200 \\
40400\end{array}$ & $\begin{array}{l}42600 \\
40200 \\
41300\end{array}$ & $\begin{array}{l}41858 \\
41858 \\
41858\end{array}$ \\
\hline LINCOLN & $\begin{array}{l}\text { VITAL } \\
\text { RATIO } \\
\text { COMPONENT II }\end{array}$ & $\begin{array}{l}11000 \\
10500 \\
11100\end{array}$ & $\begin{array}{l}11900 \\
10408 \\
10100\end{array}$ & $\begin{array}{r}12200 \\
10500 \\
9900\end{array}$ & $\begin{array}{r}10200 \\
10700 \\
9500\end{array}$ & $\begin{array}{l}10600 \\
10700 \\
10800\end{array}$ & $\begin{array}{l}10600 \\
10800 \\
11300\end{array}$ & $\begin{array}{l}10919 \\
10919 \\
10919\end{array}$ \\
\hline MASON & $\begin{array}{l}\text { VITAL } \\
\text { RATIO } \\
\text { COMPONENT II }\end{array}$ & $\begin{array}{l}18600 \\
15400 \\
17000\end{array}$ & $\begin{array}{l}19300 \\
15625 \\
16600\end{array}$ & $\begin{array}{l}21100 \\
15800 \\
17100\end{array}$ & $\begin{array}{l}19500 \\
15900 \\
15100\end{array}$ & $\begin{array}{l}20700 \\
15800 \\
16800\end{array}$ & $\begin{array}{l}17100 \\
15400 \\
16700\end{array}$ & $\begin{array}{l}16251 \\
16251 \\
16251\end{array}$ \\
\hline OKANOC & $\begin{array}{l}\text { VITAL } \\
\text { RATIO } \\
\text { COMPONENT II }\end{array}$ & $\begin{array}{l}25500 \\
27000 \\
27700\end{array}$ & $\begin{array}{l}23300 \\
25920 \\
26900\end{array}$ & $\begin{array}{l}25500 \\
26200 \\
25600\end{array}$ & $\begin{array}{l}26300 \\
25800 \\
22800\end{array}$ & $\begin{array}{l}23200 \\
25900 \\
25600\end{array}$ & $\begin{array}{l}25100 \\
25400 \\
25400\end{array}$ & $\begin{array}{l}25520 \\
25520 \\
25520\end{array}$ \\
\hline
\end{tabular}


TABLE 4c. (contd)

\begin{tabular}{|c|c|c|c|c|c|c|c|c|}
\hline & & 1954 & 1955 & 1956 & 1957 & 1958 & 1959 & $1960^{(\mathrm{b})}$ \\
\hline CIFIC & $\begin{array}{l}\text { VITAL } \\
\text { RATIO } \\
\text { COMPONENT' II }\end{array}$ & $\begin{array}{l}15000 \\
15600 \\
17000\end{array}$ & $\begin{array}{l}16800 \\
15271 \\
15800\end{array}$ & $\begin{array}{l}15300 \\
15400 \\
15700\end{array}$ & $\begin{array}{l}16000 \\
15300 \\
14100\end{array}$ & $\begin{array}{l}15200 \\
15400 \\
15500\end{array}$ & $\begin{array}{l}16700 \\
14400 \\
14800\end{array}$ & $\begin{array}{l}14674 \\
14674 \\
14674\end{array}$ \\
\hline $\begin{array}{l}\text { END } \\
\text { ORIELLE }\end{array}$ & $\begin{array}{l}\text { VITAL } \\
\text { RATIO } \\
\text { COMPONENT II }\end{array}$ & $\begin{array}{l}8300 \\
7700 \\
9200\end{array}$ & $\begin{array}{l}8100 \\
7286 \\
7400\end{array}$ & $\begin{array}{l}8400 \\
7600 \\
8200\end{array}$ & $\begin{array}{l}9000 \\
7400 \\
7500\end{array}$ & $\begin{array}{l}7700 \\
7200 \\
7800\end{array}$ & $\begin{array}{l}7000 \\
7000 \\
7400\end{array}$ & $\begin{array}{l}6914 \\
6914 \\
6914\end{array}$ \\
\hline ERCE & $\begin{array}{l}\text { VITAL } \\
\text { RATIO } \\
\text { COMPONENT II }\end{array}$ & $\begin{array}{l}284700 \\
291700 \\
296500\end{array}$ & $\begin{array}{l}294800 \\
298538 \\
288700\end{array}$ & $\begin{array}{l}317600 \\
311400 \\
291900\end{array}$ & $\begin{array}{l}345600 \\
318700 \\
260700\end{array}$ & $\begin{array}{l}338900 \\
323700 \\
298500\end{array}$ & $\begin{array}{l}355300 \\
330800 \\
304000\end{array}$ & $\begin{array}{l}321590 \\
321590 \\
321590\end{array}$ \\
\hline N JUAN & $\begin{array}{l}\text { VITAL } \\
\text { RATIO } \\
\text { COMPONENT II }\end{array}$ & $\begin{array}{l}2700 \\
2900 \\
2900\end{array}$ & $\begin{array}{l}2600 \\
2823 \\
2700\end{array}$ & $\begin{array}{l}2900 \\
2800 \\
2800\end{array}$ & $\begin{array}{l}3000 \\
2800 \\
2500\end{array}$ & $\begin{array}{l}2200 \\
2900 \\
2800\end{array}$ & $\begin{array}{l}3200 \\
2800 \\
2800\end{array}$ & $\begin{array}{l}2872 \\
2872 \\
2872\end{array}$ \\
\hline AGI & $\begin{array}{l}\text { VITAL } \\
\text { RATIO } \\
\text { COMPONENT I I }\end{array}$ & $\begin{array}{l}43900 \\
43600 \\
51000\end{array}$ & $\begin{array}{l}47700 \\
46202 \\
50700\end{array}$ & $\begin{array}{l}48400 \\
46800 \\
49600\end{array}$ & $\begin{array}{l}47000 \\
48500 \\
47900\end{array}$ & $\begin{array}{l}49700 \\
49200 \\
53200\end{array}$ & $\begin{array}{l}47900 \\
49700 \\
52300\end{array}$ & $\begin{array}{l}51350 \\
51350 \\
51350\end{array}$ \\
\hline AMANIA & $\begin{array}{l}\text { VITAL } \\
\text { RATIO } \\
\text { COMPONENT II }\end{array}$ & $\begin{array}{l}4700 \\
4800 \\
4800\end{array}$ & $\begin{array}{l}5000 \\
4886 \\
5000\end{array}$ & $\begin{array}{l}5300 \\
5000 \\
4900\end{array}$ & $\begin{array}{l}5500 \\
4900 \\
4100\end{array}$ & $\begin{array}{l}4700 \\
5000 \\
5000\end{array}$ & $\begin{array}{l}6000 \\
5100 \\
5200\end{array}$ & $\begin{array}{l}5207 \\
5207 \\
5207\end{array}$ \\
\hline IOHOMISH & $\begin{array}{l}\text { VITAL } \\
\text { RATIO } \\
\text { COMPONENT II }\end{array}$ & $\begin{array}{l}129200 \\
125700 \\
133800\end{array}$ & $\begin{array}{l}134700 \\
136516 \\
136300\end{array}$ & $\begin{array}{l}144700 \\
141300 \\
141500\end{array}$ & $\begin{array}{l}149000 \\
147900 \\
133400\end{array}$ & $\begin{array}{l}155900 \\
152700 \\
159400\end{array}$ & $\begin{array}{l}160300 \\
161500 \\
165600\end{array}$ & $\begin{array}{l}172199 \\
172199 \\
172199\end{array}$ \\
\hline OKANE & $\begin{array}{l}\text { VITAL } \\
\text { RATIO } \\
\text { COMPONENT II }\end{array}$ & $\begin{array}{l}259300 \\
243700 \\
269400\end{array}$ & $\begin{array}{l}264900 \\
253361 \\
264700\end{array}$ & $\begin{array}{l}275800 \\
259900 \\
270900\end{array}$ & $\begin{array}{l}276800 \\
266800 \\
250700\end{array}$ & $\begin{array}{l}274900 \\
271500 \\
284100\end{array}$ & $\begin{array}{l}276500 \\
273400 \\
281100\end{array}$ & $\begin{array}{l}278333 \\
278333 \\
278333\end{array}$ \\
\hline EVENS & $\begin{array}{l}\text { VITAL } \\
\text { RATIO } \\
\text { COMPONENT II }\end{array}$ & $\begin{array}{l}19400 \\
17700 \\
18500\end{array}$ & $\begin{array}{l}19500 \\
17845 \\
18400\end{array}$ & $\begin{array}{l}20000 \\
18000 \\
18000\end{array}$ & $\begin{array}{l}18000 \\
18000 \\
16500\end{array}$ & $\begin{array}{l}18600 \\
18400 \\
19200\end{array}$ & $\begin{array}{l}19400 \\
17600 \\
18900\end{array}$ & $\begin{array}{l}17884 \\
17884 \\
17884\end{array}$ \\
\hline THURSTON & $\begin{array}{l}\text { VITAL } \\
\text { RATIO } \\
\text { COMPONENT II }\end{array}$ & $\begin{array}{l}47900 \\
45000 \\
47800\end{array}$ & $\begin{array}{l}47000 \\
45793 \\
48800\end{array}$ & $\begin{array}{l}52000 \\
48000 \\
49900\end{array}$ & $\begin{array}{l}53600 \\
49200 \\
45200\end{array}$ & $\begin{array}{l}55900 \\
51000 \\
53100\end{array}$ & $\begin{array}{l}56500 \\
50900 \\
53900\end{array}$ & $\begin{array}{l}55049 \\
55049 \\
55049\end{array}$ \\
\hline AHKIAKUM & $\begin{array}{l}\text { VITAL } \\
\text { RATIO } \\
\text { COMPONENT II }\end{array}$ & $\begin{array}{l}4000 \\
3700 \\
3600\end{array}$ & $\begin{array}{l}4600 \\
3656 \\
3400\end{array}$ & $\begin{array}{l}3300 \\
3500 \\
3100\end{array}$ & $\begin{array}{l}4300 \\
3700 \\
3000\end{array}$ & $\begin{array}{l}3900 \\
3700 \\
3400\end{array}$ & $\begin{array}{l}3500 \\
3500 \\
3500\end{array}$ & $\begin{array}{l}3426 \\
3426 \\
3426\end{array}$ \\
\hline $\begin{array}{l}\text { ALLA } \\
\text { WALLA }\end{array}$ & $\begin{array}{l}\text { VITAL } \\
\text { RATI0 } \\
\text { COMPONENT II }\end{array}$ & $\begin{array}{l}38300 \\
40600 \\
42100\end{array}$ & $\begin{array}{l}39500 \\
40867 \\
41200\end{array}$ & $\begin{array}{l}37000 \\
41200 \\
41500\end{array}$ & $\begin{array}{l}41200 \\
41600 \\
37500\end{array}$ & $\begin{array}{l}42200 \\
42100 \\
41300\end{array}$ & $\begin{array}{l}40100 \\
41600 \\
41900\end{array}$ & $\begin{array}{l}42195 \\
42195 \\
42195\end{array}$ \\
\hline
\end{tabular}


TABLE 4C. (contd)

\begin{tabular}{|c|c|c|c|c|c|c|c|c|}
\hline & & 1954. & 1955 & 1956 & 1957 & 1958 & 1959 & $1960^{(b)}$ \\
\hline WHATCOM & $\begin{array}{l}\text { VITAL } \\
\text { RATIO } \\
\text { COMPONENT II }\end{array}$ & $\begin{array}{l}64200 \\
66100 \\
71000\end{array}$ & $\begin{array}{l}67500 \\
66317 \\
70100\end{array}$ & $\begin{array}{l}66000 \\
66100 \\
68000\end{array}$ & $\begin{array}{l}62300 \\
67100 \\
63200\end{array}$ & $\begin{array}{l}67800 \\
68200 \\
71200\end{array}$ & $\begin{array}{l}66100 \\
66400 \\
70300\end{array}$ & $\begin{array}{l}70317 \\
70317 \\
70317\end{array}$ \\
\hline WHITMAN & $\begin{array}{l}\text { VITAL } \\
\text { RATIO } \\
\text { COMPONENT II }\end{array}$ & $\begin{array}{l}28500 \\
31100 \\
32700\end{array}$ & $\begin{array}{l}31300 \\
30902 \\
30800\end{array}$ & $\begin{array}{l}28900 \\
31200 \\
29900\end{array}$ & $\begin{array}{l}31700 \\
32000 \\
28700\end{array}$ & $\begin{array}{l}31200 \\
32600 \\
31000\end{array}$ & $\begin{array}{l}31000 \\
31800 \\
31200\end{array}$ & $\begin{array}{l}31263 \\
31263 \\
31263\end{array}$ \\
\hline YAKIMA & $\begin{array}{l}\text { VITAL } \\
\text { RATIO } \\
\text { COMPONENT II }\end{array}$ & $\begin{array}{l}136300 \\
134100 \\
157700\end{array}$ & $\begin{array}{l}145600 \\
140223 \\
158900\end{array}$ & $\begin{array}{l}136400 \\
140900 \\
154100\end{array}$ & $\begin{array}{l}138200 \\
140600 \\
134500\end{array}$ & $\begin{array}{l}145300 \\
143900 \\
151600\end{array}$ & $\begin{array}{l}146600 \\
142400 \\
147700\end{array}$ & $\begin{array}{l}145112 \\
145112 \\
145112\end{array}$ \\
\hline
\end{tabular}

(a) Intercensus estimates are rounded to nearest hundred.

(b) Actual census count.

(c) Average of birth and death estimates. 
TABLE 4d. Population Estimates for Washington Counties $1961-1967^{\text {(a) }}$

\begin{tabular}{|c|c|c|c|c|c|c|c|c|}
\hline & & 1961 & 1962 & 1963 & 1964 & 1965 & 1966 & 1967 \\
\hline ADAMS & $\begin{array}{l}\text { VITAL }{ }^{(b)} \\
\text { RATIO } \\
\text { COMPONENT II }\end{array}$ & $\begin{array}{r}8800 \\
10400 \\
9400\end{array}$ & $\begin{array}{r}8800 \\
10900 \\
9700\end{array}$ & $\begin{array}{r}8500 \\
10500 \\
9800\end{array}$ & $\begin{array}{r}9800 \\
10700 \\
9900\end{array}$ & $\begin{array}{r}8700 \\
10400 \\
10000\end{array}$ & $\begin{array}{r}9500 \\
10800 \\
10100\end{array}$ & $\begin{array}{l}10100 \\
10900 \\
10200\end{array}$ \\
\hline ASOTIN & $\begin{array}{l}\text { VITAL } \\
\text { RATIO } \\
\text { COMPONENT II }\end{array}$ & $\begin{array}{l}11300 \\
12300 \\
11500\end{array}$ & $\begin{array}{l}11200 \\
12400 \\
11500\end{array}$ & $\begin{array}{l}12500 \\
12500 \\
11400\end{array}$ & $\begin{array}{l}13500 \\
12600 \\
11100\end{array}$ & $\begin{array}{l}12600 \\
12700 \\
11100\end{array}$ & $\begin{array}{l}12000 \\
12900 \\
11300\end{array}$ & $\begin{array}{l}12500 \\
13200 \\
12100\end{array}$ \\
\hline BENTON & $\begin{array}{l}\text { VITAL } \\
\text { RATIO } \\
\text { COMPONENT II }\end{array}$ & $\begin{array}{l}62200 \\
62300 \\
56900\end{array}$ & $\begin{array}{l}64000 \\
62700 \\
57800\end{array}$ & $\begin{array}{l}67000 \\
64400 \\
55600\end{array}$ & $\begin{array}{l}65100 \\
64100 \\
53400\end{array}$ & $\begin{array}{l}63800 \\
64600 \\
52500\end{array}$ & $\begin{array}{l}68900 \\
65400 \\
52300\end{array}$ & $\begin{array}{l}66300 \\
65800 \\
54200\end{array}$ \\
\hline IELAN & $\begin{array}{l}\text { VITAL } \\
\text { RATI0 } \\
\text { COMPONENT II }\end{array}$ & $\begin{array}{l}42400 \\
40300 \\
35700\end{array}$ & $\begin{array}{l}40200 \\
39600 \\
35700\end{array}$ & $\begin{array}{l}43700 \\
40000 \\
34800\end{array}$ & $\begin{array}{l}46000 \\
40000 \\
34200\end{array}$ & $\begin{array}{l}41900 \\
39100 \\
31700\end{array}$ & $\begin{array}{l}43800 \\
39400 \\
31100\end{array}$ & $\begin{array}{l}47300 \\
40200 \\
31700\end{array}$ \\
\hline ALLAM & $\begin{array}{l}\text { VITAL } \\
\text { RATIO } \\
\text { COMPONENT II }\end{array}$ & $\begin{array}{l}29800 \\
30800 \\
27500\end{array}$ & $\begin{array}{l}29500 \\
30500 \\
28400\end{array}$ & $\begin{array}{l}26600 \\
30600 \\
28700\end{array}$ & $\begin{array}{l}28800 \\
30700 \\
29300\end{array}$ & $\begin{array}{l}32400 \\
31500 \\
29600\end{array}$ & $\begin{array}{l}29800 \\
31300 \\
28900\end{array}$ & $\begin{array}{l}31500 \\
32600 \\
29900\end{array}$ \\
\hline ARK & $\begin{array}{l}\text { VITAL } \\
\text { RATI0 } \\
\text { COMPONENT II }\end{array}$ & $\begin{array}{l}98500 \\
93600 \\
84200\end{array}$ & $\begin{array}{l}99400 \\
95300 \\
84800\end{array}$ & $\begin{array}{l}97300 \\
97500 \\
84000\end{array}$ & $\begin{array}{r}107000 \\
99000 \\
83800\end{array}$ & $\begin{array}{r}106100 \\
100100 \\
84500\end{array}$ & $\begin{array}{r}116400 \\
105600 \\
87700\end{array}$ & $\begin{array}{r}117600 \\
111200 \\
93000\end{array}$ \\
\hline COLUMBIA & $\begin{array}{l}\text { VITAL } \\
\text { RATIO } \\
\text { COMPONENT II }\end{array}$ & $\begin{array}{l}5600 \\
4800 \\
4200\end{array}$ & $\begin{array}{l}5800 \\
4800 \\
4100\end{array}$ & $\begin{array}{l}6100 \\
5000 \\
4300\end{array}$ & $\begin{array}{l}5300 \\
4900 \\
4000\end{array}$ & $\begin{array}{l}5300 \\
4700 \\
4300\end{array}$ & $\begin{array}{l}6000 \\
5100 \\
4900\end{array}$ & $\begin{array}{l}6100 \\
5600 \\
4900\end{array}$ \\
\hline COWLITZ & $\begin{array}{l}\text { VITAL } \\
\text { RATIO } \\
\text { COMPONENT II }\end{array}$ & $\begin{array}{l}56500 \\
56600 \\
52500\end{array}$ & $\begin{array}{l}57300 \\
57500 \\
52700\end{array}$ & $\begin{array}{l}59400 \\
57500 \\
52500\end{array}$ & $\begin{array}{l}61700 \\
59000 \\
52100\end{array}$ & $\begin{array}{l}67900 \\
61000 \\
53200\end{array}$ & $\begin{array}{l}62000 \\
62300 \\
54800\end{array}$ & $\begin{array}{l}64100 \\
63700 \\
56500\end{array}$ \\
\hline DOUGLAS & $\begin{array}{l}\text { VITAL } \\
\text { RATIO } \\
\text { COMPONENT II }\end{array}$ & $\begin{array}{l}14600 \\
15500 \\
13000\end{array}$ & $\begin{array}{l}14000 \\
15100 \\
12500\end{array}$ & $\begin{array}{l}15000 \\
14900 \\
12300\end{array}$ & $\begin{array}{l}15600 \\
14800 \\
12500\end{array}$ & $\begin{array}{l}17400 \\
15400 \\
12400\end{array}$ & $\begin{array}{l}19600 \\
15900 \\
12000\end{array}$ & $\begin{array}{l}18800 \\
15600 \\
12800\end{array}$ \\
\hline FERRY & $\begin{array}{l}\text { VITAL } \\
\text { RATIO } \\
\text { COMPONENT II }\end{array}$ & $\begin{array}{l}4100 \\
3800 \\
3300\end{array}$ & $\begin{array}{l}4200 \\
3800 \\
3400\end{array}$ & $\begin{array}{l}4200 \\
3700 \\
3400\end{array}$ & $\begin{array}{l}4100 \\
3700 \\
3400\end{array}$ & $\begin{array}{l}3900 \\
3600 \\
3300\end{array}$ & $\begin{array}{l}3600 \\
3500 \\
3200\end{array}$ & $\begin{array}{l}3400 \\
3400 \\
3200\end{array}$ \\
\hline FRANKLIN & $\begin{array}{l}\text { VITAL } \\
\text { RATIO } \\
\text { COMPONENT II }\end{array}$ & $\begin{array}{l}25000 \\
23800 \\
21500\end{array}$ & $\begin{array}{l}23100 \\
24300 \\
21600\end{array}$ & $\begin{array}{l}23300 \\
25700 \\
21000\end{array}$ & $\begin{array}{l}23800 \\
25100 \\
20700\end{array}$ & $\begin{array}{l}26000 \\
25100 \\
21000\end{array}$ & $\begin{array}{l}28100 \\
25800 \\
21200\end{array}$ & $\begin{array}{l}26800 \\
25200 \\
20700\end{array}$ \\
\hline RFIELD & $\begin{array}{l}\text { VITAL } \\
\text { RATIO } \\
\text { COMPONENT II }\end{array}$ & $\begin{array}{l}3400 \\
3300 \\
2600\end{array}$ & $\begin{array}{l}3000 \\
3000 \\
2600\end{array}$ & $\begin{array}{l}2200 \\
2800 \\
2600\end{array}$ & $\begin{array}{l}2800 \\
2900 \\
2400\end{array}$ & $\begin{array}{l}2100 \\
2700 \\
2600\end{array}$ & $\begin{array}{l}2800 \\
3100 \\
3200\end{array}$ & $\begin{array}{l}3300 \\
3400 \\
3500\end{array}$ \\
\hline
\end{tabular}


TABLE 4d. (contd)

\begin{tabular}{|c|c|c|c|c|c|c|c|c|}
\hline & & 1961 & 1962 & 1963 & 1964 & 1965 & 1966 & 1967 \\
\hline GRANT & $\begin{array}{l}\text { VITAL } \\
\text { RATIO } \\
\text { COMPONENT II }\end{array}$ & $\begin{array}{l}51500 \\
47900 \\
45600\end{array}$ & $\begin{array}{l}52500 \\
48500 \\
41100\end{array}$ & $\begin{array}{l}48200 \\
46800 \\
40000\end{array}$ & $\begin{array}{l}48500 \\
45500 \\
39100\end{array}$ & $\begin{array}{l}44500 \\
41900 \\
37600\end{array}$ & $\begin{array}{l}38900 \\
39600 \\
33600\end{array}$ & $\begin{array}{l}41700 \\
40300 \\
35200\end{array}$ \\
\hline $\begin{array}{l}\text { GRAYS } \\
\text { HARBOR }\end{array}$ & $\begin{array}{l}\text { VITAL } \\
\text { RATIO } \\
\text { COMPONENT II }\end{array}$ & $\begin{array}{l}54600 \\
52900 \\
48600\end{array}$ & $\begin{array}{l}53400 \\
54600 \\
48100\end{array}$ & $\begin{array}{l}55300 \\
53300 \\
49300\end{array}$ & $\begin{array}{l}58200 \\
55000 \\
49600\end{array}$ & $\begin{array}{l}62000 \\
55700 \\
48700\end{array}$ & $\begin{array}{l}59900 \\
56800 \\
48300\end{array}$ & $\begin{array}{l}58800 \\
55800 \\
49200\end{array}$ \\
\hline ISLAND & $\begin{array}{l}\text { VITAL } \\
\text { RATIO } \\
\text { COMPONENT II }\end{array}$ & $\begin{array}{l}20000 \\
20700 \\
19200\end{array}$ & $\begin{array}{l}19800 \\
21400 \\
19600\end{array}$ & $\begin{array}{l}20100 \\
22300 \\
20700\end{array}$ & $\begin{array}{l}21500 \\
22200 \\
19800\end{array}$ & $\begin{array}{l}19900 \\
21900 \\
20000\end{array}$ & $\begin{array}{l}20000 \\
22900 \\
21400\end{array}$ & $\begin{array}{l}23400 \\
25900 \\
22700\end{array}$ \\
\hline EFFERSON & $\begin{array}{l}\text { VITAL } \\
\text { RATIO } \\
\text { COMPONENT II }\end{array}$ & $\begin{array}{l}9300 \\
9600 \\
8300\end{array}$ & $\begin{array}{l}9300 \\
9800 \\
8600\end{array}$ & $\begin{array}{l}8100 \\
8900 \\
8800\end{array}$ & $\begin{array}{l}8500 \\
9500 \\
8300\end{array}$ & $\begin{array}{l}9200 \\
9400 \\
8000\end{array}$ & $\begin{array}{l}8800 \\
9700 \\
8200\end{array}$ & $\begin{array}{l}9500 \\
9900 \\
8200\end{array}$ \\
\hline ING & $\begin{array}{l}\text { VITAL } \\
\text { RATIO } \\
\text { COMPONENT II }\end{array}$ & $\begin{array}{l}942500 \\
953600 \\
868600\end{array}$ & $\begin{array}{l}968700 \\
979200 \\
885300\end{array}$ & $\begin{array}{l}970900 \\
993500 \\
882100\end{array}$ & $\begin{array}{l}947600 \\
985200 \\
878400\end{array}$ & $\begin{array}{l}952800 \\
986800 \\
898500\end{array}$ & $\begin{array}{r}1030100 \\
1033900 \\
926000\end{array}$ & $\begin{array}{r}1072900 \\
1093500 \\
983500\end{array}$ \\
\hline ITSAP & $\begin{array}{l}\text { VITAL } \\
\text { RATIO } \\
\text { COMPONENT I I }\end{array}$ & $\begin{array}{l}87200 \\
83600 \\
77000\end{array}$ & $\begin{array}{l}87000 \\
85600 \\
80600\end{array}$ & $\begin{array}{l}90300 \\
84900 \\
78700\end{array}$ & $\begin{array}{l}89000 \\
86400 \\
79300\end{array}$ & $\begin{array}{l}93600 \\
87800 \\
78300\end{array}$ & $\begin{array}{l}94400 \\
89900 \\
83000\end{array}$ & $\begin{array}{r}102100 \\
93700 \\
85400\end{array}$ \\
\hline KITTITAS & $\begin{array}{l}\text { VITAL } \\
\text { RATIO } \\
\text { COMPONENT II }\end{array}$ & $\begin{array}{l}20800 \\
21200 \\
19500\end{array}$ & $\begin{array}{l}19100 \\
20600 \\
19000\end{array}$ & $\begin{array}{l}19000 \\
19900 \\
19000\end{array}$ & $\begin{array}{l}18800 \\
19200 \\
19100\end{array}$ & $\begin{array}{l}19500 \\
19900 \\
19100\end{array}$ & $\begin{array}{l}19600 \\
20800 \\
19900\end{array}$ & $\begin{array}{l}19800 \\
20600 \\
20600\end{array}$ \\
\hline KLICKITAT & $\begin{array}{l}\text { VITAL } \\
\text { RATIO } \\
\text { COMPONENT I I }\end{array}$ & $\begin{array}{l}12600 \\
13400 \\
12300\end{array}$ & $\begin{array}{l}13100 \\
13500 \\
12000\end{array}$ & $\begin{array}{l}14000 \\
13600 \\
12000\end{array}$ & $\begin{array}{l}13200 \\
13500 \\
12200\end{array}$ & $\begin{array}{l}12000 \\
13700 \\
11500\end{array}$ & $\begin{array}{l}14000 \\
13800 \\
10500\end{array}$ & $\begin{array}{l}11500 \\
12200 \\
10000\end{array}$ \\
\hline LEWIS & $\begin{array}{l}\text { VITAL } \\
\text { RATIO } \\
\text { COMPONENT II }\end{array}$ & $\begin{array}{l}41200 \\
42500 \\
37200\end{array}$ & $\begin{array}{l}44600 \\
44100 \\
37500\end{array}$ & $\begin{array}{l}42300 \\
41900 \\
36500\end{array}$ & $\begin{array}{l}45400 \\
42200 \\
35500\end{array}$ & $\begin{array}{l}45600 \\
43500 \\
36200\end{array}$ & $\begin{array}{l}44300 \\
45500 \\
36300\end{array}$ & $\begin{array}{l}48400 \\
44200 \\
36300\end{array}$ \\
\hline LINCOLN & $\begin{array}{l}\text { VITAL } \\
\text { RATIO } \\
\text { COMPONENT II }\end{array}$ & $\begin{array}{r}10000 \\
10900 \\
9700\end{array}$ & $\begin{array}{r}9700 \\
10500 \\
9500\end{array}$ & $\begin{array}{r}10700 \\
10300 \\
8900\end{array}$ & $\begin{array}{l}9200 \\
9800 \\
8500\end{array}$ & $\begin{array}{l}9000 \\
9700 \\
8300\end{array}$ & $\begin{array}{r}10200 \\
9800 \\
8300\end{array}$ & $\begin{array}{l}9600 \\
9600 \\
8200\end{array}$ \\
\hline MASON & $\begin{array}{l}\text { VITAL } \\
\text { RATIO } \\
\text { COMPONENT II }\end{array}$ & $\begin{array}{l}17100 \\
16500 \\
14600\end{array}$ & $\begin{array}{l}17300 \\
16400 \\
14800\end{array}$ & $\begin{array}{l}17600 \\
16500 \\
15000\end{array}$ & $\begin{array}{l}17800 \\
17000 \\
15200\end{array}$ & $\begin{array}{l}18200 \\
17800 \\
15200\end{array}$ & $\begin{array}{l}17800 \\
17400 \\
15200\end{array}$ & $\begin{array}{l}18400 \\
18100 \\
15800\end{array}$ \\
\hline KANOGAN & $\begin{array}{l}\text { VITAL } \\
\text { RATIO } \\
\text { COMPONENT II }\end{array}$ & $\begin{array}{l}24800 \\
25400 \\
22100\end{array}$ & $\begin{array}{l}23000 \\
25000 \\
21900\end{array}$ & $\begin{array}{l}25500 \\
25000 \\
22100\end{array}$ & $\begin{array}{l}23800 \\
24700 \\
23000\end{array}$ & $\begin{array}{l}25400 \\
25200 \\
21500\end{array}$ & $\begin{array}{l}22700 \\
25500 \\
20800\end{array}$ & $\begin{array}{l}23400 \\
24300 \\
21200\end{array}$ \\
\hline
\end{tabular}


TABLE 4d. (contd)

\begin{tabular}{|c|c|c|c|c|c|c|c|c|}
\hline & & 1961 & 1962 & 1963 & 1964 & 1965 & 1966 & 1967 \\
\hline PACIFIC & $\begin{array}{l}\text { VITAL } \\
\text { RATIO } \\
\text { COMPONENT II }\end{array}$ & $\begin{array}{l}13500 \\
13900 \\
12900\end{array}$ & $\begin{array}{l}15500 \\
14900 \\
12700\end{array}$ & $\begin{array}{l}13400 \\
13400 \\
12100\end{array}$ & $\begin{array}{l}15700 \\
14200 \\
11900\end{array}$ & $\begin{array}{l}17700 \\
14700 \\
12000\end{array}$ & $\begin{array}{l}17800 \\
14600 \\
11500\end{array}$ & $\begin{array}{l}18800 \\
14800 \\
11900\end{array}$ \\
\hline $\begin{array}{l}\text { PEND } \\
\text { ORIELLE }\end{array}$ & $\begin{array}{l}\text { VITAL } \\
\text { RATIO } \\
\text { COMPONENT II }\end{array}$ & $\begin{array}{l}6900 \\
6900 \\
6400\end{array}$ & $\begin{array}{l}6500 \\
6700 \\
6400\end{array}$ & $\begin{array}{l}7100 \\
7100 \\
6800\end{array}$ & $\begin{array}{l}6900 \\
7300 \\
7200\end{array}$ & $\begin{array}{l}7900 \\
7800 \\
7600\end{array}$ & $\begin{array}{l}8200 \\
7900 \\
7300\end{array}$ & $\begin{array}{l}7800 \\
6700 \\
5300\end{array}$ \\
\hline PIERCE & $\begin{array}{l}\text { VITAL } \\
\text { RATIO } \\
\text { COMPONENT II }\end{array}$ & $\begin{array}{l}320600 \\
329200 \\
302000\end{array}$ & $\begin{array}{l}334600 \\
338500 \\
304100\end{array}$ & $\begin{array}{l}339200 \\
340700 \\
313000\end{array}$ & $\begin{array}{l}344100 \\
345300 \\
312900\end{array}$ & $\begin{array}{l}333000 \\
345200 \\
310500\end{array}$ & $\begin{array}{l}341300 \\
353800 \\
327600\end{array}$ & $\begin{array}{l}359800 \\
377100 \\
337600\end{array}$ \\
\hline SAN JUAN & $\begin{array}{l}\text { VITAL } \\
\text { RATIO } \\
\text { COMPONENT II }\end{array}$ & $\begin{array}{l}3000 \\
3100 \\
2400\end{array}$ & $\begin{array}{l}2900 \\
3000 \\
2400\end{array}$ & $\begin{array}{l}3200 \\
3100 \\
2200\end{array}$ & $\begin{array}{l}3800 \\
3100 \\
2000\end{array}$ & $\begin{array}{l}3600 \\
3300 \\
1800\end{array}$ & $\begin{array}{l}3300 \\
3300 \\
1800\end{array}$ & $\begin{array}{l}2700 \\
3100 \\
2100\end{array}$ \\
\hline SKAGIT & $\begin{array}{l}\text { VITAL } \\
\text { RATIO } \\
\text { COMPONENT II }\end{array}$ & $\begin{array}{l}52700 \\
50600 \\
45800\end{array}$ & $\begin{array}{l}53600 \\
50900 \\
45500\end{array}$ & $\begin{array}{l}50400 \\
49600 \\
45100\end{array}$ & $\begin{array}{l}51100 \\
49600 \\
43500\end{array}$ & $\begin{array}{l}50100 \\
49100 \\
42500\end{array}$ & $\begin{array}{l}52100 \\
51000 \\
42000\end{array}$ & $\begin{array}{l}55700 \\
51100 \\
43300\end{array}$ \\
\hline SKAMANIA & $\begin{array}{l}\text { VITAL } \\
\text { RATIO } \\
\text { COMPONENT II }\end{array}$ & $\begin{array}{l}4500 \\
5100 \\
4600\end{array}$ & $\begin{array}{l}4800 \\
5400 \\
4700\end{array}$ & $\begin{array}{l}5000 \\
5300 \\
4900\end{array}$ & $\begin{array}{l}5200 \\
5500 \\
4900\end{array}$ & $\begin{array}{l}5200 \\
5500 \\
4900\end{array}$ & $\begin{array}{l}4700 \\
5500 \\
4400\end{array}$ & $\begin{array}{l}5100 \\
5200 \\
4400\end{array}$ \\
\hline SNOHOMISH & $\begin{array}{l}\text { VITAL } \\
\text { RATIO } \\
\text { COMPONENT II }\end{array}$ & $\begin{array}{l}174300 \\
180400 \\
161200\end{array}$ & $\begin{array}{l}188000 \\
187400 \\
166700\end{array}$ & $\begin{array}{l}190100 \\
196200 \\
166400\end{array}$ & $\begin{array}{l}193300 \\
199100 \\
165800\end{array}$ & $\begin{array}{l}193500 \\
202900 \\
167700\end{array}$ & $\begin{array}{l}201300 \\
209700 \\
177700\end{array}$ & $\begin{array}{l}216900 \\
224000 \\
190900\end{array}$ \\
\hline SPOKANE & $\begin{array}{l}\text { VITAL } \\
\text { RATIO } \\
\text { COMPONENT II }\end{array}$ & $\begin{array}{l}276200 \\
272600 \\
248200\end{array}$ & $\begin{array}{l}274500 \\
273900 \\
246300\end{array}$ & $\begin{array}{l}274600 \\
268800 \\
239500\end{array}$ & $\begin{array}{l}273800 \\
267400 \\
236800\end{array}$ & $\begin{array}{l}275200 \\
264800 \\
234200\end{array}$ & $\begin{array}{l}269900 \\
269800 \\
235500\end{array}$ & $\begin{array}{l}272300 \\
272200 \\
242100\end{array}$ \\
\hline STEVENS & $\begin{array}{l}\text { VITAL } \\
\text { RATIO } \\
\text { COMPONENT II }\end{array}$ & $\begin{array}{l}18000 \\
17200 \\
15600\end{array}$ & $\begin{array}{l}16200 \\
17300 \\
15400\end{array}$ & $\begin{array}{l}15400 \\
15700 \\
15300\end{array}$ & $\begin{array}{l}16000 \\
16200 \\
14500\end{array}$ & $\begin{array}{l}17000 \\
16900 \\
14200\end{array}$ & $\begin{array}{l}16900 \\
17000 \\
13600\end{array}$ & $\begin{array}{l}16700 \\
16200 \\
13400\end{array}$ \\
\hline THURSTON & $\begin{array}{l}\text { VITAL } \\
\text { RATIO } \\
\text { COMPONENT II }\end{array}$ & $\begin{array}{l}58100 \\
56100 \\
53000\end{array}$ & $\begin{array}{l}64200 \\
59000 \\
52800\end{array}$ & $\begin{array}{l}61300 \\
58700 \\
52600\end{array}$ & $\begin{array}{l}65700 \\
60500 \\
53600\end{array}$ & $\begin{array}{l}62400 \\
61000 \\
54000\end{array}$ & $\begin{array}{l}67100 \\
62100 \\
57000\end{array}$ & $\begin{array}{l}70900 \\
66500 \\
60400\end{array}$ \\
\hline WAHKIAKUM & $\begin{array}{l}\text { VITAL } \\
\text { RATIO } \\
\text { COMPONENT II }\end{array}$ & $\begin{array}{l}3700 \\
3700 \\
3000\end{array}$ & $\begin{array}{l}3800 \\
3500 \\
3100\end{array}$ & $\begin{array}{l}4200 \\
3300 \\
2800\end{array}$ & $\begin{array}{l}3600 \\
3500 \\
3100\end{array}$ & $\begin{array}{l}4000 \\
3700 \\
2600\end{array}$ & $\begin{array}{l}3700 \\
3300 \\
2700\end{array}$ & $\begin{array}{l}4700 \\
3600 \\
2800\end{array}$ \\
\hline $\begin{array}{l}\text { WALLA } \\
\text { WALLA }\end{array}$ & $\begin{array}{l}\text { VITAL } \\
\text { RATIO } \\
\text { COMPONENT II }\end{array}$ & $\begin{array}{l}43600 \\
41700 \\
37600\end{array}$ & $\begin{array}{l}43800 \\
42700 \\
37600\end{array}$ & $\begin{array}{l}42400 \\
42000 \\
38700\end{array}$ & $\begin{array}{l}44400 \\
43300 \\
37100\end{array}$ & $\begin{array}{l}44400 \\
41300 \\
37500\end{array}$ & $\begin{array}{l}44300 \\
42900 \\
38000\end{array}$ & $\begin{array}{l}45400 \\
42500 \\
39000\end{array}$ \\
\hline
\end{tabular}


TABLE 4d. (contd)

\begin{tabular}{|c|c|c|c|c|c|c|c|c|}
\hline & & 1961 & 1962 & 1963 & 1964 & 1965 & 1966 & 1967 \\
\hline WHATCOM & $\begin{array}{l}\text { VITAL } \\
\text { RATIO } \\
\text { COMPONENT II }\end{array}$ & $\begin{array}{l}74500 \\
70600 \\
62700\end{array}$ & $\begin{array}{l}72600 \\
72200 \\
64700\end{array}$ & $\begin{array}{l}70900 \\
70900 \\
63700\end{array}$ & $\begin{array}{l}74300 \\
70700 \\
63300\end{array}$ & $\begin{array}{l}74200 \\
70900 \\
64400\end{array}$ & $\begin{array}{l}73600 \\
71000 \\
64400\end{array}$ & $\begin{array}{l}74800 \\
74500 \\
68900\end{array}$ \\
\hline WHITMAN & $\begin{array}{l}\text { VITAL } \\
\text { RATIO } \\
\text { COMPONENT II }\end{array}$ & $\begin{array}{l}30100 \\
31400 \\
29500\end{array}$ & $\begin{array}{l}31300 \\
32100 \\
30300\end{array}$ & $\begin{array}{l}31000 \\
32100 \\
31200\end{array}$ & & & & $\begin{array}{l}28600 \\
33300 \\
32200\end{array}$ \\
\hline YAKIMA & $\begin{array}{l}\text { VITAL } \\
\text { RATIO } \\
\text { COMPONENT II }\end{array}$ & $\begin{array}{l}149200 \\
143900 \\
133900\end{array}$ & $\begin{array}{l}151800 \\
145300 \\
133800\end{array}$ & $\begin{array}{l}158300 \\
146100 \\
132500\end{array}$ & & & & $\begin{array}{l}154700 \\
144400 \\
133600\end{array}$ \\
\hline
\end{tabular}

\footnotetext{
(a) Intercensus estimates are rounded to nearest hundred.

(b) Average of birth and death estimates.
} 
TABLE 4e. Population Estimates for Washington Counties $1968-1970^{(a)}$

\begin{tabular}{|c|c|c|c|c|}
\hline & & 1968 & 1969 & $1970^{(b)}$ \\
\hline ADAMS & $\begin{array}{l}\text { VITAL }{ }^{(c)} \\
\text { RATIO } \\
\text { COMPONENT II }\end{array}$ & $\begin{array}{r}9200 \\
11100 \\
10800\end{array}$ & $\begin{array}{r}9300 \\
10900 \\
12100\end{array}$ & $\begin{array}{l}12014 \\
12014 \\
12014\end{array}$ \\
\hline ASOTIN & $\begin{array}{l}\text { VITAL } \\
\text { RATIO } \\
\text { COMPONENT II }\end{array}$ & $\begin{array}{l}13200 \\
13100 \\
12600\end{array}$ & $\begin{array}{l}13100 \\
13200 \\
13500\end{array}$ & $\begin{array}{l}13799 \\
13799 \\
13799\end{array}$ \\
\hline BENTON & $\begin{array}{l}\text { VITAL } \\
\text { RATIO } \\
\text { COMPONENT II }\end{array}$ & $\begin{array}{l}65400 \\
66600 \\
61200\end{array}$ & $\begin{array}{l}71200 \\
66600 \\
67700\end{array}$ & $\begin{array}{l}67540 \\
67540 \\
67540\end{array}$ \\
\hline CHELAN & $\begin{array}{l}\text { VITAL } \\
\text { RATIO } \\
\text { COMPONENT II }\end{array}$ & $\begin{array}{l}42300 \\
39800 \\
38400\end{array}$ & $\begin{array}{l}40600 \\
39200 \\
40200\end{array}$ & $\begin{array}{l}41355 \\
41355 \\
41355\end{array}$ \\
\hline CLALLAM & $\begin{array}{l}\text { VITAL } \\
\text { RATIO } \\
\text { COMPONENT II }\end{array}$ & $\begin{array}{l}32300 \\
33300 \\
31800\end{array}$ & $\begin{array}{l}33100 \\
33800 \\
34600\end{array}$ & $\begin{array}{l}34770 \\
34770 \\
34770\end{array}$ \\
\hline CLARK & $\begin{array}{l}\text { VITAL } \\
\text { RATIO } \\
\text { COMPONENT II }\end{array}$ & $\begin{array}{l}121900 \\
116500 \\
109500\end{array}$ & $\begin{array}{l}125900 \\
121300 \\
124200\end{array}$ & $\begin{array}{l}128454 \\
128454 \\
128454\end{array}$ \\
\hline COLUMBIA & $\begin{array}{l}\text { VITAL } \\
\text { RATIO } \\
\text { COMPONENT II }\end{array}$ & $\begin{array}{l}4700 \\
4900 \\
5000\end{array}$ & $\begin{array}{l}5500 \\
4800 \\
4600\end{array}$ & $\begin{array}{l}4439 \\
4439 \\
4439\end{array}$ \\
\hline COWLITZ & $\begin{array}{l}\text { VITAL } \\
\text { RATIO } \\
\text { COMPONENT II }\end{array}$ & $\begin{array}{l}66200 \\
65500 \\
62900\end{array}$ & $\begin{array}{l}73300 \\
65900 \\
68100\end{array}$ & $\begin{array}{l}68616 \\
68616 \\
68616\end{array}$ \\
\hline DOUGLAS & $\begin{array}{l}\text { VITAL } \\
\text { RATIO } \\
\text { COMPONENT II }\end{array}$ & $\begin{array}{l}16800 \\
16800 \\
13800\end{array}$ & $\begin{array}{l}18600 \\
16600 \\
15200\end{array}$ & $\begin{array}{l}16787 \\
16787 \\
16787\end{array}$ \\
\hline FERRY & $\begin{array}{l}\text { VITAL } \\
\text { RATIO } \\
\text { COMPONENT II }\end{array}$ & $\begin{array}{l}3600 \\
3600 \\
3300\end{array}$ & $\begin{array}{l}3500 \\
3500 \\
3700\end{array}$ & $\begin{array}{l}3655 \\
3655 \\
3655\end{array}$ \\
\hline FRANKL IN & $\begin{array}{l}\text { VITAL } \\
\text { RATIO } \\
\text { COMPONENT II }\end{array}$ & $\begin{array}{l}25200 \\
25000 \\
24200\end{array}$ & $\begin{array}{l}27200 \\
26200 \\
25700\end{array}$ & $\begin{array}{l}25816 \\
25816 \\
25816\end{array}$ \\
\hline GARF IELD & $\begin{array}{l}\text { VITAL } \\
\text { RATIO } \\
\text { COMPONENT II }\end{array}$ & $\begin{array}{l}3100 \\
3400 \\
2800\end{array}$ & $\begin{array}{l}3100 \\
3100 \\
3000\end{array}$ & $\begin{array}{l}2911 \\
2911 \\
2911\end{array}$ \\
\hline
\end{tabular}


TABLE 4e. (contd)

\begin{tabular}{|c|c|c|c|c|}
\hline & & 1968 & 1969 & $1970^{(b)}$ \\
\hline GRANT & $\begin{array}{l}\text { VITAL } \\
\text { RATIO } \\
\text { COMPONENT II }\end{array}$ & $\begin{array}{l}42200 \\
41000 \\
39700\end{array}$ & $\begin{array}{l}43400 \\
41500 \\
42200\end{array}$ & $\begin{array}{l}41881 \\
41881 \\
41881\end{array}$ \\
\hline GRAYS HARBOR & $\begin{array}{l}\text { VITAL } \\
\text { RATIO } \\
\text { COMPONENT II }\end{array}$ & $\begin{array}{l}61200 \\
57100 \\
55100\end{array}$ & $\begin{array}{l}62100 \\
58700 \\
59600\end{array}$ & $\begin{array}{l}59553 \\
59553 \\
59553\end{array}$ \\
\hline ISLAND & $\begin{array}{l}\text { VITAL } \\
\text { RATIO } \\
\text { COMPONENT II }\end{array}$ & $\begin{array}{l}24100 \\
26500 \\
25300\end{array}$ & $\begin{array}{l}22700 \\
28400 \\
27300\end{array}$ & $\begin{array}{l}27011 \\
27011 \\
27011\end{array}$ \\
\hline JEFFERSON & $\begin{array}{l}\text { VITAL } \\
\text { RATIO } \\
\text { COMPONENT II }\end{array}$ & $\begin{array}{r}9000 \\
9900 \\
10200\end{array}$ & $\begin{array}{l}10400 \\
10600 \\
10800\end{array}$ & $\begin{array}{l}10661 \\
10661 \\
10661\end{array}$ \\
\hline KING & $\begin{array}{l}\text { VITAL } \\
\text { RATIO } \\
\text { COMPONENT II }\end{array}$ & $\begin{array}{l}1116500 \\
1129000 \\
1106800\end{array}$ & $\begin{array}{l}1112900 \\
1140400 \\
1193900\end{array}$ & $\begin{array}{l}1156633 \\
1156633 \\
1156633\end{array}$ \\
\hline KITSAP & $\begin{array}{l}\text { VITAL } \\
\text { RATIO } \\
\text { COMPONENT II }\end{array}$ & $\begin{array}{r}106700 \\
97300 \\
92600\end{array}$ & $\begin{array}{l}109300 \\
100000 \\
101500\end{array}$ & $\begin{array}{l}101732 \\
101732 \\
101732\end{array}$ \\
\hline KITTITAS & $\begin{array}{l}\text { VITAL } \\
\text { RATIO } \\
\text { COMPONENT II }\end{array}$ & $\begin{array}{l}20300 \\
21800 \\
23400\end{array}$ & $\begin{array}{l}20500 \\
22100 \\
24900\end{array}$ & $\begin{array}{l}25039 \\
25039 \\
25039\end{array}$ \\
\hline KLICKITAT & $\begin{array}{l}\text { VITAL } \\
\text { RATIO } \\
\text { COMPONENT I1 }\end{array}$ & $\begin{array}{l}11600 \\
12400 \\
11100\end{array}$ & $\begin{array}{l}12800 \\
12100 \\
12100\end{array}$ & $\begin{array}{l}12138 \\
12138 \\
12138\end{array}$ \\
\hline LEWIS & $\begin{array}{l}\text { VITAL } \\
\text { RATIO } \\
\text { COMPONENT II }\end{array}$ & $\begin{array}{l}46800 \\
45500 \\
41200\end{array}$ & $\begin{array}{l}48700 \\
46600 \\
45600\end{array}$ & $\begin{array}{l}45467 \\
45467 \\
45467\end{array}$ \\
\hline LINCOLN & $\begin{array}{l}\text { VITAL } \\
\text { RATIO } \\
\text { COMPONENT I I }\end{array}$ & $\begin{array}{r}9600 \\
10000 \\
9000\end{array}$ & $\begin{array}{r}10500 \\
9600 \\
9400\end{array}$ & $\begin{array}{l}9572 \\
9572 \\
9572\end{array}$ \\
\hline MASON & $\begin{array}{l}\text { VITAL } \\
\text { RAT10 } \\
\text { COMPONENT II }\end{array}$ & $\begin{array}{l}19500 \\
18800 \\
18700\end{array}$ & $\begin{array}{l}22100 \\
20000 \\
20300\end{array}$ & $\begin{array}{l}20918 \\
20918 \\
20918\end{array}$ \\
\hline OKANOGAN & $\begin{array}{l}\text { VITAL } \\
\text { RATIO } \\
\text { COMPONENT II }\end{array}$ & $\begin{array}{l}24300 \\
24400 \\
23600\end{array}$ & $\begin{array}{l}25800 \\
25300 \\
26100\end{array}$ & $\begin{array}{l}25867 \\
25867 \\
25867\end{array}$ \\
\hline
\end{tabular}


IABLE 4e. (contd)

\begin{tabular}{|c|c|c|c|c|}
\hline & & 1968 & 1969 & $1970^{(b)}$ \\
\hline PACIFIC & $\begin{array}{l}\text { VITAL } \\
\text { RATIO } \\
\text { COMPONENT II }\end{array}$ & $\begin{array}{l}17900 \\
14800 \\
14100\end{array}$ & $\begin{array}{l}18200 \\
15400 \\
15900\end{array}$ & $\begin{array}{l}15796 \\
15796 \\
15796\end{array}$ \\
\hline PEND OREILLE & $\begin{array}{l}\text { VITAL } \\
\text { RATIO } \\
\text { COMPONENT II }\end{array}$ & $\begin{array}{l}6000 \\
6400 \\
5300\end{array}$ & $\begin{array}{l}5700 \\
6200 \\
5800\end{array}$ & $\begin{array}{l}6025 \\
6025 \\
6025\end{array}$ \\
\hline PIERCE & $\begin{array}{l}\text { VITAL } \\
\text { RATIO } \\
\text { COMPONENT I I }\end{array}$ & $\begin{array}{l}372000 \\
388600 \\
389400\end{array}$ & $\begin{array}{l}386900 \\
402500 \\
418400\end{array}$ & $\begin{array}{l}411027 \\
411027 \\
411027\end{array}$ \\
\hline SAN JUAN & $\begin{array}{l}\text { VITAL } \\
\text { RATIO } \\
\text { COMPONENT II }\end{array}$ & $\begin{array}{l}2800 \\
3200 \\
2500\end{array}$ & $\begin{array}{l}3500 \\
3600 \\
3300\end{array}$ & $\begin{array}{l}3856 \\
3856 \\
3856\end{array}$ \\
\hline SKAGIT & $\begin{array}{l}\text { VITAL } \\
\text { RATIO } \\
\text { COMPONENT II }\end{array}$ & $\begin{array}{l}55700 \\
52000 \\
47800\end{array}$ & $\begin{array}{l}57800 \\
54200 \\
52500\end{array}$ & $\begin{array}{l}52381 \\
52381 \\
52381\end{array}$ \\
\hline SKAMANIA & $\begin{array}{l}\text { VITAL } \\
\text { RATIO } \\
\text { COMPONENT II }\end{array}$ & $\begin{array}{l}4700 \\
5400 \\
6200\end{array}$ & $\begin{array}{l}5300 \\
5600 \\
6400\end{array}$ & $\begin{array}{l}5845 \\
5845 \\
5845\end{array}$ \\
\hline SNOHOMISH & $\begin{array}{l}\text { VITAL } \\
\text { RATIO } \\
\text { COMPONENT I I }\end{array}$ & $\begin{array}{l}229700 \\
233800 \\
239900\end{array}$ & $\begin{array}{l}245800 \\
248900 \\
267500\end{array}$ & $\begin{array}{l}265236 \\
265236 \\
265236\end{array}$ \\
\hline SPOKANE & $\begin{array}{l}\text { VITAL } \\
\text { RATIO } \\
\text { COMPONENT II }\end{array}$ & $\begin{array}{l}284800 \\
279800 \\
260800\end{array}$ & $\begin{array}{l}289900 \\
285700 \\
286600\end{array}$ & $\begin{array}{l}287487 \\
287487 \\
287487\end{array}$ \\
\hline STEVENS & $\begin{array}{l}\text { VITAL } \\
\text { RATIO } \\
\text { COMPONENT II }\end{array}$ & $\begin{array}{l}16200 \\
16600 \\
14500\end{array}$ & $\begin{array}{l}16700 \\
17400 \\
16900\end{array}$ & $\begin{array}{l}17405 \\
17405 \\
17405\end{array}$ \\
\hline THURSTON & $\begin{array}{l}\text { VITAL } \\
\text { RATIO } \\
\text { COMPONENT II }\end{array}$ & $\begin{array}{l}73200 \\
70200 \\
68200\end{array}$ & $\begin{array}{l}74500 \\
74500 \\
76000\end{array}$ & $\begin{array}{l}76894 \\
76894 \\
76894\end{array}$ \\
\hline WAHK IAKUM & $\begin{array}{l}\text { VITAL } \\
\text { RATIO } \\
\text { COMPONENT II }\end{array}$ & $\begin{array}{l}3800 \\
3500 \\
3300\end{array}$ & $\begin{array}{l}4000 \\
3800 \\
3500\end{array}$ & $\begin{array}{l}3592 \\
3592 \\
3592\end{array}$ \\
\hline WALLA WALLA & $\begin{array}{l}\text { VITAL } \\
\text { RATIO } \\
\text { COMPONENT II }\end{array}$ & $\begin{array}{l}44500 \\
42900 \\
38200\end{array}$ & $\begin{array}{l}44800 \\
42500 \\
43000\end{array}$ & $\begin{array}{l}42176 \\
42176 \\
42176\end{array}$ \\
\hline
\end{tabular}


IABLE 4e. (contd)

\begin{tabular}{|c|c|c|c|c|}
\hline & & 1968 & 1969 & $1970^{(b)}$ \\
\hline WHATCOM & $\begin{array}{l}\text { VITAL } \\
\text { RATI0 } \\
\text { COMPONENT II }\end{array}$ & $\begin{array}{l}79500 \\
77000 \\
72600\end{array}$ & $\begin{array}{r}84500 \\
81300 \\
79300\end{array}$ & $\begin{array}{l}81950 \\
81950 \\
81950\end{array}$ \\
\hline WHITMAN & $\begin{array}{l}\text { VITAL } \\
\text { RATIO } \\
\text { COMPONENT II }\end{array}$ & $\begin{array}{l}29800 \\
34500 \\
35700\end{array}$ & $\begin{array}{l}30800 \\
34700 \\
38200\end{array}$ & $\begin{array}{l}37900 \\
37900 \\
37900\end{array}$ \\
\hline YAKIMA & $\begin{array}{l}\text { VITAL } \\
\text { RATIO } \\
\text { COMPONENT II }\end{array}$ & $\begin{array}{l}159800 \\
148300 \\
134500\end{array}$ & $\begin{array}{l}157800 \\
146400 \\
146400\end{array}$ & $\begin{array}{l}144971 \\
144971 \\
144971\end{array}$ \\
\hline
\end{tabular}
(a) Intercensus estimates are rounded to nearest hundred. (b) Actual census count.
(c) Average of birth and death estimates. 


\section{SUBCOUNTY HEDR POPULATION ESTIMATES}

Population estimates for each county census division in the HEDR study area are given for each year by county in Tables 5 a through $5 j$.

TABLE 5a. Adams County Population by Division

\begin{tabular}{|c|c|c|c|c|c|c|c|c|c|c|c|}
\hline 1940 & 1941 & 1942 & 1943 & 1944 & 1945 & 1946 & 1947 & 1948 & 1949 & 1950 & 1951 \\
\hline 40 & 40 & 42 & 40 & 42 & 41 & 43 & 39 & 41 & 42 & 42 & 39 \\
\hline 2974 & 3020 & 3170 & 2998 & 3180 & 3050 & 3204 & 2939 & 3063 & 3126 & 3154 & 2992 \\
\hline 135 & 137 & 144 & 136 & 144 & 138 & 145 & 133 & 139 & 142 & 143 & 136 \\
\hline 157 & 160 & 168 & 159 & 168 & 161 & 170 & 156 & 162 & 166 & 167 & 163 \\
\hline 1145 & 1163 & 1220 & 1154 & 1224 & 1174 & 1233 & 1431 & 1179 & 1203 & 1214 & 1147 \\
\hline 98 & 100 & 105 & 99 & 105 & 101 & 106 & 97 & 101 & 103 & 104 & 97 \\
\hline 270 & 274 & 287 & 272 & 288 & $2 \pi$ & 291 & 267 & 278 & 283 & 286 & 271 \\
\hline 620 & 629 & 660 & 624 & 662 & 635 & 667 & 612 & 638 & 651 & 657 & 623 \\
\hline 117 & 119 & 125 & 118 & 125 & 120 & 126 & 916 & 120 & 123 & 124 & 120 \\
\hline 654 & 664 & 696 & 659 & 699 & 670 & 704 & 646 & 673 & 687 & 693 & 887 \\
\hline 6209 & 6305 & 6617 & 6258 & 6639 & 6367 & $\triangle 688$ & 6136 & 6395 & 6525 & 6584 & 6474 \\
\hline 1952 & 1953 & 1954 & 1955 & 1956 & 1957 & 1958 & 1959 & 1960 & 1961 & 1962 & 1963 \\
\hline 39 & 39 & 40 & 41 & 40 & 38 & 35 & 33 & 30 & 30 & 31 & 29 \\
\hline 3051 & 3146 & 3268 & 3410 & 3423 & 3382 & 3302 & 3184 & 3075 & 3109 & 3179 & 2970 \\
\hline 139 & 144 & 150 & 157 & 158 & 157 & 154 & 149 & 145 & 144 & 145 & 132 \\
\hline 170 & 181 & 193 & 208 & 216 & 221 & 224 & 224 & 226 & 230 & 238 & 224 \\
\hline 1165 & 1196 & 1237 & 1284 & 1282 & 1258 & 1220 & 1168 & 1118 & 1135 & 1165 & 1093 \\
\hline 97 & 98 & 99 & 101 & 98 & 94 & 88 & 82 & 75 & 76 & 78 & 74 \\
\hline 275 & 283 & 293 & 305 & 305 & 300 & 292 & 280 & 269 & 267 & 267 & 244 \\
\hline 635 & 654 & 679 & 708 & 711 & 701 & 684 & 659 & 636 & 648 & 668 & 630 \\
\hline 125 & 132 & 140 & 150 & 154 & 157 & 158 & $\uparrow 57$ & 157 & 158 & 161 & 150 \\
\hline 1157 & 1473 & 1846 & 2283 & 2682 & 3072 & 3453 & 3812 & 4198 & 4553 & 4991 & 4998 \\
\hline 685 & 7346 & 7945 & 8646 & 9069 & 9380 & 9609 & 9747 & 9929 & 10352 & 10924 & 10545 \\
\hline
\end{tabular}

$1964 . \quad \underline{1965} \quad \underline{1966} \quad \underline{1967} \quad \underline{1968} \quad \underline{1969} \quad \underline{1970}$

$\begin{array}{rrrrrrrr}\text { AD1 } & 29 & 28 & 28 & 27 & 27 & 25 & 27 \\ \text { AD2 } & 2915 & 2746 & 2745 & 2659 & 2608 & 2449 & 2599 \\ \text { AD3 } & 127 & 117 & 113 & 107 & 101 & 91 & 93 \\ \text { AD4 } & 222 & 211 & 214 & 209 & 208 & 198 & 213 \\ \text { AD5 } & 1077 & 1020 & 1024 & 998 & 985 & 930 & 994 \\ \text { AD6 } & 73 & 69 & 69 & 68 & 67 & 63 & 68 \\ \text { AD7 } & 234 & 215 & 209 & 196 & 185 & 167 & 169 \\ \text { AD8 } & 623 & 593 & 599 & 586 & 582 & 553 & 595 \\ \text { AD9 } & 147 & 138 & 138 & 133 & 130 & 121 & 128 \\ \text { AD10 } & 5254 & 5304 & 5682 & 5899 & 6206 & 6254 & 7128 \\ \text { COUNTY } & & & & & & & \\ \text { ESTIMATE } & 10701 & 10440 & 10820 & 10881 & 11098 & 10853 & 12014\end{array}$


TABLE 5b. Benton County Population by Division

\begin{tabular}{|c|c|c|c|c|c|c|c|c|c|c|c|c|}
\hline & 1940 & 1941 & 1942 & 1943 & 1944 & 1945 & 1946 & 9947 & 1948 & 1949 & 1950 & 1951 \\
\hline BE1 & 968 & 972 & 992 & $16 \sqrt{5}$ & 1775 & 695 & 791 & 981 & 1157 & 1255 & 1289 & 1476 \\
\hline BE2 & 1000 & 1000 & 1000 & 4414 & 34315 & 2660 & 58 & 72 & 84 & 92 & 94 & 104 \\
\hline $\mathrm{BE3}$ & 3895 & 3830 & 3900 & 7493 & 15688 & 7242 & 8244 & 10220 & 12056 & 13076 & 13424 & 15697 \\
\hline BE4 & 1994 & 1918 & 1141 & 1973 & 2634 &, 1030 & 1173 & 1454 & $17 \uparrow 5$ & 1860 & 1910 & 2281 \\
\hline BE5 & 1686 & 1692 & 1725 & 3141 & 6006 & 2345 & 2670 & 3309 & 3904 & 4235 & 4347 & 4945 \\
\hline BE6 & 1284 & 1290 & 1315 & 2321 & 3627 & 1422 & 1619 & 2007 & 2367 & 2568 & 2636 & 2954 \\
\hline BE7 & 240 & 240 & 240 & 240 & 3336 & 13517 & 16746 & 22069 & 38729 & 32253 & 30950 & 27880 \\
\hline $8 E 8$ & 833 & 837 & 855 & 1400 & 980 & 385 & 438 & 544 & 641 & 696 & 714 & 761 \\
\hline BE9 & 1113 & 1117 & 1140 & 1970 & 2627 & 1028 & 1970 & 1450 & 1710 & 1855 & 1905 & 2233 \\
\hline COUNTY & & & & & & & & & & & & \\
\hline TIMATE & 12053 & 12097 & 12308 & 24627 & 70987 & 30325 & 32908 & 42105 & 62365 & 57889 & 57267 & 58330 \\
\hline
\end{tabular}

* For the years 1944-\$950, BE2 poputation reflects the numer of construction workers.

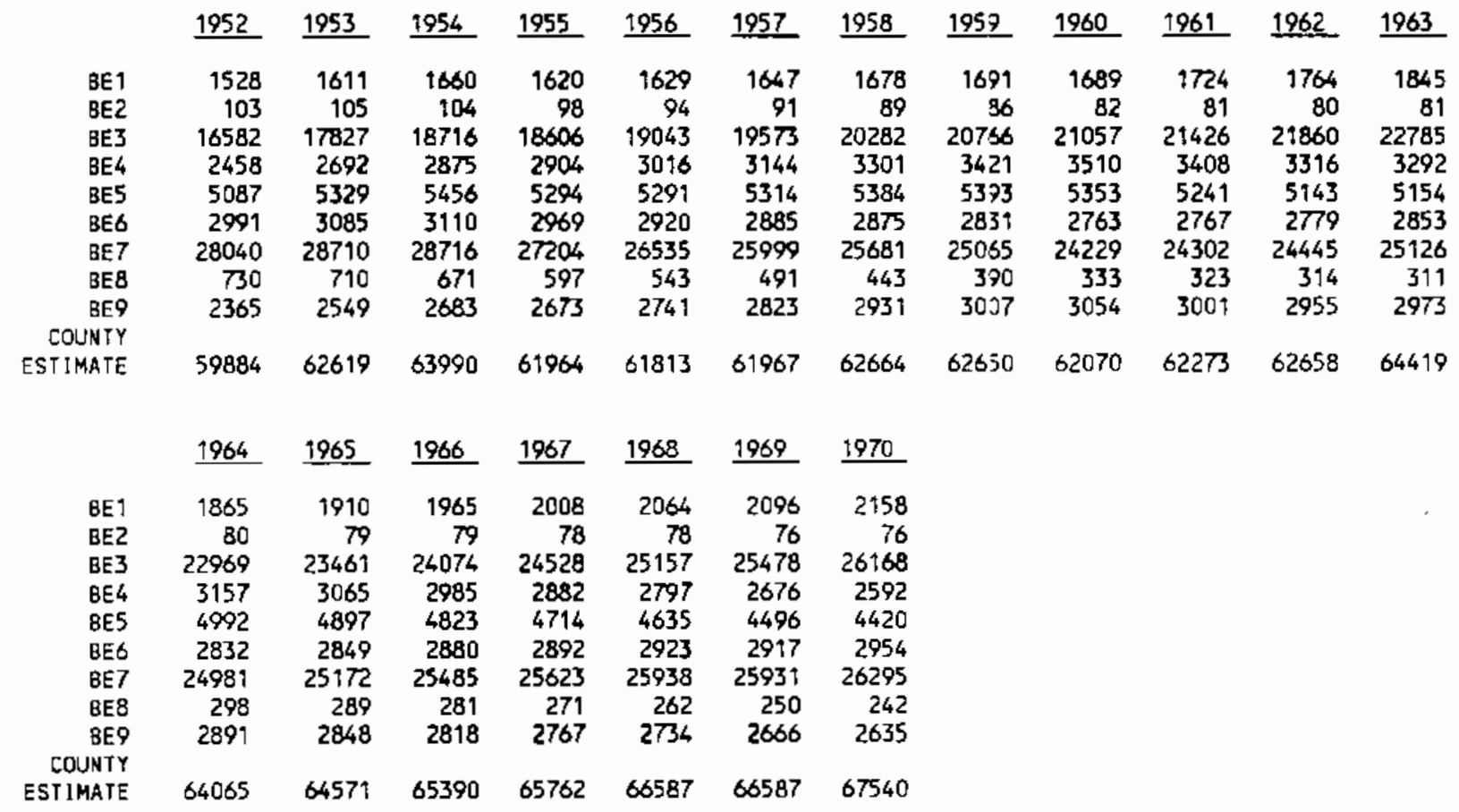


TABLE 5c. Franklin County Population by Division

\begin{tabular}{|c|c|c|c|c|c|c|c|c|c|c|c|c|}
\hline & 1940 & 1941 & 1942 & 1943 & 1944 & 1945 & 1946 & 1947 & 1948 & 1949 & $\underline{1950}$ & 1951 \\
\hline $\begin{array}{l}\text { FR1 } \\
\text { FR2 } \\
\text { FR3 } \\
\text { FR4 } \\
\text { FR5 }\end{array}$ & $\begin{array}{r}363 \\
338 \\
4756 \\
70 \\
780\end{array}$ & $\begin{array}{r}368 \\
342 \\
4819 \\
79 \\
790\end{array}$ & $\begin{array}{r}387 \\
359 \\
5063 \\
75 \\
830\end{array}$ & $\begin{array}{r}503 \\
467 \\
6585 \\
97 \\
1080\end{array}$ & $\begin{array}{r}544 \\
506 \\
7129 \\
105 \\
1169\end{array}$ & $\begin{array}{r}562 \\
522 \\
7357 \\
109 \\
1206\end{array}$ & $\begin{array}{r}582 \\
541 \\
7627 \\
143 \\
1251\end{array}$ & $\begin{array}{r}648 \\
603 \\
8491 \\
125 \\
1392\end{array}$ & $\begin{array}{r}683 \\
635 \\
8943 \\
932 \\
9466\end{array}$ & $\begin{array}{r}740 \\
688 \\
9696 \\
143 \\
1590\end{array}$ & $\begin{array}{r}781 \\
726 \\
10228 \\
151 \\
1677\end{array}$ & $\begin{array}{r}918 \\
756 \\
10953 \\
244 \\
1912\end{array}$ \\
\hline $\begin{array}{r}\text { COUNTY } \\
\text { ESTIMATE }\end{array}$ & 6307 & 6391 & 6713 & 8732 & 9454 & 9756 & 10114 & 11259 & 11859 & 12858 & 13563 & 14784 \\
\hline & $\underline{1952}$ & 1953 & 1954 & $\underline{1955}$ & 1956 & 1957 & 1958 & 1959 & 1960 & 1961 & 1962 & 1963 \\
\hline $\begin{array}{l}\text { FR1 } \\
\text { FR2 } \\
\text { FR3 } \\
\text { FR4 } \\
\text { FR5 }\end{array}$ & $\begin{array}{r}1018 \\
745 \\
11129 \\
335 \\
2066\end{array}$ & $\begin{array}{r}1167 \\
760 \\
11723 \\
448 \\
2310\end{array}$ & $\begin{array}{r}1289 \\
748 \\
11954 \\
557 \\
2497\end{array}$ & $\begin{array}{r}1538 \\
796 \\
13213 \\
731 \\
2922\end{array}$ & $\begin{array}{r}1669 \\
771 \\
13311 \\
857 \\
3114\end{array}$ & $\begin{array}{r}1853 \\
762 \\
13761 \\
1016 \\
3402\end{array}$ & $\begin{array}{r}1991 \\
728 \\
13794 \\
1154 \\
3601\end{array}$ & $\begin{array}{r}2163 \\
702 \\
13999 \\
1314 \\
3857\end{array}$ & $\begin{array}{r}2396 \\
687 \\
14522 \\
1518 \\
4219\end{array}$ & $\begin{array}{r}2522 \\
706 \\
15059 \\
1447 \\
4022\end{array}$ & $\begin{array}{r}2670 \\
730 \\
15718 \\
1383 \\
3843\end{array}$ & $\begin{array}{r}2910 \\
779 \\
16906 \\
1355 \\
3765\end{array}$ \\
\hline COUNTY & & & & & & & & & & & & \\
\hline ESTIMATE & 15294 & 16406 & $\uparrow 7045$ & 19202 & 19721 & 20795 & 21269 & 22035 & 23342 & 23756 & 24343 & 25714 \\
\hline & 1964 & 1965 & 1966 & 1967 & 1968 & 1969 & 1970 & & & & & \\
\hline $\begin{array}{l}\text { FR1 } \\
\text { FR2 } \\
\text { FR3 } \\
\text { FR4 } \\
\text { FR5 }\end{array}$ & $\begin{array}{r}2931 \\
768 \\
16809 \\
1220 \\
3390\end{array}$ & $\begin{array}{r}3012 \\
773 \\
17064 \\
1114 \\
3095\end{array}$ & $\begin{array}{r}3192 \\
803 \\
17876 \\
1041 \\
2892\end{array}$ & $\begin{array}{r}3212 \\
793 \\
17789 \\
914 \\
2541\end{array}$ & $\begin{array}{r}3269 \\
793 \\
17914 \\
802 \\
2230\end{array}$ & $\begin{array}{r}3516 \\
838 \\
19070 \\
733 \\
2036\end{array}$ & $\begin{array}{r}3556 \\
833 \\
19100 \\
616 \\
1711\end{array}$ & & & & & \\
\hline $\begin{array}{r}\text { COUNTY } \\
\text { ESTIMATE }\end{array}$ & 25117 & 25057 & 25804 & 25250 & 25009 & 26193 & 25816 & & & & & \\
\hline
\end{tabular}


IABLE 5d. Grant County Population by Division

\begin{tabular}{|c|c|c|c|c|c|c|c|c|c|c|c|c|}
\hline & 1940 & 1941 & 1942 & 1943 & 1964 & 1945 & 1946 & 1967 & 1948 & 1969 & 1950 & 1951 \\
\hline GR1 & 3236 & 2666 & 2814 & 2244 & 2154 & 2644 & 3302 & 3649 & 3744 & 4697 & 5371 & 5390 \\
\hline GR2 & 1036 & 854 & 901 & 719 & 690 & 847 & 1058 & 1169 & 1199 & 1504 & 1720 & 1763 \\
\hline GR3 & 124 & 102 & 107 & 86 & 82 & 101 & 126 & 139 & 143 & 179 & 205 & 219 \\
\hline GR4 & 542 & 447 & 472 & 376 & 361 & 443 & 553 & 611 & 627 & 787 & 900 & 938 \\
\hline GR5 & 1744 & 1436 & 1516 & 1209 & 1161 & 1425 & 1770 & 1966 & 2017 & 2531 & 2894 & 3060 \\
\hline GR6 & 848 & 698 & 737 & 588 & 564 & 693 & 865 & 956 & 981 & 1230 & 1407 & 1680 \\
\hline GR7 & 2942 & 2423 & 2559 & 2040 & 1958 & 2404 & 3002 & 3317 & 3404 & 4270 & 4883 & 5724 \\
\hline GRB & 774 & 637 & 673 & 536 & 515 & 632 & 789 & 872 & 895 & 1123 & 1284 & 1593 \\
\hline GR9 & 243 & 201 & 212 & 169 & 162 & 199 & 248 & 274 & 282 & 353 & 404 & 486 \\
\hline GR10 & 236 & 194 & 205 & 163 & 157 & 192 & 240 & 266 & 273 & 342 & 391 & 489 \\
\hline GR11 & 89 & 67 & 71 & 56 & 54 & 6 & 83 & 92 & 94 & 118 & 135 & 170 \\
\hline GR12 & 92 & $\pi$ & 80 & 64 & 61 & $\pi$ & 93 & 103 & 906 & 133 & 152 & 215 \\
\hline GR 13 & 7 & 5 & 6 & 5 & 4 & 5 & 7 & 7 & 8 & 10 & 11 & 44 \\
\hline GR14 & 2765 & 2277 & 2405 & 1917 & 1840 & 2259 & 2821 & 3118 & 3199 & 4013 & 4589 & 4900 \\
\hline COUNTY & & & & & & & & & & & & \\
\hline ESTIMATE & 14668 & 12083 & 12757 & $1017 !$ & 9763 & 11986 & 14969 & 16540 & 16972 & 21289 & 24346 & 26671 \\
\hline & 1952 & 1953 & 1954 & 1955 & 1858 & 1957 & 1958 & 1859 & 1960 & 1961 & 1962 & 1963 \\
\hline GR 1 & 4987 & 4993 & 4862 & 5119 & 4562 & 3840 & 3078 & 2336 & 1652 & 1801 & 1923 & 1953 \\
\hline GR2 & 1673 & 1726 & 1742 & 1918 & 1809 & 1641 & 1459 & 1302 & 1177 & 1212 & 1226 & 1182 \\
\hline GR3 & 217 & 236 & 252 & 295 & 299 & 294 & 287 & 286 & 295 & 303 & 305 & 293 \\
\hline GR4 & 906 & 954 & 987 & 1117 & 1089 & 1026 & 956 & 904 & 880 & 890 & 884 & 837 \\
\hline GR5 & 3003 & 3278 & 3391 & 3920 & 3911 & 3785 & 3632 & 3551 & 3593 & 3668 & 3678 & 3517 \\
\hline GR6 & 1851 & 2219 & 2605 & 3346 & 3702 & 3967 & 4212 & 4554 & 5096 & 5287 & 5387 & 5235 \\
\hline GR7 & 6211 & 7343 & 8518 & 10826 & 11865 & 12608 & 13282 & 14262 & 15858 & $163 \uparrow 2$ & 16480 & 15878 \\
\hline GR8 & 1811 & 2228 & 2675 & 3501 & 3939 & 4282 & 4605 & 5036 & 5693 & 5689 & 5578 & 5211 \\
\hline GR9 & 539 & 649 & 766 & 987 & 1096 & 1178 & 1254 & 1300 & 1525 & 1575 & 1598 & 1545 \\
\hline GR 10 & 559 & 691 & 833 & 1094 & 1233 & 1344 & 1449 & 1587 & 1797 & 1908 & 1988 & 1974 \\
\hline GRT & 196 & 244 & 296 & 390 & 442 & 483 & 522 & 573 & 650 & 704 & 746 & 753 \\
\hline GR12 & 269 & 355 & 449 & 614 & 716 & 802 & 885 & 989 & 1139 & 1297 & 1438 & 1508 \\
\hline GR13 & 77 & 122 & 173 & 256 & 316 & 370 & 423 & 487 & 574 & 564 & 543 & 497 \\
\hline GR14 & 4861 & 5269 & 5620 & 6582 & 6659 & 6543 & 6381 & 6350 & 6548 & 6672 & 6677 & 6371 \\
\hline COUNTY & & & & & & & & & & & & \\
\hline ESTIMATE & 27160 & 30247 & 33166 & 39964 & 41639 & 42165 & 42424 & 43596 & 46477 & 47882 & 48452 & 46756 \\
\hline & 1964 & 1965 & 1966 & 1967 & 1968 & 1969 & 1970 & & & & & \\
\hline GR1 & 1995 & 1926 & 1901 & 2020 & 2136 & 2250 & 2358 & & & & & \\
\hline GR2 & $\uparrow 150$ & 1059 & 1000 & 1018 & 1033 & 1046 & 1055 & & & & . & \\
\hline GR3 & 284 & 260 & 245 & 248 & 251 & 253 & 254 & & & & & \\
\hline GR4 & 799 & 722 & 669 & 667 & 664 & 658 & 650 & & & & & \\
\hline GR5 & 3391 & 3096 & 2897 & 2922 & 2939 & 2948 & 2947 & & & & & \\
\hline GR6 & 5130 & 4760 & 4527 & 4647 & 4745 & 4838 & 4916 & & & & & \\
\hline GR7 & 15428 & 14196 & 13386 & 13612 & 13801 & 13955 & 14065 & & & & & \\
\hline GRQ & 4903 & 4365 & 3977 & 3909 & 3811 & 3707 & 3588 & & & & & \\
\hline GR9 & 1508 & 1393 & 1319 & 1366 & 1371 & 1391 & 1608 & & & & & \\
\hline GR10 & 1975 & 1870 & 1813 & 1895 & 1972 & 2047 & 2116 & & & & & \\
\hline GR11 & 766 & 735 & 723 & 765 & 806 & 845 & 883 & & & & & \\
\hline GR12 & 1586 & 1570 & 1585 & 1718 & 1851 & 1982 & 2109 & & & & & \\
\hline GR 13 & 458 & 398 & 353 & 336 & 318 & 298 & 277 & & & & & \\
\hline GR 14 & 6130 & 5585 & 5214 & 5248 & 5266 & 5270 & 5255 & & & & & \\
\hline COUNTY & & & & & & & & & & & & \\
\hline ESTIMATE & 45502 & 41935 & 39607 & 40338 & 40964 & 41488 & 41881 & & & & & \\
\hline
\end{tabular}


IABLE 5e. Kittitas County Population by Division

\begin{tabular}{|c|c|c|c|c|c|c|c|c|c|c|c|c|}
\hline & 1940 & 1941 & 1942 & 1943 & 1944 & 1945 & 1946 & 1947 & 1948 & 1949 & $\underline{1950}$ & 1951 \\
\hline KII & 749 & 732 & 727 & 709 & 720 & 750 & 789 & 778 & 781 & 808 & 823 & 764 \\
\hline $\mathrm{K} 12$ & 350 & 342 & 340 & 332 & 337 & 351 & 369 & 364 & 365 & 378 & 385 & 351 \\
\hline KI3 & 115 & 112 & 111 & 109 & $\$ 10$ & 115 & 121 & 119 & 120 & 124 & 126 & 112 \\
\hline KI 14 & 28 & 28 & 27 & 27 & 27 & 28 & 30 & 29 & 29 & 30 & 31 & 29 \\
\hline K!5 & 4737 & 4631 & 4601 & 4485 & 4553 & 4748 & 4989 & 4922 & 4942 & 5112 & 5207 & 4750 \\
\hline$x 16$ & 679 & 663 & 659 & 643 & 652 & 680 & 715 & 705 & 708 & 732 & 746 & 678 \\
\hline$x ! 7$ & 662 & 647 & 643 & 627 & 637 & 664 & 698 & 688 & 691 & 715 & 728 & 670 \\
\hline$\times 18$ & 2276 & 2225 & 2211 & 2155 & 2188 & 2281 & 2397 & 2365 & 2375 & 2456 & 2502 & 2387 \\
\hline $\mathrm{k} 19$ & 1687 & 1649 & 1638 & 1597 & 1621 & 1691 & 1777 & 1753 & 1760 & 1820 & 1854 & 1709 \\
\hline KITO & 1276 & 1248 & 1240 & 1208 & 1227 & 1279 & 1344 & 1326 & 1332 & 137 & 1403 & 1295 \\
\hline KI१\} & 7670 & 7497 & 7448 & 7261 & 7372 & 7687 & 8078 & 7969 & 8002 & 8276 & 8430 & 7923 \\
\hline \multicolumn{13}{|l|}{ COUNTY } \\
\hline \multirow[t]{2}{*}{ ESTIMATE } & 20230 & 19775 & 19645 & 19153 & 19444 & 20275 & 21306 & 21018 & 21105 & 21829 & 22235 & 20668 \\
\hline & 1952 & 1953 & 1954 & $\underline{1955}$ & 1956 & $\underline{1957}$ & 1958 & 1959 & 1960 & 1961 & 1962 & 1963 \\
\hline KI1 & 744 & 742 & 738 & 727 & 727 & 717 & 734 & 729 & 750 & 773 & 750 & 719 \\
\hline KI2 & 334 & 327 & 318 & 307 & 300 & 290 & 290 & 281 & 282 & 295 & 291 & 284 \\
\hline $\mathrm{K} 13$ & 105 & 100 & 95 & 89 & 84 & 79 & 76 & 71 & 68 & 77 & 81 & 83 \\
\hline KI4 & 28 & 28 & 28 & 27 & 27 & 27 & 27 & 27 & 28 & 29 & 27 & 26 \\
\hline K15 & 4538 & 4447 & 4339 & 4191 & 4110 & 3972 & 3981 & 3870 & 3896 & 3922 & 3718 & 3478 \\
\hline KI6 & 646 & 631 & 613 & 590 & 577 & $55 \overline{5}$ & 554 & 536 & 537 & 549 & 528 & 502 \\
\hline$x 17$ & 646 & 639 & 630 & 615 & 609 & 595 & 603 & 593 & 604 & 633 & 625 & 609 \\
\hline K18 & 2384 & 2442 & 2490 & 2513 & 2575 & 2600 & 2723 & 2765 & 2908 & 2909 & 2739 & 2544 \\
\hline$K 19$ & 1651 & 1636 & 1615 & 1578 & 1567 & 1534 & 1557 & 1534 & 1566 & 1574 & 1489 & 1391 \\
\hline $\mathrm{K} ! 10$ & $\uparrow 253$ & 1243 & 1229 & $\{20\}$ & 1196 & 1172 & 1193 & 1177 & $\$ 203$ & 1238 & 1201 & 1150 \\
\hline$x \geq 11$ & 7803 & 7882 & 7931 & 7903 & 7997 & 7977 & 8257 & 8291 & 8625 & 9179 & 9199 & 9098 \\
\hline COUNTY & & & & & & & & & & & & \\
\hline \multirow[t]{2}{*}{ EST IMATE } & 20132 & 20118 & 20026 & 19744 & 19771 & 19518 & 19995 & 19873 & 20467 & 21177 & 20650 & 19885 \\
\hline & $\underline{1964}$ & 1965 & 1966 & 1967 & 1968 & $\underline{1969}$ & 1970 & & & & & \\
\hline KI1 & 690 & 714 & 745 & 731 & 772 & 779 & 879 & & & & & \\
\hline $\mathrm{K} 12$ & 277 & 291 & 308 & 307 & 330 & 338 & 387 & & & & & \\
\hline KI3 & 86 & 95 & 106 & $\$ 10$ & 123 & 131 & 156 & & & & & \\
\hline $\mathrm{KI} 4$ & 25 & 25 & 26 & 25 & 26 & 26 & 29 & & & & & \\
\hline KI5 & 3252 & $32 \pi 7$ & 3324 & 3171 & 3252 & 3180 & 3476 & & & & & \\
\hline $\mathrm{KI} 6$ & 478 & 490 & 506 & 492 & 515 & 514 & 575 & & & & & \\
\hline $\mathrm{KI} 7$ & 594 & 625 & 663 & 661 & 710 & 727 & 834 & & & & & \\
\hline K18 & 2360 & 2359 & 2371 & 2241 & 2273 & 2199 & 2375 & & & & & \\
\hline Ki9 & 1298 & 1305 & 1321 & 1257 & 1286 & 1254 & 1367 & & & & & \\
\hline K!10 & 1102 & 1139 & 1186 & 1163 & 1227 & 1236 & 1393 & & & & & \\
\hline KI11 & 8996 & 9591 & 10292 & 10396 & 11292 & 11701 & 13568 & & & & & \\
\hline \multicolumn{13}{|l|}{ COUNTY } \\
\hline EST IMATE & 19157 & 19912 & 20847 & 20555 & 21809 & 22085 & 25039 & & & & & \\
\hline
\end{tabular}


TABLE 5f. Klickitat County Population by Division

$1940 \quad 1941 \quad 1942 \quad 1943 \quad 1944 \quad 1945 \quad \underline{1946} \quad \underline{1947} \quad \underline{1948} \quad \underline{1949} \quad \underline{1950} \quad \underline{1951}$

\begin{tabular}{|c|c|c|c|c|c|c|c|c|c|c|c|c|}
\hline KL1 & 316 & 295 & 281 & 267 & 263 & 311 & 335 & 314 & 325 & 339 & 335 & 331 \\
\hline KL2 & 193 & 181 & 172 & 163 & 161 & 190 & 205 & 192 & 199 & 208 & 205 & 212 \\
\hline KL3 & 3250 & 3040 & 2889 & 2746 & 2709 & 3201 & 3444 & 3235 & 3350 & 3493 & 3448 & 3439 \\
\hline KL4 & $\$ 2$ & 11 & 11 & 10 & 10 & 12 & 13 & 12 & 13 & 13 & 13 & 20 \\
\hline KL5 & 600 & 562 & 534 & 507 & 500 & 591 & 636 & 598 & 619 & 645 & 637 & 628 \\
\hline KL6 & 532 & 497 & 473 & 449 & 443 & 524 & 563 & 529 & 548 & 571 & 564 & 557 \\
\hline KL7 & 3701 & 3462 & 3290 & 3128 & 3085 & 3646 & 3922 & 3685 & 3815 & 3979 & 3927 & 3877 \\
\hline KL8 & 876 & 819 & 778 & 740 & 730 & 862 & 928 & 872 & 903 & 941 & 929 & 911 \\
\hline KLQ & 874 & 817 & $\pi 7$ & 738 & 728 & 861 & 926 & 870 & 901 & 939 & 927 & 933 \\
\hline KL10 & 1003 & 938 & 892 & 847 & 836 & 988 & 1063 & 998 & 1034 & 1078 & 1064 & 1046 \\
\hline COUNTY & & & & & & & & & & & & \\
\hline \multirow[t]{2}{*}{ EST IMATE } & 11357 & 10622 & 10098 & 9596 & 9466 & 11186 & 12034 & 11306 & 11706 & 12208 & 12049 & 11952 \\
\hline & 1952 & 1953 & 1954 & 1955 & 1956 & 1957 & 1958 & $\underline{1959}$ & 1960 & 1961 & 1962 & 1963 \\
\hline KL1 & 333 & 342 & 355 & 369 & 349 & 336 & 331 & 340 & 354 & 351 & 353 & 356 \\
\hline KL2 & 223 & 239 & 258 & 279 & 274 & 274 & 281 & 299 & 322 & 309 & 301 & 293 \\
\hline$K L 3$ & 3506 & 3636 & 3822 & 4007 & 3832 & 3730 & 3721 & 3865 & 4064 & 4045 & 4080 & 4129 \\
\hline $\mathrm{KL} 4$ & 27 & 35 & 44 & 54 & 59 & 64 & 71 & 81 & 92 & 83 & 75 & 68 \\
\hline KL, & 632 & 648 & 672 & 696 & 658 & 632 & 623 & 639 & 664 & 655 & 655 & 657 \\
\hline KL6 & 562 & 576 & 600 & 622 & 589 & 567 & 560 & 576 & 599 & 594 & 597 & 602 \\
\hline KL7 & 3912 & 4016 & 4178 & 4336 & 4104 & 3954 & 3905 & 4015 & 4179 & 4192 & 4262 & 4347 \\
\hline KL8 & 912 & 930 & 961 & 989 & 930 & 889 & 874 & 888 & 917 & 943 & 921 & 933 \\
\hline$K: 9$ & 959 & 1004 & 1064 & 1125 & 1086 & 1064 & 1069 & 1119 & 1186 & 1193 & 1217 & 1245 \\
\hline$K L \uparrow 0$ & 1050 & 1073 & $111 \uparrow$ & 1147 & 1081 & 1036 & 1018 & 1041 & १०७८ & 1045 & 1025 & 1009 \\
\hline COUNTY & & & & & & & & & & & & \\
\hline \multirow[t]{2}{*}{ ESTIMATE } & 12116 & 12498 & 13065 & 13624 & 12958 & 12546 & 12449 & 12864 & 13455 & 13381 & 13485 & 13637 \\
\hline & 1964 & 1965 & 1966 & 1967 & $\underline{1968}$ & 1969 & 1970 & & & & & \\
\hline KLI & 351 & 354 & 358 & 314 & 319 & 310 & 310 & & & & & \\
\hline $\mathrm{K}: 2$ & 279 & 271 & 263 & 222 & 216 & 200 & 191 & & & & & \\
\hline KL3 & 4093 & 4142 & 4203 & 3702 & $37 / 5$ & 3677 & 3697 & & & & & \\
\hline$K L 4$ & 59 & 51 & 43 & 30 & 23 & 15 & 7 & & & & & \\
\hline KL5 & 645 & 647 & 650 & 568 & 573 & 553 & 551 & & & & & \\
\hline KL6 & 594 & 599 & 605 & 531 & 539 & 523 & 524 & & & & & \\
\hline $\mathrm{KL} 7$ & 4342 & 4428 & 4527 & 4017 & 4127 & 4050 & 4101 & & & & & \\
\hline KL8 & 925 & 937 & 951 & 838 & 855 & 833 & 838 & & & & & \\
\hline KL9 & 1246 & 1275 & $\uparrow 307$ & 1163 & 1197 & 1178 & $\$ 196$ & & & & & \\
\hline KL 10 & 971 & 954 & 939 & 801 & 790 & 745 & 723 & & & & & \\
\hline COUNTY & & & & & & & & & & & & \\
\hline ESTIMATE & 13505 & 13657 & 13846 & 12184 & 12414 & 12083 & 12138 & & & & & \\
\hline
\end{tabular}


TABLE 5g. Walla Walla County Population by Division

\begin{tabular}{|c|c|c|c|c|c|c|c|c|c|c|c|c|}
\hline & 1940 & 1941 & 1942 & 1943 & 1944 & 1945 & 1946 & 1947 & 1948 & 1949 & 1950 & 1951 \\
\hline HA1 & 690 & 682 & 701 & 723 & 725 & 802 & 825 & 826 & 840 & 863 & 906 & 902 \\
\hline WA2 & 134 & 133 & 136 & 140 & 141 & 156 & 160 & 160 & 163 & 168 & 176 & 173 \\
\hline WA3 & 674 & $\infty 67$ & 685 & 706 & 708 & 784 & 806 & 807 & 821 & 843 & 885 & 898 \\
\hline HA4 & 612 & 606 & 622 & 641 & 643 & 712 & 732. & 733 & 746 & $7 \infty 6$ & 804 & 796 \\
\hline UA5 & 397 & 392 & 403 & 496 & 417 & 469 & 475 & 475 & 483 & 496 & 521 & 516 \\
\hline WAG & 1458 & 1443 & 1483 & 1528 & 1533 & 1697 & 1745 & 1747 & 1777 & $\uparrow 825$ & 1916 & 1898 \\
\hline WA 7 & 508 & 503 & 517 & 533 & 534 & 591 & 608 & 609 & 619 & 636 & 668 & 652 \\
\hline WAB & 2744 & 2716 & 2791 & 2876 & 2884 & 3192 & 3283 & 3287 & 3343 & 3433 & 3605 & 3662 \\
\hline WA9 & 2571 & 2545 & 2615 & 2695 & 2703 & 2991 & 3077 & 3080 & 3132 & 3217 & 3378 & 3341 \\
\hline HA10 & 2416 & 2391 & 2457 & 2532 & 2540 & 2810 & 2891 & 2894 & 2943 & 3023 & 3174 & 3232 \\
\hline HA911 & 18344 & 18156 & 18661 & 19226 & 19284 & 21341 & 21952 & 24979 & 22349 & 22952 & 24102 & 23963 \\
\hline \multicolumn{13}{|l|}{ COUNTY } \\
\hline \multirow[t]{2}{*}{ ESTIMATE } & 30547 & 30234 & 31074 & 32015 & $32 \uparrow 12$ & 35538 & 36554 & 36599 & 37216 & 38219 & 40135 & 40031 \\
\hline & 1952 & 1953 & 1954 & 1955 & 1956 & 1957 & 1958 & 1959 & 1960 & 1961 & 1962 & 1963 \\
\hline WA? & 902 & 919 & 908 & 913 & 919 & 926 & 935 & 921 & 932 & 902 & 907 & 872 \\
\hline WA2 & 170 & 169 & 166 & 164 & 162 & 160 & 159 & 154 & 153 & 147 & 147 & 141 \\
\hline WA3 & 915 & 941 & 955 & 977 & 9001 & 1026 & 1054 & 9057 & 1087 & 1083 & 1120 & 1109 \\
\hline HA4 & 792 & 794 & 788 & 787 & 787 & 788 & 791 & $7 \pi$ & 779 & 760 & 770 & 746 \\
\hline WA5 & 513 & 515 & 519 & 511 & 511 & 512 & 515 & 504 & 507 & 494 & 501 & 485 \\
\hline WAG & 1890 & 1898 & 1884 & 1883 & 1887 & 1891 & 1901 & 1863 & 1875 & 1829 & 1855 & 1799 \\
\hline WA7 & 639 & 631 & 696 & 605 & 596 & 586 & 578 & 556 & 548 & 540 & 552 & 547 \\
\hline WAB & 3739 & 3849 & 3915 & 4009 & 4114 & 4223 & 4344 & 4358 & 4489 & 4482 & 4650 & 4616 \\
\hline WA9 & 3323 & 3333 & 3303 & 3298 & 3299 & 3302 & 3313 & 3242 & 3258 & 3238 & 3343 & 3303 \\
\hline WA10 & 3307 & 3412 & 3477 & 3568 & 3668 & 3772 & 3888 & 3907 & 4031 & 4049 & 4226 & 4219 \\
\hline WA 11 & 23954 & 24155 & 24065 & 24153 & 24292 & 24449 & 24667 & 24279 & 24536 & 24135 & 24671 & 24129 \\
\hline COUNTY & & & & & & & & & & & & \\
\hline \multirow[t]{2}{*}{ EST TMATE } & 40143 & 40610 & 40589 & 40867 & 41236 & 41636 & 42145 & 41616 & 42195 & 41660 & 42744 & 41960 \\
\hline & 1964 & 1965 & 1966 & 1967 & 1968 & 1969 & 1970 & & & & & \\
\hline WA 1 & 881 & 823 & 837 & 810 & 798 & 773 & 749 & & & & & \\
\hline WA2 & 141 & 131 & 132 & 127 & 125 & 120 & 115 & & & & & \\
\hline WA3 & 1154 & 1111 & 1164 & 1161 & 1181 & 1180 & $118 ?$ & & & & & \\
\hline WA4 & 760 & 716 & 735 & 718 & 714 & 699 & 684 & & & & & \\
\hline WAS & 494 & 466 & 477 & 466 & 464 & 454 & 444 & & & & & \\
\hline WAG & 1833 & 1728 & 1772 & 1732 & 1725 & 1687 & 1653 & & & & & \\
\hline WA7 & 556 & 529 & 548 & 542 & 545 & 538 & 533 & & & & & \\
\hline WAB & 4814 & 4644 & 4877 & 4880 & 4973 & 4901 & 4995 & & & & & \\
\hline WA9 & 3430 & 3294 & 3444 & 3431 & 3482 & 3473 & 3469 & & & & & \\
\hline WA10 & 4425 & 4292 & 4531 & 4557 & 4667 & 4698 & 4734 & & & & & \\
\hline WA11 & 24800 & 23580 & 24408 & 24073 & 24185 & 23884 & 23619 & & & & & \\
\hline \multicolumn{13}{|l|}{ COUNTY } \\
\hline EST IMATE & 43289 & 41315 & 42927 & 42498 & 42858 & 42486 & 42176 & & & & & \\
\hline
\end{tabular}


TABLE 5h. Yakima County Population by Division

\begin{tabular}{|c|c|c|c|c|c|c|c|c|c|c|c|c|}
\hline & 1940 & 1941 & 1942 & 1943 & 1944 & 1945 & 1946 & 1947 & 1948 & 1949 & 3950 & 1951 \\
\hline YA1 & 2165 & 2110 & 2100 & 2099 & 2218 & 2451 & 2654 & 2715 & 2758 & 2746 & 2967 & 2968 \\
\hline YA2 & 8704 & 8483 & 8444 & 8439 & 8920 & 9853 & 10672 & 10918 & 11091 & 11041 & 11930 & 11715 \\
\hline YAB & 2303 & 2244 & 2234 & 2233 & 2360 & 2607 & 2823 & 2888 & 2934 & 2921 & $3 \uparrow 56$ & 3088 \\
\hline YA4 & 236 & 230 & 229 & 228 & 241 & 267 & 289 & 296 & 300 & 299 & 323 & 306 \\
\hline YA5 & 46170 & 45000 & 44794 & 44767 & 47316 & 52268 & 5661 & 57914 & 58834 & 58571 & 6328 & 62704 \\
\hline YAG & 2130 & 2076 & 2066 & 2065 & 2182 & 2411 & 2611 & 2671 & 2714 & 2702 & 2919 & 2842 \\
\hline YA7 & 22490 & 21920 & 21820 & 21806 & 23048 & 25460 & 27376 & 28210 & 28659 & 28530 & 30826 & 30371 \\
\hline YAB & 840 & 819 & 815 & 815 & 861 & 951 & 1031 & 1054 & 1071 & 1066 & 1152 & 1150 \\
\hline YAQ & 11548 & 11255 & 11204 & 11197 & 19834 & 13073 & 14159 & 14485 & 16715 & 14649 & 15828 & 15010 \\
\hline YA10 & 1536 & 1498 & $149:$ & 1490 & 1573 & 1739 & 1884 & 1927 & 1958 & 1949 & 2106 & 2090 \\
\hline YA11 & 899 & 876 & 872 & 872 & 921 & 1018 & 1102 & 1127 & 1145 & 1740 & 1232 & 1226 \\
\hline YA12 & 0 & 0 & 0 & 0 & 0 & 0 & 0 & 0 & 0 & 0 & 0 & 0 \\
\hline ב & & & & & & & & & & & & \\
\hline STIMATE & 99019 & 96509 & 96069 & 96011 & 101477 & 112096 & 121413 & 124207 & 126180 & 125615 & 135723 & 134371 \\
\hline
\end{tabular}

\begin{tabular}{|c|c|c|c|c|c|c|c|}
\hline & 1952 & 1953 & 1954 & 1955 & 1956 & 1957 & 1958 \\
\hline YA1 & 3002 & 2976 & 3053 & 3223 & 3271 & 3295 & 3404 \\
\hline YA2 & 11636 & 11324 & 11404 & 11821 & 11779 & 11652 & 11822 \\
\hline YAB & 3057 & 2964 & 2974 & 3072 & 3049 & 3004 & 3036 \\
\hline YA4 & 293 & 274 & 265 & 263 & 250 & 235 & 226 \\
\hline YA5 & 62851 & 61732 & 62745 & 65648 & 66029 & 65936 & 67337 \\
\hline YAB & 2798 & 2698 & 2692 & 2764 & 2727 & 2670 & 2681 \\
\hline YA7 & 30270 & 29562 & 29873 & 31078 & 31079 & 30855 & 31621 \\
\hline YAB & $\uparrow 161$ & 1149 & 1176 & 1240 & 1256 & 1263 & 1303 \\
\hline YAQ & 16175 & 16110 & 16601 & 17606 & 17945 & 18157 & 188417 \\
\hline YA10 & 2099 & 2065 & 2102 & 2204 & 2220 & 2221 & 2270 \\
\hline YA11 & 1234 & 1218 & 1243 & 1306 & 1319 & 1323 & 1361 \\
\hline YA1Z & 0 & 0 & 0 & 0 & 0 & 0 & 1) \\
\hline COUNTY & & & & & & & \\
\hline \multirow[t]{2}{*}{ ESTIMATE } & 134576 & 132073 & 134132 & 140223 & 140924 & 140611 & 143911] \\
\hline & 1964 & 1965 & 1966 & 1967 & 1968 & 1960 & 1970 \\
\hline YA1 & 3869 & 3887 & 4034 & 6070 & 4267 & 4299 & 4341) \\
\hline YA2 & 12067 & 11955 & 12214 & 12137 & 12536 & 12450 & 12394 \\
\hline YA3 & 3023 & 2983 & 3035 & 3004 & 3091 & 3058 & 3032 \\
\hline YA4 & 222 & 224 & 233 & 236 & 248 & 250 & 253 \\
\hline YA5 & 70138 & 69459 & 70926 & 70445 & 72731 & 72198 & 77840 \\
\hline YAS & 2655 & 2625 & 2676 & 2054 & 2736 & 2712 & $269 i_{i}$ \\
\hline YA7 & 30070 & 29253 & 29340 & 28619 & 29014 & 28277 & $2762^{\prime} 1$ \\
\hline$Y A B$ & 1037 & 947 & 885 & 799 & 743 & 657 & 573 \\
\hline YAQ & 19477 & 19072 & 19317 & 19031 & 19489 & 19190 & 18941 \\
\hline YA10 & 2186 & 2120 & 2119 & 2059 & 2080 & 2020 & 1965 \\
\hline YA11 & 1367 & 1340 & 1354 & 1331 & 1360 & 1336 & 1316 \\
\hline YA12 & 0 & 0 & 0 & 0 & 0 & 0 & 1) \\
\hline \multicolumn{8}{|l|}{ COUNTY } \\
\hline EST IMATE & 146043 & 143865 & 146132 & 144385 & 148295 & $\$ 46446$ & 144971 \\
\hline
\end{tabular}


IABLE 5i. Morrow County Population by Division

\begin{tabular}{|c|c|c|c|c|c|c|c|c|c|c|c|}
\hline 1940 & 1941 & 1942 & 1943 & 1944 & 1945 & 1946 & 1947 & 1948 & 1949 & 1950 & 1951 \\
\hline $\begin{array}{r}1029 \\
2331 \\
977\end{array}$ & $\begin{array}{r}1020 \\
2311 \\
968\end{array}$ & $\begin{array}{r}997 \\
2258 \\
946\end{array}$ & $\begin{array}{r}973 \\
2204 \\
923\end{array}$ & $\begin{array}{r}949 \\
2150 \\
901\end{array}$ & $\begin{array}{r}949 \\
2150 \\
901\end{array}$ & $\begin{array}{r}997 \\
2258 \\
946\end{array}$ & $\begin{array}{r}949 \\
2150 \\
901\end{array}$ & $\begin{array}{r}1020 \\
2311 \\
968\end{array}$ & $\begin{array}{l}1115 \\
2526 \\
1058\end{array}$ & $\begin{array}{l}1135 \\
2571 \\
1077\end{array}$ & $\begin{array}{l}1138 \\
2570 \\
1092\end{array}$ \\
\hline 4337 & 4300 & 4200 & 4100 & 4000 & 4000 & 4200 & 4000 & 4300 & 4700 & 4783 & 4800 \\
\hline 1952 & 1953 & $\underline{1954}$ & $\underline{1955}$ & 1956 & 1957 & 1958 & 1959 & 1960 & 1961. & 1962 & 1963 \\
\hline $\begin{array}{l}1208 \\
2720 \\
1172\end{array}$ & $\begin{array}{l}1159 \\
2603 \\
1137\end{array}$ & $\begin{array}{l}1139 \\
2551 \\
1130\end{array}$ & $\begin{array}{l}1150 \\
2567 \\
1153\end{array}$ & $\begin{array}{l}1118 \\
2489 \\
1133\end{array}$ & $\begin{array}{l}1134 \\
2515 \\
1161\end{array}$ & $\begin{array}{l}1072 \\
2370 \\
1108\end{array}$ & $\begin{array}{l}1151 \\
2537 \\
1202\end{array}$ & $\begin{array}{l}1145 \\
2517 \\
1209\end{array}$ & $\begin{array}{l}1183 \\
2533 \\
1229\end{array}$ & $\begin{array}{l}1141 \\
2379 \\
1166\end{array}$ & $\begin{array}{l}1083 \\
2201 \\
1089\end{array}$ \\
\hline 5100 & 4900 & 4820 & 4870 & 4740 & 4810 & 4550 & 4890 & 4871 & 4945 & 4686 & 4373 \\
\hline 1964 & 1965 & 1966 & 1967 & 1968 & 1969 & 1970 & & & & & \\
\hline $\begin{array}{l}1177 \\
2332 \\
1166\end{array}$ & $\begin{array}{l}1216 \\
2348 \\
1186\end{array}$ & $\begin{array}{l}1197 \\
2253 \\
4150\end{array}$ & $\begin{array}{l}1209 \\
2218 \\
1143\end{array}$ & $\begin{array}{l}1228 \\
2198 \\
1145\end{array}$ & $\begin{array}{l}1255 \\
2191 \\
1154\end{array}$ & $\begin{array}{l}1237 \\
2107 \\
1121\end{array}$ & & & & & \\
\hline 4675 & 4750 & 4600 & 4570 & 4570 & 4600 & 4465 & & & & & \\
\hline
\end{tabular}


TABLE 5j. Umatilla County Population by Division

\begin{tabular}{|c|c|c|c|c|c|c|c|c|c|c|c|c|}
\hline & 1940 & 1941 & 1942 & 1943 & 1944 & 1945 & 1946 & 1947 & 1948 & 1949 & 1950 & 1951 \\
\hline UM1 & 1251 & 1259 & 1326 & 1389 & 1422 & 1427 & 1567 & 1667 & 1687 & 1980 & 2004 & 2044 \\
\hline UM2 & 1175 & 1183 & 1246 & 1305 & 1337 & 1341 & 1472 & 1567 & 1585 & 1860 & 1883 & 1899 \\
\hline UM3 & 4103 & 4130 & 4351 & 4556 & 4066 & 4682 & 5139 & 5470 & 5533 & 6495 & 6574 & 6837 \\
\hline UM4 & 1711 & 1722 . & 1814 & 1900 & 1946 & 1952 & 2143 & 2281 & 2307 & 2708 & 2741 & 2746 \\
\hline UM5 & 2071 & 2085 & 2196 & 2299 & 2355 & 2363 & 2594 & 2761 & 2793 & 3278 & 3318 & 3502 \\
\hline UMG & 7349 & 7397 & 7792 & 8159 & 8357 & 8385 & 9204 & 9797 & 9910 & 11632 & 117774 & 12412 \\
\hline UM7 & 996 & 1002 & 1056 & 1105 & 1132 & 1136 & 1247 & 1327 & 1342 & 1576 & 1595 & 1854 \\
\hline UMB & 614 & 618 & 651 & 682 & 698 & 701 & 769 & 819 & 828 & 972 & 984 & 1086 \\
\hline UM9 & 1028 & 1035 & 1090 & 1149 & 1169 & 1173 & 1287 & 1370 & 1386 & 1627 & 1647 & 1742 \\
\hline UM10 & 1560 & 1571 & 1655 & 1732 & 1774 & 1780 & 1954 & 2080 & 2104 & 2470 & 2500 & 2434 \\
\hline UP11 & 524 & 527 & 555 & 581 & 596 & 598 & 656 & 698 & 706 & 829 & 839 & 961 \\
\hline UM 12 & 1999 & 2012 & 2120 & 2220 & 2273 & 2281 & 2504 & 2665 & 2696 & 3164 & 3203 & 3445 \\
\hline $\operatorname{LM} \leqslant 3$ & 1003 & 1010 & 1064 & 1114 & 1141 & 1144 & 1256 & 1337 & 1353 & 1588 & 1607 & 1565 \\
\hline UN14 & 645 & 650 & 684 & 717 & 734 & 736 & 808 & 860 & 870 & 1022 & 1034 & 1074 \\
\hline COUNTY & & & & & & & & & & & & \\
\hline ESTIMATE & 26030 & 26200 & 27600 & 28900 & 29600 & 29700 & 32600 & 34700 & 35,100 & 41200 & 49703 & 43300 \\
\hline & 1952 & 1953 & 1954 & 1955 & 1956 & 1957 & 1958 & 1959 & 1960 & 1961 & 1962 & $1963^{\circ}$ \\
\hline UM1 & 2202 & 2167 & 1935 & 1909 & 1948 & 1848 & 1749 & 1811 & 1757 & 1757 & 1700 & 1668 \\
\hline UM2 & 2022 & 1965 & 1732 & 1685 & 1695 & 4583 & 1474 & 1500 & 1429 & 1480 & 1483 & 1505 \\
\hline UM3 & 7511 & 7339 & 6872 & 6919 & 7212 & 6987 & 6760 & 7155 & 7102 & 7321 & 7298 & 7376 \\
\hline UM4 & 2902 & 2798 & 2446 & 2358 & 2349 & 2171 & 1999 & 2009 & 1888 & 1840 & 1733 & 1654 \\
\hline UM5 & 3904 & 3974 & 3674 & 3750 & 3962 & 3890 & 3813 & 4088 & 4110 & 4139 & 4033 & 3986 \\
\hline UMG & 13820 & 14055 & 12978 & 13234 & 13967 & 13700 & 13417 & 14371 & 14434 & 14416 & 13927 & 13646 \\
\hline UM7 & 2251 & 2473 & 2449 & 2661 & 2976 & 3079 & 3168 & 3552 & 3723 & 3823 & 3797 & 3824 \\
\hline UMB & 1262 & 1335 & $\uparrow 280$ & 1351 & 4474 & 1491 & $i 503$ & 1655 & 1706 & 1728 & 1693 & 1682 \\
\hline UHA & 1947 & 1986 & 1840 & 1882 & 1992 & 1960 & 1925 & 2067 & 2082 & 2083 & 2017 & 1980 \\
\hline UHTO & 2493 & 2321 & 1950 & 1798 & 1702 & 1483 & 1275 & 1180 & 1004 & 995 & 954 & 927 \\
\hline UH११ & 1152 & 1253 & 1230 & 1327 & $\uparrow 474$ & 1517 & 1553 & 1734 & 1810 & 1823 & 1776 & 1755 \\
\hline UM12 & 3252 & 3060 & 2604 & 2436 & 2346 & 2086 & 1838 & 1756 & 1555 & 1571 & 1536 & 1522 \\
\hline UM13 & 1602 & 1491 & 1253 & 1155 & 1093 & 952 & 818 & 757 & 644 & 644 & 622 & 610 \\
\hline UM 14 & 9180 & 1183 & 1077 & 1084 & 1129 & 1093 & 1056 & 1177 & 1908 & 1110 & 1075 & 1056 \\
\hline COUNTY & & & & & & & & & & & & \\
\hline ESTIMATE & 47500 & 47600 & 43320 & 43550 & 45320 & 43840 & 42350 & 44750 & 44352 & 44726 & 43643 & 43193 \\
\hline & 1964 & 1965 & 1966 & 1967 & 1968 & 1969 & 1970 & & & & & \\
\hline UM1 & 1643 & 1636 & 9636 & 1633 & 9648 & 1661 & 1630 & & & & & \\
\hline UN2 & 1533 & 1577 & 1630 & 1680 & 1749 & 1820 & $\uparrow 84 \uparrow$ & & & & & \\
\hline UM3 & 7482 & 7666 & 7892 & 8102 & 8406 & 8794 & 8788 & & & & & \\
\hline UM4 & $\$ 582$ & 1527 & 1479 & 1427 & 1389 & 1348 & 1271 & & & & & \\
\hline UM5 & 3955 & 3966 & 3997 & $40 \$ 9$ & 4086 & 4152 & 4105 & & & & & \\
\hline UMG & 13423 & 13344 & 13330 & 13283 & 13382 & 13472 & 13197 & & & & & \\
\hline UM7 & 3865 & 3947 & 4050 & 4145 & 4288 & 4432 & 4457 & & & & & \\
\hline LMB & 1678 & 1692 & 1715 & 1734 & 1772 & 1811 & 1800 & & & & & \\
\hline UN9 & 1951 & 1944 & 1946 & 1943 & 1962 & 1979 & 1943 & & & & & \\
\hline UM 10 & 905 & 892 & 883 & 872 & 870 & 868 & 842 & & & & & \\
\hline UMT1 & 1742 & 1747 & 1761 & 1770 & 1800 & 1829 & 1808 & & & & & \\
\hline UM12 & 1515 & 1525 & 1541 & 1555 & 1586 & 1616 & 1603 & & & & & \\
\hline UM13 & 601 & 598 & 598 & 596 & $60 \%$ & 606 & 594 & & & & & \\
\hline UM14 & 1042 & 1039 & 1041 & 1041 & 1052 & 1062 & 1044 & & & & & \\
\hline COUNTY & & & & & & & & & & & & \\
\hline ESTIMATE & 42917 & 43100 & 43500 & 43800 & 44590 & 45370 & 44923 & & & & & \\
\hline
\end{tabular}




\section{REFERENCES}

Beck, D. M., et al. 1990. Milk Cow Feed Intake and Milk Production and Distribution Estimates for Phase I. PNL-7227 HEDR, Pacific Northwest Laboratory, Richland, Washington.

Berry, W. D., and Feldman, S. 1985. Multiple Regression in Practice. In Series: Quantitative Applications in the Social Sciences, Sage Publications, Beverly Hills, California.

Namboodiri, N. K. 1972. "Dn the Ratio-Correlation and Related Methods of Subnational Population Estimation. Demography. 9(3):446.

New York State Department of Commerce. 1979. Component Method II Computational Procedures for Estimating the Population of Counties. Albany, New York.

Pittenger, D. B. 1991. Letter Report: Population Estimates By Age, Sex and Race for 10-County Study Area. PNL-7931 HEDR, Pacific Northwest Laboratory, Richland, Washington.

Rives, N. W., and W. J. Serow. 1984. Introduction to Applied Demography. Data Sources and Estimation Techniques. Sage Publications, Beverly Hills, California.

Schmitt, R. C., and A. H. Crosetti. 1954. "Accuracy of the Ratio-Correlation Method for Estimating Postcensal Population." Land Economics, 30:279-281.

Shryock, H. S., and J. S. Siegel. 1976. The Methods and Materials of Demography. Academic Press, New York.

Swanson, D. A. 1980. "Improving Accuracy in Multiple Regression Estimates of Populating Using Principles from Casual Modelling." Demography. 17(4):3.

U.S. Bureau of the Census. 1941. U.S. Census of the Population: 1940. U.S. Government Printing Office, Washington, D.C.

U.S. Bureau of the Census. 1951. U.S. Census of the Population: 1950. U.S. Government Printing office, Washington, D.C.

U.S. Bureau of the Census. 1961. U.S. Census of the Population: 1960. U.S. Government Printing Office, Hashington, D.C.

U.S. Bureau of the Census. 1971. U.S. Census of the Population: 1970. U.S. Government Printing Office, Washington, D.C.

U.S. Bureau of the Census. 1978. Calculation of Rates and Factors in the Component Method II. Memorandum to Federal State Cooperative Program participants dated September 28, 1978, Bureau of the Census, Washington, D.C. 

APPENDIX A

REVIEW OF RATIO-CORRELATION (REGRESSION)

MODELS AND RESULTS BY D. A. SWANSON 
Regression Model Review

In terms of the three models, fy find that insing the: sane four symptomatic variables fot hree distinct decennial period model offers several advantages. First, the ratiocorrelarion form appears to be preferred over cercain alternatives (i.e., the difference-correlation form) decause the estimates are not affected by the presence of zero values (Swanson, 1976). Second, the four variables selected are an optimal chcice in that they aze the only ones availabie foz eaci year besinning in 1940. Third, the accuracy of the annulal estimates for each intercensa: period is maximized by using three distinct models, one for each of the tiroe intezcensal periods. Jourth, by not adding different symptomatic indicatcrs as they became available over the years (e.g., registered automobiles, sales $i a x$ receipts, state ligucz store sajes, coverec employmert, marriages, marital dissolutions, etc.), the changes in model characteristics can be examined in a manner that enhances one's ability to detect cata input errors. This is not a minor issue. Tayman and shafer (19a2) have demonstrated that measurement errors affecting the input data have a fax greater potential for creating subsequent errors in the inal pcpulation estimates than does the "temporal instabiltty" of model coefficients. Eifth, this approach avoids the temptation of simply including all available symptomatic indicators in a given regression, which has been found to be even less accurate than using the average of univariate regressicns (Namboodizi and Lalu, 1971). Sixth, as has been noted elsewhere (National Fesearch Council, 1980: $10-12)$, it is generally not possible to produce a set of population estimares that will minimize all accuracy criteria simultaneously, so it is necessary to make choices among different estimation technigues: By using the same symptomatic variables, these choices and their subsequent problems can be minimized.

Taking into consideration the prececing comments, I find that each of the three regression models corforms to the standard citteria for evaluating ratio-correlation regression models. First, each of the three models appears to be adequate in terms of the standard statistical and demographic criteria for evaluating ratio-correiation models: the explained variance for each model exceeds $90 \%$ and the sum of the coefticients for each model is approximately 1.00. Second, following a verification of the input data, each model was replicated in a second construction =un.

In terms or criticisa, there are several issues worth considering. Eirst, it would be useful to concuct a resicual analysis. Given the high values of the 
coefficients of determination, Extyene speciffeatson"erto is not likely but it would be useful to have the added assurance of a verification from an analysis of residuals. It would also be useful to know if other ors assumpticns are violated. Second, it would be useful to state if each model is constructed from a data set conceptualized as a population or a sample. If the cormer is the case, then it would be appropriaze to einminate the stardarc errors and signif icance tests from Table 2 . If the latter is tine case, then it would be appropriate to examise the issue of collinearity (Belsley, Kuh, and Welsch, 1980). Third, it may be useful to consiaer the effect of changing census accuracy on the estimates. At the national level, the U.S. Bureau of the Census has found that net underccust erzor declined from 1940 to 1970 (Brown, 1970). The sensitivity of the models and their estimates to net underount erroz may be a factor to consider before placing final approva: on any pcpulation estimates.

\section{Population Estimates}

Following the verification of each of the models, pcpulation. estimates were developed for each year encing in "5: (1945, 1955, and 1965) in accordance with each model using the procedures outlined in the report. These procedures resulted in population estimates that, when rounded to the nearest hundred, matched those given in Appendix A of the report. Each county's intercensal estimates were also found to mezge with the decennial census figures for each county. Thus, it appears that the models given in Table 1 are producing the estimates in Appendix $A$.

As a critical comment, it would be useful to dispjay the state population figure for each year in Appencix A. Also, it would be useful to cite the source of the indepencentiy estimated state population figures for each non-census year.

General Comments on mis Report

In Appendix B, the report accurateiy describes the process of developing county population estimates using the ratiocorrelation method. You may want to consider moving Appendix B to the section of the report labelled "Explanation of the Ratio-Correlation Technique." A sew minor typographical and other errons are in the report. Those that I found are noted in the copy that I have enclosed. 


\section{References}

SeIsley, D.A., $\Xi$. Kuh, and R. E. Welsch

1980. Regzession Diagnostics: Identifying Infiuential Data and Scurces of Coilinearity. New Yorik: J.Wìey.

Brown, G.H.

1970. "Statement of Hcn. George H. Brown, Dinector, Bureau of the Census, Accompanied By Robert Drury and Ccnrad Maeuber." is Accuracy of 2970 Census Erumeration and Related Matters. Hearings Before the Subcommittee on Census and Staristics of the Comititee on Post office and Civil Service, :House of Representatives, 91 st Congress, 2nd Session, Serial No. 91-30

Namboodin:, N.K. and N.M. La]u

1971. "The Average of Several Simple Regressicn Estimates as an Alternative to the Multipie Regression Estimate in Postcensal and Intercensal Population Estimation: A Case Study." Rural Sociology 36(2): 137-194.

National Research Council

1980. Estimating Population and Income of Small Areas Washington, D.C.: National Academy Press.

Swanson, D.A.

1976. "An Evaluation of 'Ratio' and 'Difference' Regression Methods for Estimating Sma11, Highly Concentrated Populations: The Case of Ethnic Grcups." Review of Public Data Use 6: 18-27.

Tayman, I. and E. Shafer

2982. "The Impact of Terporal Instability and Measurement Error on the Accuracy of RatioCorrelation Population Estimation Models." Presented at the Annual Meeting of the Population Association of America, San Diego, Califoraia. 



\section{APPENDIX B}

\section{REVIEW OF HEDR MODELS AND RESULTS BY D. B. PITTENGER}


Review and Comments on Population Estimates Report for Hanford Environmental Dose Reconstruction Project Prepared for Battelle Pacific Northwest Laboratories
Richland, Washington

Donald B. Pittenger The Demographics Laboratory 2065 Lakemoor Drive, S.W. Olympia, Washington'98502 (206) 352-3039

April 28, 1989 
Aside from a little editing, the March, 1980 working report titled "Population Estimates for the Hanford Environmental Dose Reconstruction Project" needs little in the way of corrections.

However, there are several places where material needs to be added to make the report both complete and more defensible against legal or other challenge.

The points that I think need to be addressed are presented below.

\section{SELECTION OF ACTUAL ESTIMATES}

The writers present several series of estimates of total populations for counties in Washington state, but failed to produce one "final" series to be used for the balance of dosage study research. On Page 4 , they suggest that the "ratio-correlation" technique glelds the best result, but fail to commit themselves completely.

What is missing here is an analysis of the estimates produced by the various techniques.

If nothing else, they could have prepared a series of graphs--perhaps using a semi-log scale--for Benton and Franklin counties showing how the series compared. Just scanning the numerical output, I found the 1940-1950 period particularly interesting for Benton County.

Besides Benton and Franklin, they also could graph other counties that have experienced impacts from World War II or perhaps the construction of dams along the Columbia River between 1945 and 1970. Such counties might include Clark, Chelan, Douglas, Grant, Kıng, Kitsap, Island, and Okanogan.

From such graphs, it might be possible to get an impression as to which technique tends to handle sudden population changes best.

Another test is to compare the estimates against actual census counts. Besides the July 15, 1944 Benton County census, there were some county-wide censuses in Washington during the 1970s which might be used as a check if test estimates were prepared for that decade. (These censuses are: Cowlitz, September 13, 1973; San Juan, September 22 , 1975; Stevens, September 25, 1975; and Pend Oreille, October 31, 1978.) 
Instead of selecting a set of estimates resulting from rone methodology, there are other options. For example, the estimates could be averaged across all methods." "Or, averaging might be done using a judgmental we1ghting scheme (for Instance, ratio-corellation could have a weight of 0.5 , Component $1 I$ could be 0.35 , and vital rates 0.15 ).

ANONYMOUS STATE POPULATION CONTROL NUMBERS

Even though all the estimates were forced to sum to state population totals for each year, these totals are not reported. Furthermore, no source is given for the control totals.

These anonymous statewide data also come 1nto play in establish1ng statewide vital rates for the vital rates method (see last paragraph, p. 16).

Clearly, the source of these data must be identlfied and the numbers justified.

\section{MINOR CRITICISMS}

The two subjects above are considered highly important, and should be dealt with as soon as possible. Below are notes on lesser issues taken in the sequence found in the report.

\section{Rural Populations}

The U.S. Census Bureau tabulates county populations by "rural" and "rural-farm" status, so these might be as good a proxy for the likelihood of drinking m1lk from backyard cows as simple dispersion. (See Page 2, last paragraph.)

\section{Fix Equation}

The second equation on Page 3 should have "symptomatic variable" or some such label substitute for "population".

\section{Post-1970 Estimates}

Paragraph 2, Page 4 indicates that OFM estimates are used after 1970 . Although th1s may be reasonable, I think you need a stronger justification statement here to forestall 
criticism.

\section{Ratio-Correlat1on Variables}

The question of variables used in the ratio-correlation estimates is fairly important, and I think the decision to use the same set over the entire 1940-1970 perlod (last paragraph, Page 4) needs stronger justification.

I (and an expert hired by, say, opposition lawyers should there be lawsults concerning radiation doses), might argue that you should have chosen the sets of independent variables that yielded the best fit for each decade. After all, isn't the purpose of this project to' derive the best possible data that can reasonably gotten? Accepting only those data avallable for the 30-year perlod makes some sense for an academic exercise-but this is the real world.

Therefore, if the present symptomat1c data are to be used, I think it should be shown (through $r$-squares of regression fits for actual decade changes, etc.) that any improvement using other variables would be negligible.

\section{Over-65 Populations}

The treatment of the population over age 65 in the Component-II (C-II) method may require more thought.

The reason why over-65s are broken out is because net migration of the retired population can be in the opposite direction from net migration of the rest of the population. From the report, it seems that no explicit treatment for over-65s was made at all; this ralses questions as to how good the C-II estimates were.

Granted that Medicade data did not exist until the late 1960s. One way around this problem would be to estimate decade net migration rates for the over-65 population using a cohort-survival procedure, and use the following formula to derive net migration rates for intercensal years:

$$
\text { RATEYRn }=\left(((1+\text { DECADERATE }) * \star 1 / 10)^{\star \star} n\right)-1
$$

where ** denotes exponentiation.

Estimates of $\underline{\text { Births by }} \underline{\text { Race }}$

Estimating births by race (pp. 7-8) might be overkill, given the small non-white populations involved. The C-II method 
uses race because, in some parts"of the countryt thete are large black populations that havesigificanty bigher: mortality and fertility than the balapce of the poplation

On the other hand, if data might be orought into future litigation, perhaps it is best to follow the accepted C-II procedures as far as possible.

Please note that the equations at the top of Page 8 are not needed. Since the denominators are identical, the share can be computed as follows:

$$
.9585=8274 / 8632
$$

Also, the phasing in the last three sentences of the second paragraph is repetitious--just state fhat you used U.S. total and white life tables for the years covering the census dates.

\section{Group Quarters}

The 1940 census presents some definitional problems because it followed rules for treating college students that were changed in 1950; the 1950 procedures essentially have been followed ever since. My 1940 census file for Washington counties has no group quarters tabulation, and the report states (Page 9) that none was reported for Yakima county--so probably nothing much can be done to clean up this aspect of 1940-1950 change.

I'm not sure how college students were handled for the other two decades. The New York State report uses total students rather than just those students living in group quarters. I suppose this $1 \mathrm{~s}$ not a mistake, although it seems to be so at first glance. One can argue that not all 1n-migrating students live in dorms, so it is simplest to make all students (regardless of origin) honorary group quarters residents. Check with Dave Swanson or the Census Bureau on this point. In any case, state explicitly how student group quarters populations were defined in the estimation technique used.

\section{Grade School Adjustment Ratios}

Ratios used to estimate population from enrollment data (Page 10) are applied uniformly for approximately five years on either side of each census point. This can create a marked "stairstep" effect--particularly as shown between 1954 and 1955 for Yakima County. Why not interpolate between these ratios so that the result is a smoother (and 
probably more realistic) Intercensal progression (On the other hand, if the cause for a lurch lin the rat1o, can be . found and 1 ts date established, that Information spould be
lncorporated in the procedure.)

Use of Life Tables for Ent1re Decades.

A similar problem crops up on Page 12. The paragraph in the middle of the page states that life tables calculated around a census year are used for the for the balance of the decade.

This can be justified when making post-censal estimates; 1t's hard to make reliable sub-national life tables. This is why census-centered life tables are specified in the methodology.

But the Hanford study is an exercise in historical demography. Life tables for both ends of each decade in the study can be found. Therefore, it makes sense to interpolate between the endpoint life tables, since it is easy to do so and because the results are clearly more realistic.

\section{Benton County Construction Workers}

The report suggests (bottom of page 17) that recorded numbers of military personnel and construction workers for the period $1944-1950$ be added to the estimates resulting from the various techniques. A table presents these numbers.

It wight save the reader time and trouble if these additions were performed and the results displayed in a table and on a graph.

\section{NOTE ON ESTIMATES THEMSELVES}

Although a complete set of estimation series came with the report, these data are not easy to evaluate.

First, the data used to compute the estimates were not sent with the report, so it was not possible to confirm that the computations were done correctly--though I understand that this indeed was done by another authority.

Second, the data were not graphed, so it is difficult to digest the data and understand how the numbers generated by 
the various techniques correspond torone anothers

Ideally, the writers of the repor shoud hop prepared graphs for the study area coupties astwell as other counties of interest. Then they should have presented an analysis of what the data meant. And they should have drawn conclusions as to which single method was best or else proposed a weighting scheme for averaging the data from the various methods into a single set of estimates.

FINAL THOUGHTS

My present role in the HEDR project is to critique the work of Battelle staff, presumably with the goal of keeping the study results as defensible as reasonably possible. Since there is no sure way of determining bistorical populations in years where no censuses were taken, the final estimates can only be evaluated on the basis of appeals to logic or on the performance of techniques in other times and places. The role of Battelle staff is to make the strongest possible case for the selected technology, and my task is to evaluate their case.

Most of the work they have done up to this point is sound. But they must make their final technical decision. The bullet is not yet bitten.

And they must give their decsion proper supporting arguments before I can present my own final judgment. They must conpince me. Me and the lawyers and juries, if it comes to that. 


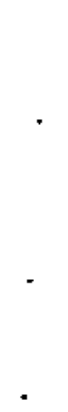




\section{APPENDIX C}

STEPS IN DEVELOPING RATIO-CORRELATION ESTIMATES 
APPENDIX C

\section{STEPS IN DEVELOPING RATIO-CORRELATION ESTIMATES}

Example Year 1941

Step 1: Construct Appropriate Regression Model for 1941-1949 Time Period a) Develop appropriate ratio of proportions for each symptomatic indicator. For example, for births the ratio is expressed

41

$$
\frac{\left(\frac{B i}{\sum_{B i}}\right) 1950}{\left(\frac{B i}{\Sigma B i}\right) 1940}
$$

where $B i=$ the number of births in county $i$

$\Sigma B i=$ the total number of births for all counties in the State

b) Regress the appropriate symptomatic indicator variables on the dependent variable. The dependent variable is the ratio of change in the proportion of each county population relative to the state population total. This can be symbolized as

$$
\begin{aligned}
\left\{\frac{\left(\frac{P i}{\sum P i}\right) 1950}{\left(\frac{P i}{\sum P i}\right) 1950}\right\} & =a+b 1\left\{\frac{\left(\frac{B i}{\sum B i}\right) 1950}{\left(\frac{B i}{\sum B i}\right) 1940}\right\}+b 2\left\{\frac{\left(\frac{D i}{\sum D i}\right) 1950}{\left(\frac{D i}{\sum D i}\right) 1940}\right\} \\
& +b 3\left\{\frac{\left(\frac{S E i}{\sum S E i}\right) 1950}{\left(\frac{S E i}{\sum S E i}\right) 1940}\right\}+b 4\left\{\frac{\left(\frac{R V i}{\sum R V i}\right) 1950}{\left(\frac{R V i}{\sum R V i}\right) 1940}\right\}
\end{aligned}
$$


where $P i=$ the census number in county $i$

$$
\begin{aligned}
& B i=\text { the number of births in county } i \\
& D i=\text { the number of deaths in county } i
\end{aligned}
$$

SE $i=$ the number of children enrolled in school in county $i$

RVi $=$ the number of registered voters in county $i$

$\Sigma P i=$ the census total for all counties in the State

$\Sigma B i=$ the total number of births for ail counties in the State

$\Sigma D i=$ the number of deaths for all counties in the State

$\Sigma S E=$ the number of children enrolled in school for all counties in the State

$\Sigma R V=$ the number of registered voters for all counties in the State

Step 2: Develop for each county and for each year an estimated ratio of population proportion. For 1941 the equation is

$$
\begin{aligned}
\frac{\left(\frac{P i}{\sum P i}\right) 1941}{\left(\frac{P i}{\sum P i}\right) 1940} & =.015+.153\left\{\frac{\left(\frac{B i}{\sum B i}\right) 1941}{\left(\frac{B i}{\sum B i}\right) 1940}\right\}-.035\left\{\frac{\left(\frac{D i}{\sum D i}\right) 1941}{\left(\frac{D i}{\Sigma D i}\right) 1940}\right\} \\
& +.366\left\{\frac{\left(\frac{S E i}{\sum S E i}\right) 1941}{\left(\frac{S E i}{\sum S E i}\right) 1940}\right\}+.500\left\{\frac{\left(\frac{R V i}{\sum R V i}\right) 1941}{\left(\frac{R V i}{\sum R V i}\right) 1940}\right\}
\end{aligned}
$$

Step 3: Produce unadjusted population estimates for each county and each year by taking the estimated ratio of population proportion for each county (derived in Step 2) and multiplying it by an independent state population total and by the county population proportion recorded in 1940 . 
Unadjusted Population Proportion $1941=$

$$
\frac{\left(\frac{I}{\sum P i}\right) 1941}{\left(\frac{P i}{\sum P i}\right) 1940} * \frac{P i}{\sum P i} 1940 *\left(\begin{array}{c}
\text { Independent } \\
\text { State } \\
\text { Population }
\end{array}\right)
$$

Estimate $\mathrm{a}^{(\mathrm{a})}$

Step 4: Produce adjusted county population estimates by dividing each county unadjusted population estimate by the sum of those estimates and then multiplying is number by the independent state population estimate for the estimate year

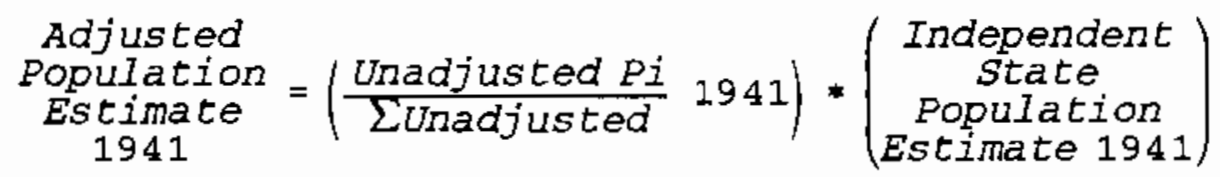

(a) Independent State Population Estimates were taken from official numbers provided by the U.S. Census. 


\section{REFERENCES}

United States Department of Commerce, Bureau of the Census, Population Division, Estimates of the Population of the States: 1940-1980, Washington, D.C. "State Ranking" File. 
APPENDIX D

USE OF COMPONENT METHOD II PRDCEDURE TO ESTIMATE 1947 YAKIMA COUNTY POPULATION 
APPENDIX D

\section{USE OF COMPONENT METHOD II PROCEDURE TO ESTIMATE 1947 YAKIMA COUNTY POPULATION}

The following paragraphs detaj] the component method II procedure using Yakima County data for estimating the 1947 Yakima County population. Annual estimates from 1940 to 1970 produced by this method are presented in the Results section of this report.

\section{PRELIMINARY DATA PREPARATION}

Calculating the expected population of school-age children requires single-year census population counts and vital statistics data broken down by race by county. This level of historical data was not available for the entire time-frame of the HEDR study for the entire study area.

Single-year census counts are required for the population age 6 to 14 , but are directly available for the 1960 and 1970 censuses only. For the 1940 and 1950 censuses, single-year census counts are obtained using Karup-King interpolation coefficients. An expansion of the theory behind this interpolation method is beyond the scope of this documentation, but for a reference please refer to Shyrock and Siegel (1976 p. 542 to 544).

Total statistics for the number of births and deaths by county for the 1940 to 1970 time frame were obtained. Racial breakdowns of these statistics were not readily available. The Vital Statistics of the United States ${ }^{(a)}$ was checked for the needed information. The data in these publications were tabulated in such a way that the actual number of white and nonwhite births and deaths for each county could not be ascertained. For this reason, the total number of births and deaths for the counties were allocated to white and nonwhite categories using fertility ratios based on the decennial censuses and national life tables.

(a) Published annually by the National Office of Vital Statistics, U.S. Department of Health, Education and Welfare, Public Health Service. 
The general fertility ratio of a county calculated from census data is the total number of children under 5 divided by the number of females of child bearing age, 15 to 44 (Shryock and Siegel 1976; p. 297). The general fertility ratio for a county is the ratio of the total number of white children (0 to 4) to the total number of child-bearing females (15 to 44) plus the ratio of the total number of nonwhite children to the total number of child-bearing females. Of total births, the percent of white births for a county is assumed to be the same as the percent that the white fertility ratio is of the total fertility ratio. The percent of white births is calculated for each census year according to Example 1 (for white births in Yakima County, 1940), and a linear interpolation is used to estimate the percent of white births for intercensal years.

The total number of deaths for a county is made up of white and nonwhite deaths. The number of white and nonwhite deaths for a census year can be estimated using national life tables and the census count. The percent of white deaths is calculated for the census years according to Example 2 (for white deaths in Yakima County 1940), and a linear interpolation is used to estimate the percent of white deaths for intercensal years. For 1940, 1939 to 1941 U.S. Life Tables for the total population and the white population are used. For 1950, 1949 to 1951 U.S. Life Tables for the total population and the white population are used. For 1960, 1959 to 1961 U.S. Life Tables for the total population and the white population are used. For 1970, the 1969 to 1971 U.S. Life Tables for the total population and the white population are used.

EXAMPLE 1. Calculating the Percent of White Births for 1940, Yakima County

$$
\begin{aligned}
\frac{(0-4 y r s \text { tot. })}{(15-44 \text { females })} & =\frac{(0-4 \text { yrs white })}{(15-44 \text { females })}+\frac{(0-4 \text { yrs nonwhts })}{(15-44 \text { females })} \\
\frac{8,632}{22,955} & =\frac{8,274}{22,955}+\frac{358}{22,955} \\
0.3760 & =0.3604+0.0156 \\
\frac{0.3604}{0.3760} & =\frac{\text { white fertility ratio of }}{\text { total fertility ratio }} \\
.9584 & =\text { percent of white births }
\end{aligned}
$$


EXAMPLE 2. Calculating Percent of white Deaths for 1940, Yakima County

(tota1 deaths) $=($ white deaths $)+($ nonwhite deaths $)$

(total pop.) $*$ (tot. death rt.) $=($ white pop. $) *($ white death rt.) + (nonwht pop.) * (nonwht death rt.)

$\frac{(\text { white pop. }) *(\text { white death rt.) }}{\text { (total pop.)*(tot. death rt.) }}=\frac{\text { white deaths }}{\text { total deaths }}$ $\frac{95399 *(0.01484)}{99019 *(0.01510)}=.9471=$ percent of white deaths

\section{HEDR COMPONENT METHOD II POPULATION ESTIMATES}

As already mentioned, the historical data available for HEDR population estimates varies from the data used by the U. S. Census Bureau for more recent estimations. The historical data obtained also varies across time periods. For this reason, the component method II methodology has been altered to accommodate the available data. The following paragraphs use data for Yakima County to estimate the 1947 population. It is pointed out where the component method II methodology has been altered to accommodate the available data.

1. Calculating the Non-Group Quarters Population Base (New York Department of Commerce 1979; p. 3)

Group quarter populations consist primarily of institutional inmates and college students living away from home. These numbers are reported as group quarter population in the decennial censuses. Intercensal estimates of group quarter populations are made by linearly interpolating the group quarters population documented in the two censuses that bracket the estimate year. These annual group quarter estimates are added to the annual non-group quarter population estimates.

The non-group quarters population base used to estimate an intercensal year population is the total population of a county in a census year minus the reported group-quarters population in that year. For example, the 1940 U.S. Census reports the population of Yakima County to be 99,019. It al so reports no group quarter population for 1940 . So the total non-group quarters population base used to produce a 1947 estimate is 99,019 . 
With in the HEDR study area, Benton County is an exception. Between the years of 1944 to 1950, Benton County experienced a large in-migration, and subsequently a large out-migration, of military and construction workers who were housed in group quarters. Since the migration occurred between census years, this population is not represented in the decennial censuses. The size of the group quarter population has been estimated through historical records.

2. Adjusting School Enrollment Data to Match the Census School-Age Population (New York Department of Commerce 1979; p. 7)

Since the enrollment for grades 1 to 8 rarely matches the census count for ages 6 to 14 in any given census year, the school enroliment numbers must be adjusted to match the census count for the appropriate ages. For the HEDR estimates from 1940 to 1967 , end-of-the-year public school enrollment numbers were available. For 1968 , fall public school enroll- . ment was available. For 1969 and 1970, combined public and private school fall enrollment numbers were available. The variety of data types available necessitates the use of slightly different formulas for these later years.

The annual enrollment numbers are first adjusted using the ratio of the ages 6 to 14 census count to the grades 1 to 8 enroliment for that census year. The relationship of the school enrollment data to the census count is used to adjust the enroliment data closest to that census year (1940 ratio is used to adjust the 1940 to 1944 enroliment; 1950 ratio is used to adjust the 1945 to 1954 enrollment data). Adjusting the enrollment data (end of year data or fall data) to match the census count (April) is implicit in this ratio method. Whether end of year data or fall data are used, the relationship of the census count to either type of enrollment data is maintained for similar enrollment data used in the annual estimates. No extra adjustment is made to adjust fall or end of year enrollment to April enrollment.

The annual enrollment numbers are then adjusted with an independent state control. This state control is the ratio of the state total for grades 1 to 8 to an independently derived estimate for the state's age 6 to 14 population (U.S. Census data). Two ratios are used for the 1970 data because both public enrollment and public with private enroliment data were available. Table D.1 shows how the state control adjustment ratios are calculated and for which years they are used.

Example 3 illustrates the process of adjusting the school enroliment data to match the census data. The procedure presented in Example 3, varies according to the type of school enrollment data that is available. For 1940 to 1964 , the procedure remains the same. For 1965 to 1967,1960 public end of school year ratios are used. For 1968, 1970 public fall ratios are used. For 1969 to 1970,1970 public-private fall ratios are used. 
EXAMPLE 3. Adjustment of School Enroliments for Yakima County, 1947

a. Population age 6.5 to 14.49 on

Apri $1,1950 \ldots \ldots . \ldots 1 . \ldots 284$

$0.75 \times($ no. age 6$)+($ no. age 7 to 13$)+$

$0.25 \times$ (no. age 14)

b. Number enrolled in 1950 (Grades 1 to 8).22,630

c. Ratio of school-age to enrollment $(\mathrm{a} / \mathrm{b})$. 0.8963

d. Number enrolled in $1947 \ldots . . . . .22,466$

e. Estimated school-age population in 1947 . 20,137 (c $\times d$ )

f. Adjustment of number derived in $c$ to independent state control

(Item c) $\times$ ratio of estimated state school-age population (which is the sum of the county school enroliments) to an independent Census Bureau state school-age population estimate (U.S. Census)

1950 Public Enrollment / age 6 - 14 census count 1.0933 (Varies depending on year of the estimate)

(c $\times 1.0933)$

g. Adjusted school enrollment for 1947 ( $f \times d$ ) 22,017

IABLE D.1. State Control Adjustment Ratios (for Yakima County 1947)

\begin{tabular}{|c|c|c|c|c|c|}
\hline Year & & $\begin{array}{c}\text { State } \\
\text { Enrollment }\end{array}$ & $\begin{array}{l}\text { State Census } \\
\text { Population }\end{array}$ & Ratio & $\begin{array}{l}\text { Ratio } \\
\text { Is Used }\end{array}$ \\
\hline $\begin{array}{l}1940 \\
1950 \\
1960 \\
1970\end{array}$ & $\begin{array}{l}\text { Publ ic } \\
\text { Publ ic } \\
\text { Public } \\
\text { Publ ic }\end{array}$ & $\begin{array}{l}215,352 \\
312,684 \\
433,435 \\
520,097\end{array}$ & $\begin{array}{l}195,123 \\
285,988 \\
465,244 \\
549,453\end{array}$ & $\begin{array}{l}1.10367 \\
1.09335 \\
0.93163 \\
0.94657\end{array}$ & $\begin{array}{r}1940-44 \\
1945-54 \\
1955-67 \\
1968\end{array}$ \\
\hline & Private & 555,206 & & 1.01047 & $1969-70$ \\
\hline
\end{tabular}


3. Expected Population of School-Age Assuming No Migration, 1947

(New York Department of Commerce $1979 ;$ p. 7-8)

The expected population of school-age children, assuming no migration, on April 1, 1947, is the cohort of the population 6.5 to 14.49 years old on the estimate date minus deaths to the cohort. The cohort is obtained from the appropriate age group in the 1940 Census. The cohort age group, the population age 6.5 to 14.49 on April 1, 1947, is the group 0 to 7.24 old on April 1, 1940, plus $3 / 4$ of the 1940 births. The number of survivors from this cohort is determined using national U.S. Life Tables, 1939 to 1941. Since the white and nonwhite populations have different death rates, the number of white survivors in this cohort was determined using national U.S. Life Tables for the white population, and the number of non-white survivors was determined using non-white life tables (Example 4).

The HEDR methodology differs from the component method II technique with respect to calculating the expected population. Where the HEDR method uses national mortality factors, the component method II uses agespecific death data for the county to calculate mortality factors. Agespecific death data were not found for the entire time frame of interest for the HEDR study.

EXAMPLE 4. Calculating the Expected School-Age Population for Yakima County, 1947

White Nonwhite

a. (no, age 0 to 6$)+0.25 \times($ no. age 7$)$ in 1940

b. Survival rate for age 0 to 7 from 1940-47 . . . . . . . . . . .

c. Number of age 0 to 7 survivors from $1940-47(a \times b)$

d. $3 / 4$ of 1940 births ........... 1,483

e. Survival rate of 1940 births to 1947 . . .

f. Number of 1940 birth survivors $(d \times$ e) . $\quad 1,455$

g. Expected population in $1947(c+f)$

13,285

578

h. Total expected population

(white + nonwhite) 
The 1 ife tables used to calculate deaths change from decade to decade. The U.S. Life Tables are published for the three-year period at the beginning of every decade, beginning with 1939 to 1941 . The 1 ife table used for a particular decade is the life table for the beginning of that decade. For the 1940 decade, the 1939 to 1941 national life tables are used. For the 1950 decade, the 1949 to 1951 national life tables are used. For the 1960 decade, 1959 to 1961 national life tables are used.

4. Net School-Age Migration from 1940 to 1947

(New York Department of Commerce 1979; p. 8-9)

The net migration from 1940 to 1947 is equal to the adjusted school enrollment for 1947 minus the expected enrollment for 1947 (Example 5).

EXAMPLE 5. Net Migration for the School-Age Population, Yakima County, 1947

a. 1947 adjusted school enroliment .... 22,017

b. 1947 total expected school enrollment . . 13,863

c. Net school-age migration, ...... 8, 154 $1940-47(a-b)$

5. School-Age Migration Rate, 1940 to 1947 (New York, 1979; p. 8-9)

The school-age migration rate is the net migrants divided by a schoolage population base. The school-age migration rate is calculated in Example 6 .

EXAMPLE 6. 1947 School-Age Migration Rate, Yakima County 1947

a. Net school-age migration, . . . . . 8, 154 $1940-47$ (7c)

b. Population base is equal to the 1940 school-age cohort plus $1 / 2$ the change between 1940 and 1947 . Births became part of the cohort in 1947. A factor to adjust for the different length of exposure for births versus the existing 1940 cohort is multiplied times the number of births and subtracted from the school-age base as follows: 
1. 1940 school-age cohort plus $3 / 41940$

births $14,012 \ldots . . . .$.

(Example 4: $a+d)$

14,012

2. 1/2 change in 1940 school-age cohort

$\{($ Example 4: $a+d)-($ Example 4: a) $\} \times 0.5$

3. $3 / 41940$ births ........ 1,547

4. Migration Exposure factor $=1 / 2 *$ [9 months + ( (est. yr beginning school age -1$) * 12\}]$ (est. yr * 12 months)

$$
\frac{1 / 2 *[9+((7-6-1) * 12\}]}{(7 * 12)} \cdot \cdot 0.0535714
$$

5. Population base ...... 14,003 $(b 1+b 2-\{b 4 * b 3\})$

c. School-age migration rate (a / b5) ... 0.5823

6. Converting School-Age Migration Rate into Population Migration Rate (U.S. Census Bureau Memorandum 1978; p.5)

The school-age migration rate is converted into a total population migration rate with the following formula:

School Migration Rate $+[(0.10 \times n) \times$ Residual Migration Factor $]$

where $n=$ the number of years between the 1940 census and the estimate date. The residual migration factor for a decade is the difference between the school-age migration rate for that decade and the under-65 female migration rate for that decade. County-specific residual migration factors for a specific decade are calculated as in Example 7. 
EXAMPLE 7. County-Specific Residua] Migration Adjustment Factor, Yakima County 1947

Mortality rates are calculated using national U. S. Life Tables.

\section{Whites}

Non-

Whites

a. School-age population, Apri1 1, 1950 . . . . . . 19,521

764

$(0.75 *$ no. age 6$)+$

(no. age 7 to 13$)+$

$(0.25 *$ no. age 14$)$

b. Population 0 to 4, Apri1 1, 1940 ....... 7,048

(no. age 0 to 3$)+(0.25 *$ no. age 4$)$

304

c. Sum of births from April 1, 1940 to

December 31,1943 . . . . . . . . . . 7,490

(use $3 / 4$ of 1940 births instead of

births by month)

d. Deaths to age $0-t_{0}-4$ cohort from 4-1-40 to

4-1-50 plus deaths to births (4-1-40 to

12-31-43). Total deaths to school-age

cohort are calculated using life table

survival rates (see Table D.2) . . . . . . . 254

e. Total school-age migration . . . . . . . . . . 5,386

$(a-b-c+d)$

f. Factor for underexposure of school-age

population to migration ........... 1.0963

g. Total School-age migration rate . . . . . . . 0.3892

$(e /\{b+c\}) \star f$

Non-

Whites Whites

h. Females under 65 years old,

April 1, 1950.............. 59,763

2,151

i. Females under 55 years old,

April 1,1940............... 39,499

j. Female births, April 1, 1940 -

March 31, 1950 ................ . 3,383

(3/4 41940 births $+1941-49$ births +

$1 / 4 \times 1950$ births) * (state $\%$ females)

k. Deaths to female 0 to 55 cohort and 1940-50

female births............... . . 1,812

(see Table D.3) 
1. Total migration for females under 65 on April 1, 1950............. 8, ... 806 $(h-i-j+k)$

m. Factor for underexposure of population under 65 to migration............. 1.0833

n. Total migration rate for females under 65 years on April 1, 1950 . . . . . . . . . 0.1755 $(1 /(i+j)) \star m$

0 . Residual migration adjustment factor

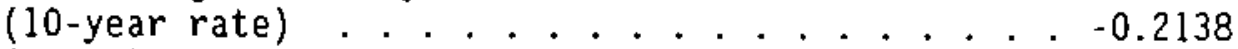
$(n-g)$

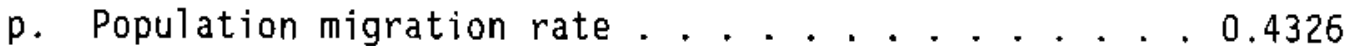

(school migration rate + $[(0.10 \times n) \times$ residual factor $)]$

\begin{tabular}{|c|c|c|c|c|c|c|}
\hline Cohort & $\begin{array}{l}\text { White } \\
\text { Pop } \\
\end{array}$ & $\begin{array}{l}\text { Death } \\
\text { Rate } \\
\end{array}$ & $\begin{array}{l}\text { White } \\
\text { Deaths }\end{array}$ & $\begin{array}{c}\text { Non-White } \\
\text { Pop }\end{array}$ & $\begin{array}{r}\text { Death } \\
\text { Rate } \\
\end{array}$ & $\begin{array}{l}\text { Non-White } \\
\text { Deaths }\end{array}$ \\
\hline $19400-4$ & $\overline{7,048}$ & $\overline{0.01365}$ & 96 & 304 & $\overline{0.02161}$ & 7 \\
\hline $\begin{array}{rl}3 / 4 & 1940 \\
1941 & \text { Births } \\
1942 & \text { Births } \\
1943 & \text { Births }\end{array}$ & $\begin{array}{l}1,483 \\
2,002 \\
1,902 \\
2,102\end{array}$ & $\begin{array}{l}0.02249 \\
0.02164 \\
0.02077 \\
0.01982\end{array}$ & $\begin{array}{l}33 \\
43 \\
39 \\
42\end{array}$ & $\begin{array}{l}64 \\
88 \\
84 \\
94\end{array}$ & $\begin{array}{l}0.03912 \\
0.03797 \\
0.03681 \\
0.03558\end{array}$ & $\begin{array}{l}3 \\
3 \\
3 \\
3\end{array}$ \\
\hline
\end{tabular}

IABLE D.3. Female Deaths

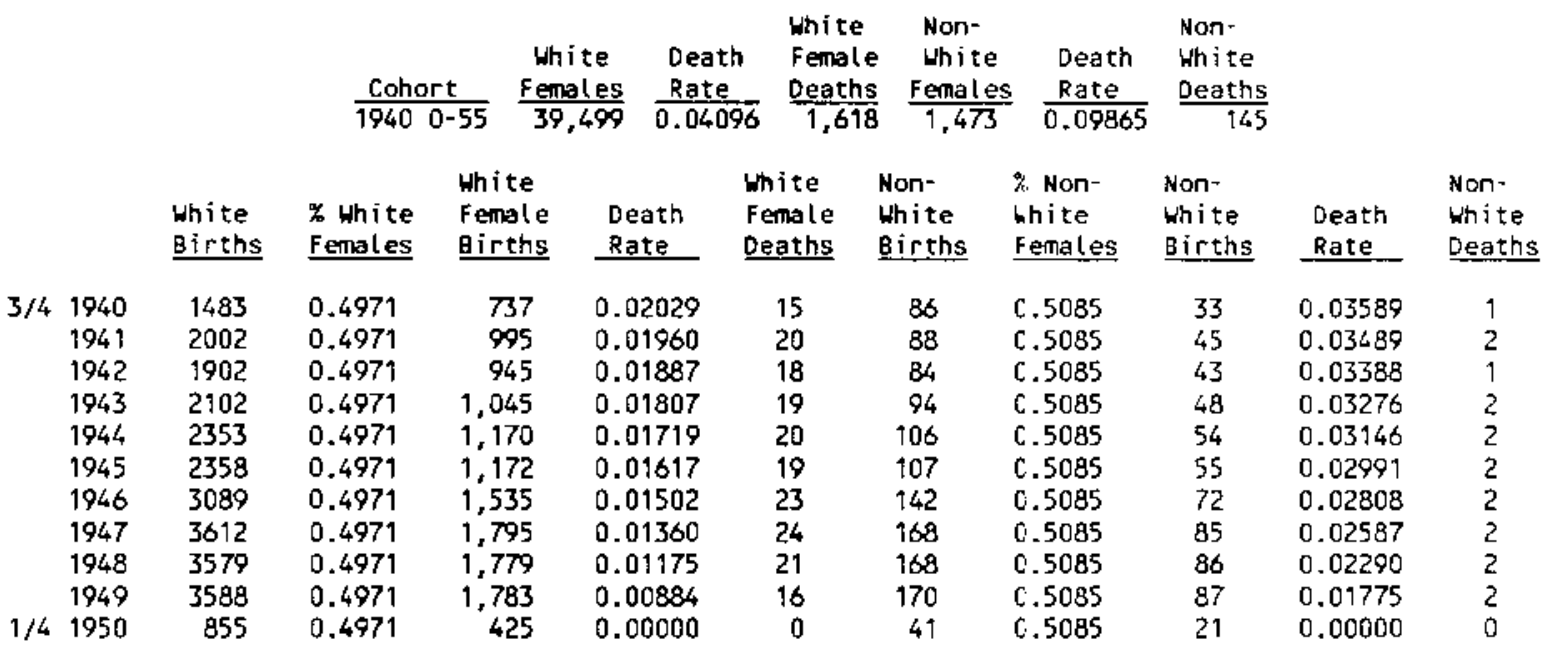


7. Estimatinq Total Population

(New York Department of Commerce 1979; p. 14)

The total population estimate is derived by adding the natural increase and the number of net migrants from the census to the estimate date to the census count (Example 8). The natural increase is the difference between, the births and deaths during the estimate period. The number of net migrants is calculated using the population migration rate that was calculated in the preceding section.

EXAMPLE 8. Preliminary Population Estimate for Yakima County, 1947

a. Non Group Quarters Population on April 1, $1940 \ldots . . . . .$. 99,019

b. $1 / 2$ the births, $1940-47 \ldots \ldots 8,8 . \ldots$

c. $1 / 2$ the deaths, $1940-47 \ldots \ldots 3,616$

d. Net migration base $(a+b-c) \quad \ldots . . \quad 103,863$

e. Net migrants $(d \times 7 p) \ldots \ldots 44,938$

f. Natural Increase, (1940-47 Births 1940-47 Deaths) ......... 9,687

g. 1947 Population $(a+e+f) \ldots \ldots 153,638$ 
8. Adjusting Population Estimate According to the Error of Closure

The error of closure is the percent difference between an estimated census year population and the census count. For each county, the error of closure is calculated and the error is linearly interpoTated and added to the intercensal estimates. The procedure illustrated above is used to calculate a census year estimate (1950). This 1950 estimate of the non-group quarters population is compared with the census count for the non-group quarters popula. tion in 1950. The difference distributed eventy to all of the inter-census estimates. Example 9 illustrates this procedure.

EXAMPLE 9. Final Population Estimate for Yakima County, 1947
a. 1950 estimated population
170,356
b. 1950 non-group quarters census coun
135,216
c. Difference $(b-a)$
$-35,140$
d. Annual increment (c/ 10)
$-3,514$
e. Preliminary 1947 non-group quarters ...
153,638
f. 1947 group quarters population
355
g. Final $1947(e+(7 * d))+f \ldots .$.
129,395 


\section{$\underline{\text { REFERENCES }}$}

Shyrock, H. S., and J. S. Siegel. 1976. The Methods and Materials of Demography. Academic Press, New York.

New York State Department of Commerce. 1979. Component Method II Computational Procedure for Estimating the Population of Counties. Albany, New York.

Bureau of the Census. 1978. Calculation of Rates and Factors in the Component Method II. Memorandum to Federal State Cooperative Program Participants dated September 28, 1978, Bureau of the Census, Washington, D.C.

U.S. Census Bureau Memorandum 1978 


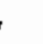




\section{APPENDIX E}

ESIIMATING COUNTY CENSUS DIVISION POPULATIONS 


\section{APPENDIX E}

\section{ESTIMATING COUNTY CENSUS DIVISION POPULATIONS}

To produce population estimates for county census divisions (CCDs), total county population estimates were disaggregated to their respective CCDs. This was done by interpolating the $C C D^{\prime}$ s proportional share of the county population recorded in the census years bracketing the estimate year. This procedure is symbolized as:

$$
P_{N}=[((Y-X) / 10\} * N]+X
$$

where $P_{N}=C C D$ 's share of county population for intercensal year $N$

$X=C C D^{\prime}$ s share of county population in earijer census year

$Y=C C D^{\prime}$ s share of county population in later census year

$N=$ Number of years from earlier census

The CCD's share of the county population for each intercensal year is then multiplied by the total population estimate for the relevant county (ratiocorrelation estimate) to produce an intercensus estimate. This interpolation technique was used for the years 1950 to 1970 .

For the 1940, decade CCD population shares were assumed to be equal to the shares reported in the 1950 census. This assumption was deemed necessary because of the radical demographic change that occurred from 1943 to 1950 as a result of World War II. Relatively large populations moved into lightly populated areas as the result of military base construction. This demographic change drastically altered the settlement patterns within the HEDR study area. As a result, we believe 1950 CCD population shares are more representative of the likely CCD population shares for the HEDR Phase I time span 1944 to 1950. Stated differently, population distribution in 1943 to 1945 was much more like that recorded in 1950 than the distribution recorded in the 1940 census.

CCD boundaries changed over the time period 1944 to 1970 for which estimates were prepared. To facilitate comparisons across decades, we standardized CCD boundaries to those reported in the 1970 census. This required the aggregation of some CCDs and parts of CCDs for the earlier decades. Table E.l 
indicates which CCDs for each county were combined to standardize all CCDs to the 1970 boundaries. For the purpose of the HEDR study, some of the CCD boundaries were modified into HEDR county divisions. Annual population estimates for each HEDR county division are reproduced in the Results section of this report. Age, sex, and race distributions for the HEDR county divisions are assumed to be identical to those reported for the county as a whole. 
TABLE E.1. Aggregation of CCD Boundaries to 1970

1970

\section{ADAMS COUNTY}

Division 1

Division 2

Division 3

Division 4

Division 5

Division 6

Division 7

Division 8

Division 9

Division 10

BENTON CDUNTY

Benton City Division

Federal Reservation Division

Kennewick Division

Kennewick South Division

Northwest Benton Division

Prosser Division

Richland Division

South Benton Division Division

West Richland

Division

FRANKLIN COUNTY

Connell Division

Kahlotus Division

Pasco Division

Pasco North Division

GRANT COUNTY

Division 1

Division 2

Division 4

Division 5

Division 7

Division 9

Division 10

Division 11

Division 12

Division 14

Division 15
1960

$1940 \& 1950$

Division 1

Division 2

Division 3

Division 4

Division 5

Division 6

Division 7

Division 8

Division 9

Division 10

.25 (Div. 12-BC-WR)+BC

Division 1

Kenn.+Div.17+.5(Div.16) +.5 (Div. 18)

Div.19+.5(Div.16)+ .5 (Div. 18)

Div. 13+Div. 14+.5(Div. 12 $-B C-W R$ )

Prosser

Rich? and \& Div. 2

Divisions $15 \& 20$

Div.11+WR+.25(Div.12$B C-W R$ )

Division 2

Divisions $1 \& 4$

Pasco

Division $3 \& 5$

Division 1

Division 2

Divisions $3 \& 4$

Divisions $5 \& 13$

Division 7

Division 8 \& 9

Division 10

Division 6 \& 11

Division 12

Division 14

Division 15
Division 1

Division 2

Division 3

Division 4

Division 5

Division 6

Division 7

Division 8

Division 9

Division 10

.25 (Div. 12-BC)+BC

Division 1

Kenn.+Div.17+.5(Div. 16)

+.5 (Div. 18)

Div. $19+.5($ Div. 16)+ .5 (Div. 18)

Div. 13+Div. 14+.5(0iv. 12 $-B C)$

Prosser

Divisions 2 - 10

Divisions $15 \& 20$

Div.11+.25(Div.12-BC)

Division 2

Divisions 1 \& 4

Pasco

Division 3 \& 5

Division 1

Division 2

Divisions $3 \& 4$

Divisions $5 \& 13$

Division 7

Division 8 \& 9

Division 10

Division 6 \& 11

Division 12

Division 14

Division 15 
TABLE E.1. (contd)

1970

Division 16

Division 17

Ephrata Division

KITTITAS COUNTY

Division 1

Division 2

Division 3

Division 4

Division 5

Division 6

Division 7

Division 8

Division 9

Division 10

Ellensburg Division

KLICKITAT COUNTY

Division 1

Division 2

Division 3

Division 4

Division 5

Division 6

Division 7

Division 8

Division 9

Division 10

WALLA WALLA COUNTY

Division 1

Division 2

Division 3

Division 4

Division 5

Division 6

Division 7

Division 8

Division 9

College Place

Division

Walla Walla Division

YAKIMA COUNTY

Division 1

Division 2

Division 3
1960

Division 16

Division 17

Ephrata

Division 1

Division 2

Division 3

Division 4

Division 5

Division 6

Division 7

Division 8

Division 9

Division 10

Ellensburg Division

Division 1

Division 2

Division 3

Division 4

Division 5

Division 6

Division 7

Division 8

Division 9

Division 10

Division 1

Division 2

Division 3

Division 4

Division 5

Division 6

Division 7

Division 8

Division 9

College Place

Division

Walla Walla Division

Division 1

Division 2

Division 3
$1940 \& 1950$

Division 16

Division 17

Ephrata

Division 1

Division 2

Division 3

Division 4

Division 5

Division 6

Division 7

Division 8

Division 9

Division 10

Ellensburg Division

Division 1

Division 2

Division 3

Division 4

Division 5

Division 6

Division 7

Division 8

Division 9

Division 10

Division 1

Division 2

Division 3

Division 4

Division 5

Division 6

Division 7

Division 8

Division 9

College Place

Division

Walla Walla Division

Division 1

Division 2

Division 3 
TABLE E.1. (contd)

\begin{tabular}{l} 
1970 \\
\hline Division 4 \\
Division 5 \\
Division 6 \\
Division 7 \\
Division 8 \\
Division 11 \\
Division 12 \\
Division 13 \\
Division 14 \\
Division 15 \\
Division 16 \\
Division 17 \\
Division 18 \\
Division 19 \\
Division 20 \\
Division 21 \\
Division 22 \\
Division 23 \\
Division 24 \\
Division 25 \\
Division 26 \\
Division 27 \\
Division 28 \\
Division 29 \\
Division 30 \\
Division 31 \\
Division 32 \\
Division 33 \\
Division 34 \\
Division 35 \\
Division 36 \\
Division 37 \\
Division 38 \\
Division 39 \\
Division 40 \\
Selah Division \\
Sunnyside Division \\
Toppenish Division \\
Wapato Division \\
Yakima Division \\
M0RRow CounTy \\
Boardman Division \\
Heppner Division \\
Dival
\end{tabular}

\begin{tabular}{|c|c|}
\hline 1960 & $1940 \& 1950$ \\
\hline $\begin{array}{l}\text { Division } 4 \\
\text { Division } 5 \\
\text { Division } 6 \\
\text { Division } 7 \\
\text { Division } 8 \\
\text { Division } 11 \\
\text { Division } 12 \\
\text { Division } 13 \\
\text { Division } 14 \\
\text { Division } 15 \\
\text { Division } 16 \\
\text { Division } 17 \\
\text { Division } 18 \\
\text { Division } 19 \\
\text { Division } 20 \\
\text { Division } 21 \\
\text { Division } 22 \\
\text { Division } 23 \\
\text { Division } 24 \\
\text { Division } 25 \\
\text { Division } 26 \\
\text { Division } 27 \\
\text { Division } 28 \\
\text { Division } 29 \\
\text { Division } 30 \\
\text { Division } 31 \\
\text { Division } 32 \\
\text { Division } 33 \\
\text { Division } 34 \\
\text { Division } 35 \\
\text { Division } 36 \\
\text { Division } 37 \\
\text { Division } 38 \\
\text { Division } 39 \\
\text { Division } 40 \\
\text { Selah } \\
\text { Sunnyside } \\
\text { Toppenish } \\
\text { Wapato } \\
\text { Yakima \& } 0 i v .9 \\
810\end{array}$ & $\begin{array}{l}\text { Division } 4 \\
\text { Division } 5 \\
\text { Division } 6 \\
\text { Division } 7 \\
\text { Division } 8 \\
\text { Division } 11 \\
\text { Division } 12 \\
\text { Division } 13 \\
\text { Division } 14 \\
\text { Division } 15 \\
\text { Division } 16 \\
\text { Division } 17 \\
\text { Division } 18 \\
\text { Division } 19 \\
\text { Division } 20 \\
\text { Division } 21 \\
\text { Division } 22 \\
\text { Division } 23 \\
\text { Division } 24 \\
\text { Division } 25 \\
\text { Division } 26 \\
\text { Division } 27 \\
\text { Division } 28 \\
\text { Division } 29 \\
\text { Division } 30 \\
\text { Division } 31 \\
\text { Division } 32 \\
\text { Division } 33 \\
\text { Division } 34 \\
\text { Division } 35 \\
\text { Division } 36 \\
\text { Division } 37 \\
\text { Division } 38 \\
\text { Division } 39 \\
\text { Division } 40 \\
\text { Selah } \\
\text { Sunnyside } \\
\text { Toppenish } \\
\text { Wapato } \\
\text { Yakima \& } 0 \text { iv. } \\
\text { D } 10\end{array}$ \\
\hline $\begin{array}{l}\text { Boardman \& Irrigon } \\
\text { Eightmile, Hardman, } \\
\text { N. Heppner, Pine } \\
\text { City, S. Heppner }\end{array}$ & $\begin{array}{c}\text { Boardman \& Irrigon } \\
\text { Eightmile, Hardman, } \\
\text { N. Heppner, Pine } \\
\text { City, S. Heppner }\end{array}$ \\
\hline
\end{tabular}


IABLE E.1. (contd)

\begin{tabular}{l} 
1970 \\
\hline $\begin{array}{c}\text { Ione-Lexington } \\
\text { Division }\end{array}$ \\
UMATILLA COUNTY \\
Athena Division \\
Crockett Division \\
Hermiston Division \\
Holdman Division \\
Milton-Freewater \\
Division \\
Pendleton Division \\
Pilot Rock-Echo \\
Division \\
Reservation Division \\
Stanfield Division \\
Ukiah-Meachem \\
Division \\
Umapine Division \\
Umatilla Division \\
Upper Walla Watla \\
Division \\
Weston Division
\end{tabular}

$\frac{1960}{\text { Ione-Lexington }}$

$1940 \& 1950$

Ione-Lexington

Athena Division

Crockett Division

Hermiston Division

Holdman Division

Milton-Freewater

Division

Pendleton Division

Pilot Rock-Echo

Division

Reservation Division

Stanfield Division

Ukiah-Meachem

Division

Umapine Division

Umatilla Division

Upper Walla Walla

Division

Weston Division

\author{
Precinct $10,11,13$ \\ Precinct 2,2A \\ Precinct $38,32,32 \mathrm{~A}$, \\ 33, 33A \\ Precinct $27,28,29,37,14$ \\ Precinct $4,4 \mathrm{~A}, 5,5 \mathrm{~A}$ \\ Pendleton \\ Precinct 30,35 \\ Precinct 16 \\ Precinct 31 \\ Precinct $15,36,12,39,40$ \\ Precinct 3 \\ Precinct 34,41 \\ Precinct 1, 6 \\ Precinct $7,8,9$
}




\section{APPENDIX F}

SUMMARY OF TSP COMMENTS AND BATTELLE RESPONSES 
SUMMARY OF TSP COMMENTS AND BATTELLE RESPONSES

\begin{tabular}{|c|c|c|c|c|}
\hline $\begin{array}{l}\text { Comment } \\
\text { Number }\end{array}$ & Commenter & $\begin{array}{c}\text { Page, } \\
\text { Paragraph }\end{array}$ & Comment Summary & Resolution \\
\hline 1. & J. Stohr & $\begin{array}{l}\text { Page 15, } \\
\text { para. } 1\end{array}$ & $\begin{array}{l}\text { Does a review of the school enrollment records } \\
\text { for } 1944 \text { support the assumption that the work } \\
\text { force was likely childless? }\end{array}$ & $\begin{array}{l}\text { NA - Only county-level school } \\
\text { enrollment records were used in } \\
\text { Phase I. These numbers do not } \\
\text { allow us to disaggregate the } \\
\text { fertility of construction workers. }\end{array}$ \\
\hline 2. & JS & $\begin{array}{l}\text { Page } B .2 \text {, } \\
\text { last para. }\end{array}$ & $\begin{array}{l}\text { Census estimates should be compared against } \\
\text { actual census counts to give a handle on sudden } \\
\text { population changes, as recommended by } \\
\text { Dr. Pittenger. }\end{array}$ & $\begin{array}{l}\text { This was done and summarized on } \\
\text { page } 16 \text {. Additional work may be } \\
\text { done in the future. State popula- } \\
\text { tion estimates are those produced } \\
\text { by the U.S. Census Bureau. }\end{array}$ \\
\hline 3. & JS & $\begin{array}{l}\text { Page B.2- } \\
\text { B.3 }\end{array}$ & $\begin{array}{l}\text { Have the issues of "Selection of Actual Esti- } \\
\text { mates" and "Anonymous State Population Control } \\
\text { Numbers" identified by Pittenger as highly impor- } \\
\text { tant received an adequate level of attention? If } \\
\text { not in Phase I, then when will they? }\end{array}$ & $\begin{array}{l}\text { NA - Several options for selecting } \\
\text { actual estimates were considered } \\
\text { by the TSP Demography Subcommittee } \\
\text { and were discarded. It was } \\
\text { decided that the ratio-correlation } \\
\text { method would be used. }\end{array}$ \\
\hline 4. & JS & & $\begin{array}{l}\text { The report should describe how uncertainty will } \\
\text { be estimated for the population numbers used. If } \\
\text { sensitivity analyses identify the location and } \\
\text { time of reference as key contributors to overall } \\
\text { dose estimate uncertainty, how will these census } \\
\text { predictors be used or adjusted? }\end{array}$ & $\begin{array}{l}\text { NA - At this point, uncertainty of } \\
\text { population estimates is not being } \\
\text { addressed. }\end{array}$ \\
\hline 5. & Al Conklin & & $\begin{array}{l}\text { Even though the estimates were said to include } \\
\text { age, sex, and race distributions, they are not } \\
\text { included in this report. Age is particularly } \\
\text { important, and should be included. }\end{array}$ & $\begin{array}{l}\text { See Pittenger (1991): "Letter } \\
\text { Report: Population Estimates by } \\
\text { Age, Sex and Race for } 10 \text {-County } \\
\text { Study Area." }\end{array}$ \\
\hline$N A=N_{0}$ & ction. & & & \\
\hline
\end{tabular}




\begin{tabular}{|c|c|c|c|c|}
\hline $\begin{array}{l}\text { Comment } \\
\text { Number }\end{array}$ & Commenter & $\begin{array}{c}\text { Page, } \\
\text { Paragraph }\end{array}$ & Comment Summary & Resolution \\
\hline 6. & $\begin{array}{l}\text { R.L. } \\
\text { Morri11 } \\
\text { (RLM) }\end{array}$ & & $\begin{array}{l}\text { Tackle the estimation of age-specific migration } \\
\text { for } 1944-1950 \text {, for Benton, Franklin, and Walla } \\
\text { Walla counties. }\end{array}$ & $\begin{array}{l}\text { NA - Not in current scope of work } \\
\text { authorized by the TSP. }\end{array}$ \\
\hline 7. & RLM & & $\begin{array}{l}\text { At least for the close, more affected counties, } \\
\text { age, sex, and race distributions must be specific } \\
\text { to county census division, and not rely on } \\
\text { county-wide averages. }\end{array}$ & $\begin{array}{l}\text { NA - Not in currently approved } \\
\text { scope of work. }\end{array}$ \\
\hline 8. & RLM & & $\begin{array}{l}\text { The drastic population change in Benton County } \\
\text { from } 1944 \text { to } 1945 \text { to } 1946 \text { suggests the need for a } \\
\text { finer time breakdown than yearly for Benton. }\end{array}$ & $\begin{array}{l}\text { NA - Not in currently approved } \\
\text { scope of work. }\end{array}$ \\
\hline 9. & RLM & & $\begin{array}{l}\text { Evaluate the impact of major dam construction } \\
\text { projects and the openness of the Columbia Basin } \\
\text { project on population estimates (probably just } \\
\text { for close-in counties or census units). }\end{array}$ & $\begin{array}{l}\text { NA - Not in currently approved } \\
\text { scope of work. }\end{array}$ \\
\hline 10. & $\begin{array}{l}\text { Glyn G. } \\
\text { Caldwell } \\
\text { (GGC) }\end{array}$ & $\begin{array}{l}\text { Page } \vee \\
\text { para. } 3 \\
\text { lines } 1-3\end{array}$ & $\begin{array}{l}\text { What data are missing? How much effort will be } \\
\text { needed to collect it? What are the advantages/ } \\
\text { disadvantages of this method? }\end{array}$ & $\begin{array}{l}\text { Missing data include age-specific } \\
\text { migration rates for surrogate } \\
\text { counties. Approximate estimate to } \\
\text { collect these data is } 180 \text { hours. } \\
\text { This work was proposed, but the } \\
\text { TSP decided it was not necessary. } \\
\text { The advantage of this method is } \\
\text { refined estimates resulting from } \\
\text { the inclusion of age-specifjc } \\
\text { migration data. The disadvantage } \\
\text { is the amount of time and effort }\end{array}$ \\
\hline$N A=N o$ & action. & & & \\
\hline
\end{tabular}


SUMMARY OF TSP COMMENTS AND BATTELLE RESPONSES

Document Number PNL-7263 HEDR

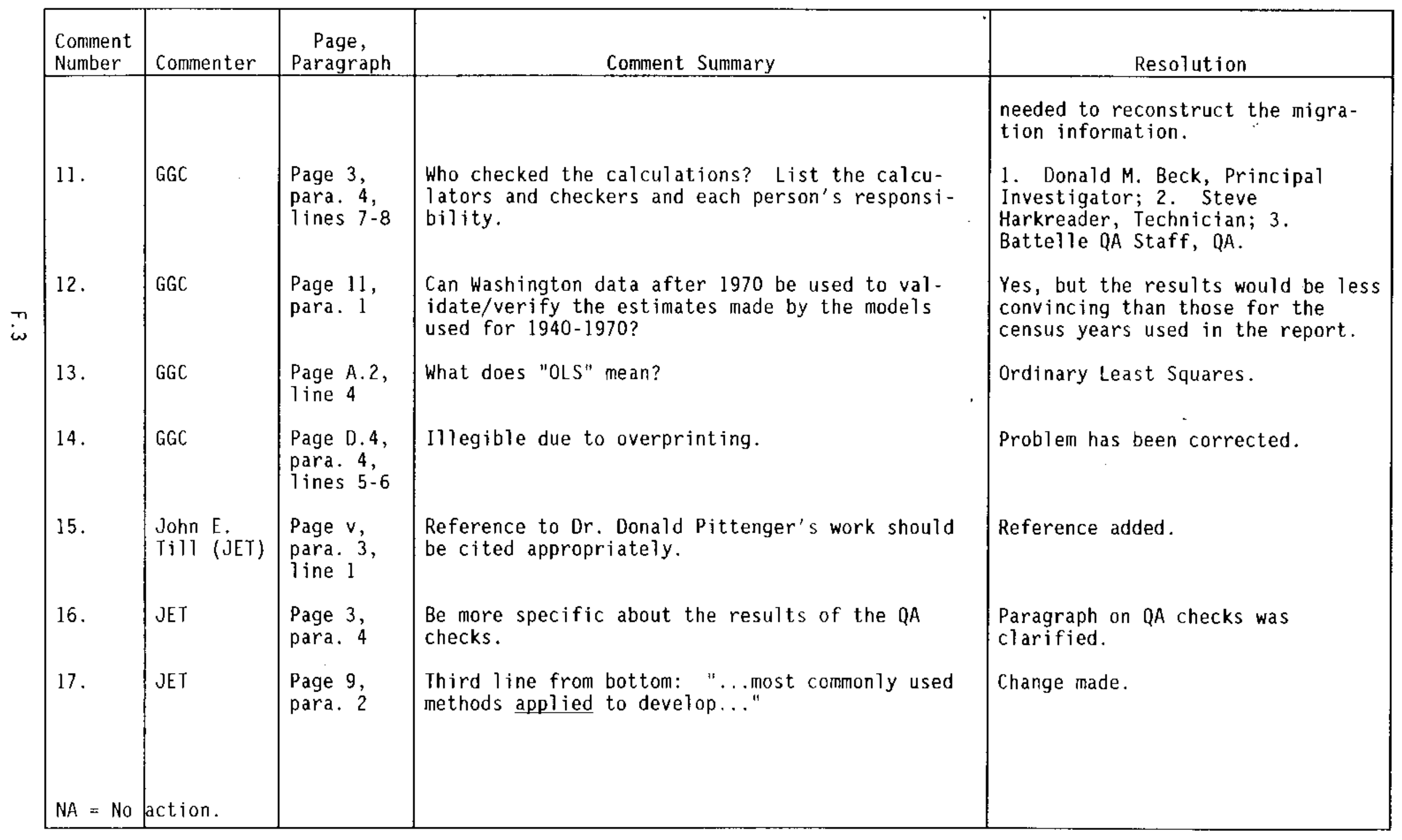


SUMMARY OF TSP COMMENTS AND BATTELLE RESPONSES

Document Number PNL-7263 HEDR

\begin{tabular}{|c|c|c|c|c|}
\hline $\begin{array}{l}\text { Comment } \\
\text { Number }\end{array}$ & Commenter & $\begin{array}{c}\text { Page, } \\
\text { Paragraph }\end{array}$ & Comment Surmary & Resolution \\
\hline 18. & JET & $\begin{array}{l}\text { Page } 10 \\
\text { eq. } 1-2\end{array}$ & $\begin{array}{l}\text { The years at the top of the ( ) to the right are } \\
\text { confusing. Also, typically equation numbers are } \\
\text { in the right margin. }\end{array}$ & $\begin{array}{l}\text { They need to be to the right of } \\
\text { the parentheses to indicate the } \\
\text { appropriate year for each } \\
\text { variable. The equation numbers } \\
\text { were moved to the right margin. }\end{array}$ \\
\hline 19. & JET & $\begin{array}{l}\text { Page } 11 \\
\text { para. } 2\end{array}$ & $\begin{array}{l}\text { Fourth line from bottom: "Deaths were not a sig- } \\
\text { nificant..." }\end{array}$ & Change made. \\
\hline 20. & JET & $\begin{array}{l}\text { Page 12, } \\
\text { Table } 1\end{array}$ & Footnotes $c$ and $d:$ "Significant to..." & Change made. \\
\hline 21. & JET & $\begin{array}{l}\text { Page 18, } \\
\text { para. } 1\end{array}$ & Would an equation not be helpful here? & $\begin{array}{l}\text { NA - Text was based on write-up of } \\
\text { contractor. }\end{array}$ \\
\hline (1) & JET & Appendix A & $\begin{array}{l}\text { Did we consider the } 3 \text { recommendations suggested } \\
\text { in Swanson's review, and what were the results? }\end{array}$ & $\begin{array}{l}\text { 1) Residual analysis completed; } \\
\text { no significant error. 2) The } \\
\text { model is conceptualized of a } \\
\text { sample of possible demographic } \\
\text { universes. The issue of } \\
\text { collinearity was not explored } \\
\text { because the model was constructed } \\
\text { only for prediction and not } \\
\text { explanation. 3) Exploring the } \\
\text { effects of changing census } \\
\text { accuracy was recommended for FY } \\
\text { l992 work but was postponed at TSP } \\
\text { direction. }\end{array}$ \\
\hline$N A=N_{0}$ & action. & & & \\
\hline
\end{tabular}

Document Title Population Estimates for Phase I 


\section{SUMMARY OF TSP COMMENTS AND BATTELLE RESPONSES}

Document Number PNL-7263 HEDR

Document Title Population Estimates for Phase I

\begin{tabular}{|c|c|c|c|c|}
\hline $\begin{array}{l}\text { Comment } \\
\text { Number }\end{array}$ & Commenter & $\begin{array}{c}\text { Page, } \\
\text { Paragraph }\end{array}$ & Comment Summary & Resolution \\
\hline 23. & $\begin{array}{l}\text { R.L. } \\
\text { Morrill }\end{array}$ & Page 4 & $\begin{array}{l}\text { Further refinement of numbers in Phase II is far } \\
\text { less important than what will be urgent: a sense } \\
\text { of the distribution of how long people lived in } \\
\text { the area (including by age). }\end{array}$ & $\begin{array}{l}\text { NA - Not in currently approved } \\
\text { scope of work. }\end{array}$ \\
\hline 24. & RLM & $\begin{array}{l}\text { Pages } 6 \\
21, \text { E.2 }\end{array}$ & $\begin{array}{l}\text { Use of age/sex and race distributions of the } \\
\text { county as a whole to determine CCD distributions } \\
\text { is (barely) acceptable for Phase I, but unaccept- } \\
\text { able for Phase II. }\end{array}$ & $\begin{array}{l}\text { NA - Not in currently approved } \\
\text { scope of work. }\end{array}$ \\
\hline 25. & RLM & $\begin{array}{l}\text { Pages } 9- \\
11,14-16\end{array}$ & $\begin{array}{l}\text { The subcommittee has already endorsed the ratio- } \\
\text { correlation methodology. }\end{array}$ & $\begin{array}{l}\text { NA - Agreed. (See response to } \\
\text { comment 3.) }\end{array}$ \\
\hline 26. & RLM & Page 17 & $\begin{array}{l}\text { Cohort-component modeling may need to be used in } \\
\text { Phase II because we need to know how many people } \\
\text { remained how long. The extrapolation methods are } \\
\text { OK for Phase I. }\end{array}$ & $\begin{array}{l}\text { NA - Not currently in approved } \\
\text { scope of work. }\end{array}$ \\
\hline 27 . & RLM & $\begin{array}{l}\text { Page } 29 \\
\text { Table } 4 \text { a }\end{array}$ & $\begin{array}{l}\text { Since the } 1944-45 \text { period is so overwhelmingly } \\
\text { important, can we, for Phase II, attempt monthly } \\
\text { estimates for Benton County? We need a sense of } \\
\text { precisely when the big changes occurred. }\end{array}$ & $\begin{array}{l}\text { NA - Not currently in approved } \\
\text { scope of work. }\end{array}$ \\
\hline 28. & RLM & Page E. 3 & $\begin{array}{l}\text { The apportioning of parts of Divisions, } 1940 \\
\text { through } 1960 \text {, needs narrative justification. } \\
\text { Suggest tables/maps of populations by } 1950 \text { divi- } \\
\text { sions (as well as 1970) for } 1950 \text {, and } 1944 \\
\text { through } 1949 \text {. }\end{array}$ & NA - Future work. \\
\hline$N A=N_{0}$ & ction. & & & \\
\hline
\end{tabular}




\section{SUMMARY OF TSP COMMENTS AND BATTELLE RESPONSES}

Document Number PNL-7263 HEDR

Document Title Population Estimates for Phase I

\begin{tabular}{|c|c|c|c|c|}
\hline $\begin{array}{l}\text { Comment } \\
\text { Number }\end{array}$ & Commenter & $\begin{array}{c}\text { Page, } \\
\text { Paragraph }\end{array}$ & Comment Summary & Resolution \\
\hline 29. & RLM & & $\begin{array}{l}\text { AD2 (Ritzville) and } 1,3,4,5,7 \text {, and } 8 \text { obviously } \\
\text { didn't grow } 1954-1958 \text {, since they didn't get } \\
\text { irrigation. The entire year-to-year growth } \\
\text { should be allocated to ADlo (0thello and vici- } \\
\text { nity)--part of the Columbia Basin Project. }\end{array}$ & $\begin{array}{l}\text { Agree in principle but need to } \\
\text { determine in future work. }\end{array}$ \\
\hline 30. & RLM & & $\begin{array}{l}\text { I would like some narrative discussion of the } \\
1944-1949 \text { breakdowns for Benton County. What are } \\
\text { the sources? }\end{array}$ & $\begin{array}{l}\text { The estimates are based solely on } \\
\text { the gross methods described in the } \\
\text { report. Refinement of these } \\
\text { methods is not in currently } \\
\text { approved scope of work. }\end{array}$ \\
\hline 31. & RLM & & $\begin{array}{l}\text { We need a little more narrative justification for } \\
\text { Franklin County, since you should have separate } \\
\text { information on the pace of opening of different } \\
\text { sections of the Columbia Basin Project. }\end{array}$ & Same response as for comment 30 . \\
\hline 32 . & RLM & & $\begin{array}{l}\text { For Grant County, } 1954-1955 \text {, the numbers are } \\
\text { clearly wrong. We need more precision for south } \\
\text { Grant County, GR 10, 12, and } 13 \text {. Again, you } \\
\text { should take advantage of Columbia Basin Project } \\
\text { data. }\end{array}$ & Same response as for comment 30 . \\
\hline 33. & RLM & Appendix A & $\begin{array}{l}\text { Did you do any residual analysis? If so, what } \\
\text { did it show? }\end{array}$ & $\begin{array}{l}\text { Yes; no significant specification } \\
\text { error. (See response to comment } \\
22 .)\end{array}$ \\
\hline 34. & RLM & Page B. 2 & $\begin{array}{l}\text { Identify when the major construction projects } \\
\text { occurred and where; i.e., dams on the Columbia } \\
\text { River, the Columbia Basin Project. }\end{array}$ & NA - Future work. \\
\hline
\end{tabular}


SUMMARY OF TSP COMMENTS AND BATTELLE RESPONSES

Document Number PNL-7263 HEDR

Document Title Population Estimates for Phase I

\begin{tabular}{|c|c|c|c|c|}
\hline $\begin{array}{l}\text { Comment } \\
\text { Number }\end{array}$ & Commenter & $\begin{array}{l}\text { Page, } \\
\text { Paragraph }\end{array}$ & Comment Summary & Resolution \\
\hline 35 . & RLM & Page E.l & $\begin{array}{l}\text { The simple interpolation method is usualiy ok, } \\
\text { but not for areas affected by episodic construc- } \\
\text { tion and land opening. Phase II will need some } \\
\text { refinement here. }\end{array}$ & Agree. \\
\hline 36 . & RLM & & $\begin{array}{l}\text { Some close-in but large census sub-divisions } \\
\text { should be divided, while some far-away divisions } \\
\text { can be combined. }\end{array}$ & $\begin{array}{l}\text { NA - Not in currently approved } \\
\text { scope of work. }\end{array}$ \\
\hline 37. & RLM & & $\begin{array}{l}\text { The study area should probably be extended some- } \\
\text { what to the east and perhaps to the south for } \\
\text { Phase III, but counties could be the units of } \\
\text { analysis. }\end{array}$ & TSP decision. \\
\hline 38. & $\begin{array}{l}\text { M.A. } \\
\text { Robkin } \\
\text { (MAR) }\end{array}$ & Page 18 & $\begin{array}{l}\text { Cohort Interpolation: The number of males of age } \\
46 \text { in } 1960 \text { are divided by the number at age } 36 \text { in } \\
1950 \text { in Grant county. This is not logical and } \\
\text { one of these ages should be corrected. }\end{array}$ & $\begin{array}{l}\text { NA - This ratio is appropriate for } \\
\text { determining change from } 1950 \text { to } \\
1960 \text { for this age category. }\end{array}$ \\
\hline 39. & MAR & Page 19 & $\begin{array}{l}\text { Top of page: The racial categories are white, } \\
\text { non-white, negroes/blacks, and Indians. This is } \\
\text { a bizarre categorization. }\end{array}$ & $\begin{array}{l}\text { The categories are provided by the } \\
\text { U.S. Census Bureau. }\end{array}$ \\
\hline 40. & MAR & Page B. 4 & $\begin{array}{l}\text { In the expression for RATEYRn: as written the } \\
\text { value is always negative. Should the "minus } \\
\text { sign" be a "plus sign"? }\end{array}$ & Equation was revised. \\
\hline 41. & MAR & Page C.1 & $\begin{array}{l}\text { In the section on step } 1 \text { : There are two typos. } \\
\text { In the ratio itself, the lower date should be } \\
1940 \text {. In the line below, "bi" should be "Bi". }\end{array}$ & Changes made. \\
\hline$N A=N_{0}$ & action. & & & \\
\hline
\end{tabular}


SUMMARY OF TSP COMMENTS AND BATTELLE RESPONSES

Document Number PNL-7263 HEDR

Document Title Population Estimates for Phase I

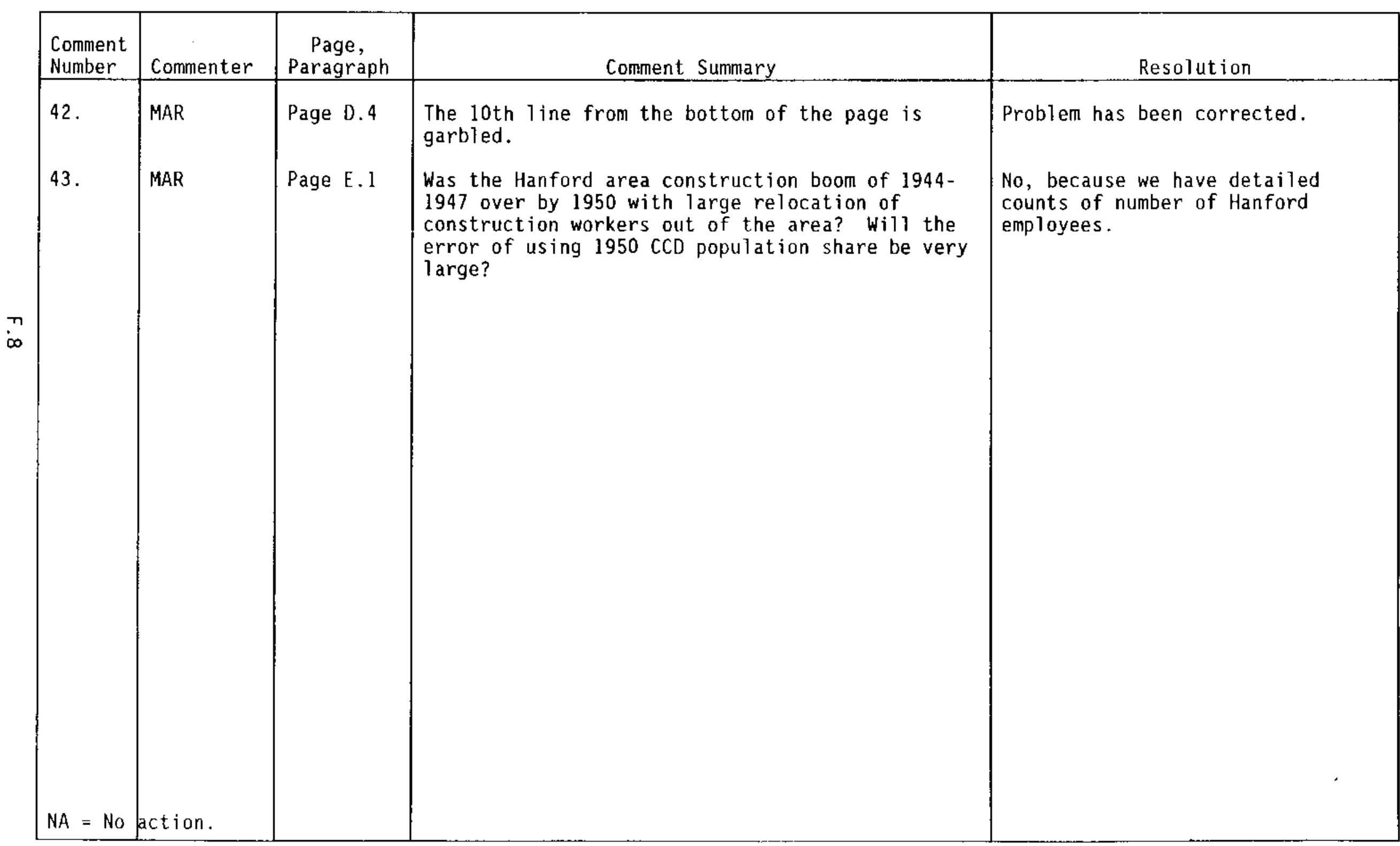




\section{DISTRI8UTION}

No. of

Copies

\section{OFFSITE}

18 Technical Steering Panel

D. S. Barth

University of Nevada

4505 Maryl and Parkway

Las Vegas, NV 89154

W. A. Bishop

2503 Wedgewood Court SE

01ympia, WA 98501

M. L. Blazek

Oregon Department of Energy

625 Marion Street N.E.

Salem, OR 97310

G. G. Caldwell

Director

Tulsa City-County Health Dept

4616 East 15th Street

Tulsa, OK 74112

S. N. Davis

Dept. of Hydrology \& Water Resources

B1dg. 11

University of Arizona

Tucson, AZ 85721

N. J. Germond

224 Iron Mountain Blvd.

Lake Ozwego, OR 97034

P. C. Klingeman

Civil Engineering Dept.

Oregon State University

Appejin $\mathrm{Hall} 202$

Corval1is, OR 97331-2302
No. of

Copies

K. J. Kopecky

Fred Hutchinson Cancer

Research Center

1124 Columbia Street

Seattle, WA 98104

P. D. McGavran

Dept. of Health and Welfare

450 W. State Street, 4th Floor

Boise, ID 83720-5450

R. L. Morrill

Dept. of Geography

Dartmouth College

Hanover, NH 03755

A. H. Murphy

Dept. of Atmospheric Sciences

Oregon State University

Corvallis, OR 97331-2209

D. W. Price

Agricultural Economics

Hulbert Hall Room 2lI

Washington State University

Pullman, WA 99164-6210

M. A. Robkin

Radiological Sciences SB-75

University of Washington

Seattle, WA 98195

G. S. Roessler

202 Nuclear Sciences Center

University of Florida

Gainsville, FL 32611

B. Shleien

2421 Homestead Drive

Silver Springs, MD 20902 
No. of

Copies

A. P. Slickpoo, Sr.

P. 0. Box 331

809 Nez Perce Lane

Kamiah, I0 83536

J. E. Ti11

Rt. 2 Box 122

Neeses, SC 29107

D. E. Walker, Jr.

P.0. Box 4147

Boutder, CO 80306

other

2 DOE Office of Scientific and Technical Information

Technical Information Center

P.0. Box 62

Oak Ridge, TN 37830

B. G. 8rooks, EH-42l

Department of Energy

Room J-112

Germantown, MD 20545

12 K. Charlee

Office of Nuclear Waste Mgmt.

Department of Ecology

99 South Sound Center

Mail Stop PV-11

OTympia, WA 98504

6 W. A. Glass

13874 E. 47th Lane

Yuna, AZ 85365

H. Sage

Centers for Disease Control

1600 Clifton Road

Atianta, GA 30333
No. of

Copies

K. F. Sparks

Shea \& Gardner

1800 Massachusetts Ave., N.W.

Washington, DC 20036

J. Thomas

HEAL

1720 N. Ash

Spokane, WA 99205

\section{ONSITE}

2 DOE Field office, Richland
R. F. Brich, TSD
A5-55
M. W. Tiernan, TSD
$A 5-55$

35 Pacific Northwest Laboratory

D. M. Beck (5)

K6-57

J. M. Daer (5)

$K 6-89$

R. L. Dirkes

$\mathrm{K} 6-13$

M. D. Freshley

$\mathrm{K} 6-77$

R. 0. Gilbert

$\mathrm{K} 7-34$

G. L. Harvey

$\mathrm{K} 1-36$

C. M. Heeb

$\mathrm{K} 6-42$

T. A. Ikenberry K3-54

A. H. McMakin K6-86

B. A. Napier

$\mathrm{K} 3-54$

R. E. Rhoads

$\mathrm{K} 6-64$

H. L. Templeton

$\mathrm{K} 1-30$

H. H. Walters

K6-09

G. L. Wilfert (5)

K6-54

Public Reading Room (5) Al-65

Pubtishing Coordination $\mathrm{Kl}-06$

Records Center

$\mathrm{K} 3-70$

Technical Library

P8-55 